$$
\begin{aligned}
& \text { DCE/TR/R72S. TI } \\
& \text { DOE/ER/13735--TI } \\
& \text { DE93 } 001704
\end{aligned}
$$

FINAL REPORT

\title{
SELF SHIELDING OF SURFACES IRRADIATED BY INTENSE ENERGY FLUXES
}

\author{
P.L. Varghese, J.R. Howell, and Alan Propp
}

The University of Texas at Austin

\author{
PREPARED FOR THE U.S. DEPARTMENT OF ENERGY \\ UNDER \\ GRANT DE-FG05-87ER13735
}

AUGUST 1, 1991 


\section{TABLE OF CONTENTS}

SECTION

PAGE NUMBER

INTRODUCTORY REMARKS ............................................................... ii

CHAPTER 1: INTRODUCTION ............................................................ 1

CHAPTER 2: EXPERIMENTAL FACILITY ....................................... 9

CHAPTER 3: RESULTS AND DISCUSSION OF EXPERIMENT 1 ..... 34

CHAPTER 4: TARGET POSITION MEASUREMENTS .......................... 62

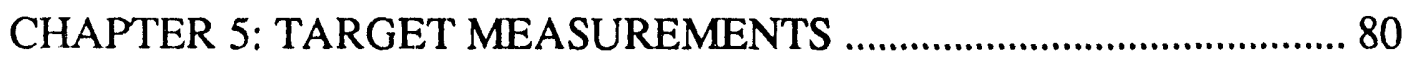

CHAPTER 6: SUMMARY AND CONCLUSIONS ................................... 95

APPENDIX I: EQUIPMENT SPECIFICATIONS ................................. 99

APPENDIX II: DETAILS OF ELECTRON DENSITY CALCULATIONS

BY THE GRAPHICAL METHOD ....................................108

APPENDIX III: EFFECT OF BOUNDARY LAYERS ON TEMPERATURE DETERMINATION VIA BOLTZMANN PLOTS .......... 138

APPENDIX IV: ERROR ESTIMATES FOR IN-SITU TEMPERATURE

CALCULATIONS ....................................................................... 141

APPENDIX V: FEASIBILITY ANALYSIS OF TWO-PHOTON, LASER INDUCED FLUORESCENCE STUDIES OF RAIL-GUN

PRODUCED HYDROGEN PLASMAS .............................. 146

BIBLIOGRAPHY:

158

APPENDIX VI: COPIES OF RECENT PAPERS RESULTING FROM

THIS WORK 


\section{INTRODUCTORY REMARKS}

This report details the work carried out in completion of DOE Grant DE-FG05-87ER 13735. In addition to the research reported here, the grant resulted in dissertations completed by Dr. Y-K Chen (Self Shielding of Surfaces Irradiated by Plasma Fluxes) and Dr. Alan Propp ( Experimental Measurement of Transient Plasma Surface Interactions ), and an MS thesis completed by Mr. Brian Harden (Experimental Investigation of a Hydrogen Plasma Railgun). Much of the material in this final report is excerpted from Dr. Propp's dissertation.

Two technical papers have been accepted for IEEE Transactions that directly report on the this research, and are appended to this report as Appendix 6. Earlier papers were included in annual reports. In addition, novel analytical methods developed to support this work have led to a number of publications, some detailed in earlier work. The spin-off from this work has led to novel methods for treating the radiative transfer equation and the Boltzmann equation. This work was inspired by the present contract research, although it was supported by other funds. 


\section{CHAPTER 1}

\section{INTRODUCTION}

\subsection{Railgun fundamentals}

A railgun consists of two conductors (rails) separated by electrical insulating sidewalls so as to leave a gap. See Fig. 1.1 for a schematic diagram of the railgun geometry. A conducting armature is placed in the gap between the rails. When a voltage is applied to the rails, via some external source (e.g., a capacitor bank), current flows through the rail at positive potential, through the conducting armature, and back through the rail at negative potential. The driving force behind the armature in a railgun is the electromagnetic force which is created when current flows through the armature orthogonal to the magnetic field set up by the current flowing through the rails (see Fig. 1.1). The differential force on the armature can be expressed as

$$
\mathrm{d} \mathbf{F}=\mathbf{J} \times \mathbf{B} \mathrm{dV}
$$

where $\mathbf{J}$ is the current density $\left(A / \mathrm{m}^{2}\right), \mathbf{B}$ is the magnetic field (T), and $d V$ is the differential volume element .1 .1

The armature in a railgun can be any conductor, although solid and plasma armatures are most common. The majority of solid armature railguns have a nonconducting projectile placed ahead of the armature. The magnetic force applied to the armature is then transmitted to the solid projectile via direct contact, accelerating it 
Figure 1.1 Railgun geometry

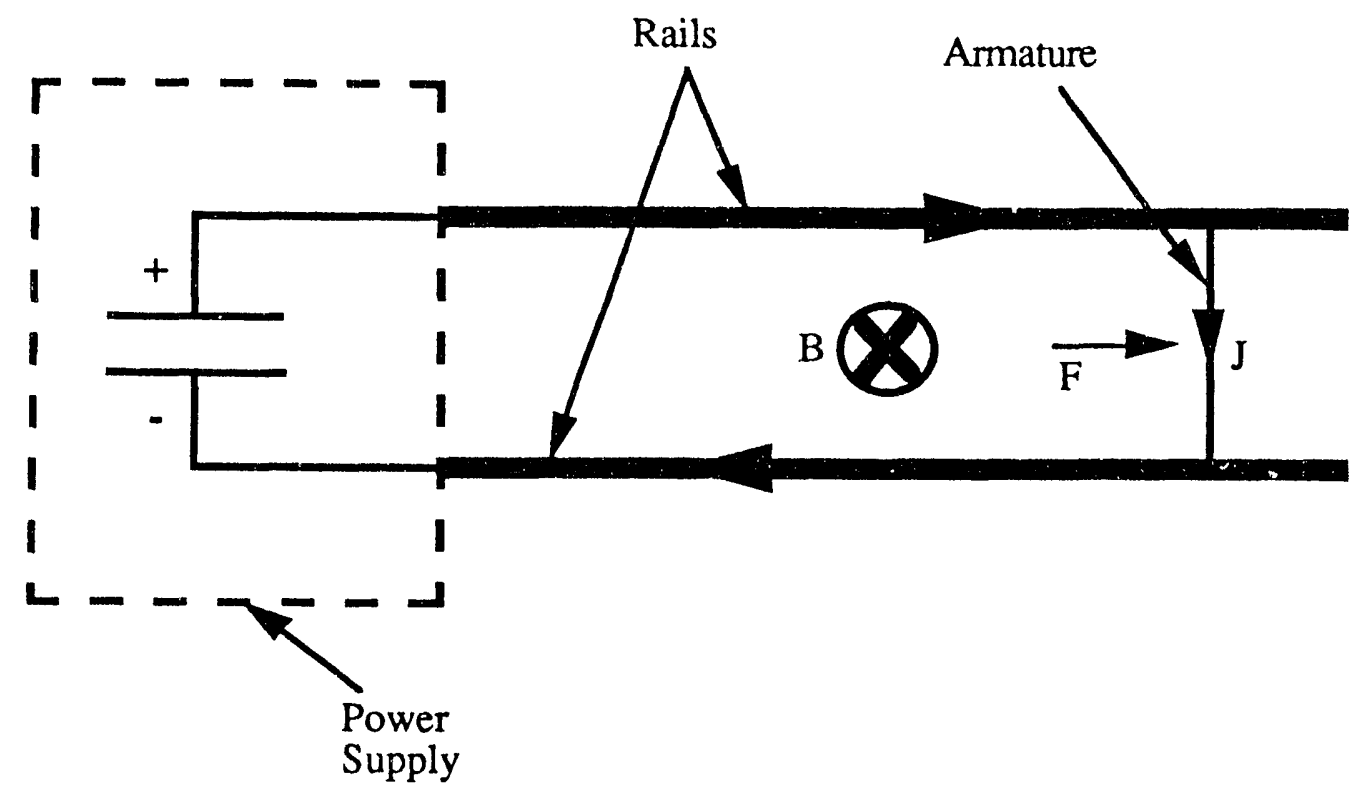

down the barrel of the railgun. Solid projectile railguns are of military interest because they can accelerate particles to much higher speeds than can conventional chemical guns.

There are two primary configurations for plasma armature railguns. One employs a fuse which generates the plasma prior to its injection into the railgun. The railgun is fired with this plasma already formed and in its breech. The other design uses a device to inject a gas between the rails of the gun. This gas is then ionized by a high voltage applied between the rails. Plasma armature railguns can also be used to accelerate a solid projectile, but are frequently used solely for the purpoie of plasma generation. It is this type of railgun which is the focus of this study.

\subsection{Motivation for this research}


For several decades railguns have been studied as a means to accelerate particles to high velocities. The goals of these studies have been, and continue to be, quite diverse. The initial motivation for this particular research came from an interest in the ablation caused by plasma impact on the first-wall of a fusion confinement vessel. It is thought that a phenomenon known as self-shielding affects the amount of wall ablation caused by plasma impact. Self-shielding results when wall material is ablated during the initial plasma impact, and forms a shield against later plasma bombardment. Characterization of the plasma-wall interaction is a difficult engineering problem because of the variety of ways in which energy is transferred from the plasma to the wall. Plasma particle impact causes erosion and heating, electromagnetic radiation from the plasma produces heating, and neutrons cause radiation darnage, heating, and can result in the formation of highly radioactive materials. 1.2

In addition to first-wall studies, other possible applications of the study of plasma-surface interactions include wear induced on the tips of tungsten-inert gas welding electrodes due to plasma ablation, ablation of surfaces of re-entry vehicles at high altitudes, wear on the inner walls of spacecraft propulsion systems, and material deposition onto substrates.

\subsection{Applications of plasma armature railguns in research}

Currently, plasma acceleration by railguns has several important applications. One of these is the deposition of matter onto a substrate which has been impacted by a railgun accelerated plasma. ${ }^{1.3}$ This process can be used to build up thick arnorphous deposits on transformer cores or for the deposition of stainless steel on 
mild steel substrates to improve their corrosion resistance. ${ }^{1.4}$ Another possible application of railguns is to study the effects of high temperature plasma on surfaces. These surfaces may include rocket engine walls, magnetic confinement vessels, and the surfaces of space re-entry vehicles. Because of the high speed to which railguns are able to accelerate plasma, railgun technology may be useful to simulate the conditions occurring in these environments. Plasma armature railguns are also being used to accelerate solid projectiles for military applications.

A future application of railgun technology may be the propulsion of space vehicles. One important parameter in evaluating the performance of a rocket is the speed at which the propellent is ejected, as this speed is a critical factor in the determination of the vehicle's maximum velocity. ${ }^{1.5}$ It has been calculated that the exhaust velocity of a practical interplanetary engine should be about $3.0 \times 10^{3}$ $\mathrm{km} / \mathrm{sec} .^{1.6}$ Using a momentum transfer setup, Marshall determined that his first railgun accelerated plasma to over $2.5 \times 10^{3} \mathrm{~km} / \mathrm{s}$, which made it a very interesting prospect for this application. More recent experimental studies have found plasma velocities in the 10 to $50 \mathrm{~km} / \mathrm{s}$ range. Because of these low plasma ejection velocities and because of the low power output-to-weight ratio of railguns, railguns have been infeasible for space propulsion up to this time. However, as more sophisticated railgun designs are developed and as smaller, more powerful pulsed-power sources become available, railgun technology may again become attractive for space vehicle propulsion.

\subsection{Recent research topics}


One area of interest to several groups of experimenters is the ablation of rails by the plasma armature. In addition to the obviously detrimental effects of rail wear such as maintenance costs, rail ablation has been found to be one of the major limiting factors of the speed to which a solid projectile can be accelerated. Aigner and Igenbergs 1.7 found that in addition to mechanical friction between the projectile and the wall and the viscous drag of the wall on the plasma, the mass of the electrodes ablated into the plasma arc must be included to accurately model the movement of a projectile in a railgun. They found that of the $28 \%$ reduction in velocity from the "ideal", $15 \%$ was due to rail ablation effects, $9 \%$ was due to mechanical friction, and $4 \%$ was due to viscous arc drag.

Other investigators have been studying ways in which to reduce the drag on plasma armatures, thereby increasing their speed. Parker ${ }^{1.8}$ used a method he called a "segmented rail surface" in his railgun construction. The purpose of this design was to reduce the amount of "restrike" which occurs in the conventional railgun design. Restrike occurs when an isolated plasma develops following the main plasma arc, reducing the total force on the main plasma. In this design, restrike was eliminated by progressively disconnecting segments of the rail surface after the plasma armature had passed. Parker found that this design allowed for a $32 \%$ increase in plasma velocity for the entire length of the barrel, with a final velocity of $16 \mathrm{~km} / \mathrm{s}$.

Two factors limiting the maximum armature velocity achievable in railguns are the impedance of the rails and the frictional drag on the projectile. Holland 1.9 designed and tested what he called a "distributed energy store (DES) railgun" to try to overcome the rail impedance problem. The DES design employs multiple current sources connected to distributed points along the bore of the railgun. These current 
sources are activated sequentially as the armature moves along the bore such that the current is fed to the railgun from immediately behind the armature, thereby reducing the length of the rails through which the current must flow. During the process of comparison of his experimental results with those predicted using a model which ignored frictional drag Holland found that frictional drag was significant. He analyzed the mechanism and approximate magnitude of the frictional drag on the projectile.

The exact cause of rail ablation has been the topic of investigation of several groups. Derbidge and Powars 1.10 found that, for copper rails, the main ablation mechanism is boiling and vaporization. They found that the material melted during plasma passage, prior to boiling, is not swept away but remains attached to the surface, and that the ablated material leaves the railgun primarily as unionized vapor. To test their theoretical models they used a $0.6 \mathrm{~m}$ long, $1 \mathrm{~cm}$ square bore gun with copper rails. By extrapolating their experimental mass loss results, they predicted that no ablation of the rails would occur for arc energies less than about $10 \mathrm{~kJ}$ because their would be insufficient energy to heat the rails to their boiling temperature.

Jamison and Burden ${ }^{1.11}$ recently conducted an extensive diagnosis of the plasma arc in a plasma armature driven, solid projectile railgun. One of their findings was that the armature lerigth decreased roughly linearly with increasing current, from about $32 \mathrm{~cm}$ at $0.8 \mathrm{MA}$ to about $27 \mathrm{~cm}$ at $1 \mathrm{MA}$. They also found that the luminous front lagged behind the current carrying portion of the armature. The reason given for this phenomenon was that the luminous portion of the plasma seeped past the projectile as it progressed down the bore of the railgun. 


\subsection{Characterization of plasma armatures}

Accurate measurement of parameters such as temperature, velocity, and composition of railgun generated plasma armatures is of interest for several reasons. We were interested in these parameters because they were initial conditions for studies of the damage caused to targets by the impingement of the plasma. Similarly, experimentalists interested in the deposition of plasma onto a substrate could use these quantities to help characterize the deposition process. ${ }^{1.12}$ Also, researchers accelerating solid projectiles with plasma armatures could use temperature and density measurements to help determine the mechanisms causing ablation of both the projectile and the rails of the gun. 1.13

Several methods for determining plasma armature characteristics in railguns have been presented in the literature. Ray 1.14 used an iterative technique in which, for an assumed plasma mass, he varied the temperature until the calculated voltage drop across the plasma armature agreed with the measured value. In this way he was able to predict as functions of mass the following parameters of the plasma arnature in his gun: 1) degree of ionization, 2) density and electron density, 3) collision frequency, 4) conductivity, 5) length, and 6) resistance. Marshall 1.15 has calculated the temperature of the plasma via measurement of the velocity of the shock wave generated when the solid projectile leaves the railgun muzzle. He also outlined a method of temperature measurement in which holes normal to the railgun bore were machined in the rails. ${ }^{1.16}$ These holes acted as plasma jets. By measuring the velocity of the plasma issuing from these jets he calculated the local plasma temperature as the plasma pulse passed a given hole. Sedghinasab et al. ${ }^{1.17}$ have described a method in which an assumed teniperature profile was used to predict the absorption profile for 
aluminum and copper lines in the $290-350 \mathrm{~nm}$ spectral region. This predicted absorption profile was compared with the measured absorption profile and the assumed temperature profile was modified until the predicted and measured absorption profiles were in agreement. Kiwamoto 1.18 has estimated the electron temperature of the plasma armature using the ratio of intensities of two barium spectral lines. The barium lines were the result of erosion and subsequent ionization of a barium electrode.

\subsection{Conclusions}

The above outlined methods of plasma armature characterization have been tailored to very specific experimental situations. In addition, several of the experimenters have used approaches in which parameters other than those being sought were measured and then the desired parameter deduced from these results. Temperature measurement in particular has been by means which would seem to be of limited applicability to most railgun systems. This dissertation will outline a direct methods of temperature, density, composition, and velocity measurement which should be widely applicable to railgun systems. The measurements demonstrated here should prove useful to other experimenters trying to accurately characterize the plasmas being emitted by their railguns, and should provide a useful basis for further studies of plasma/target interaction. 


\section{CHAPTER 2}

\section{EXPERIMENTAL FACILITY}

\subsection{Vacuum Chamber System}

The vacuum chamber was built by Austin Scientific Inc. It was constructed of 16 inch diameter stainless steel tube with $6.35 \mathrm{~mm}(1 / 4 \mathrm{inch})$ thick sidewalls (see Figs. $2.1,2.2$, and 2.3 for views of the vacuum chamber system). Thirteen $70 \mathrm{~mm}$ (2.75 inch) diameter flanges were installed on the chamber to allow for flexibility in the placement of the various feedthroughs to be described below. These flanges were fitted with copper gaskets, which allow for a much better seal than rubber ones of this size. Four of these flanges were fitted with optical viewports to allow for observation of the inside of the vacuum chamber. One flange was fitted with an eight-pin electrical feedthrough which is used to transmit the data from the magnetic pick-up coils ( $(\dot{B}$-probes) (see Section 3.1). Another was fitted with a sixteen pin electrical feedthrough, four of which are currently used for $\stackrel{\circ}{\mathrm{B}}$-probes, and the rest of which are unused. A feedthrough with six optical fibers was installed on one of the flanges on top of the chamber.

Extensive safety precautions were taken in feeding hydrogen into the gas injection valves (see section 2.2) through one of the $70 \mathrm{~mm}$ flanges. Figure 2.4 is a schematic diagram of the hydrogen feedthrough system. Valves 1, 2, and 3 were used to allow hydrogen to flow through various stages of the system. They were 
Figure 2.1 Vacuum Chamber End View

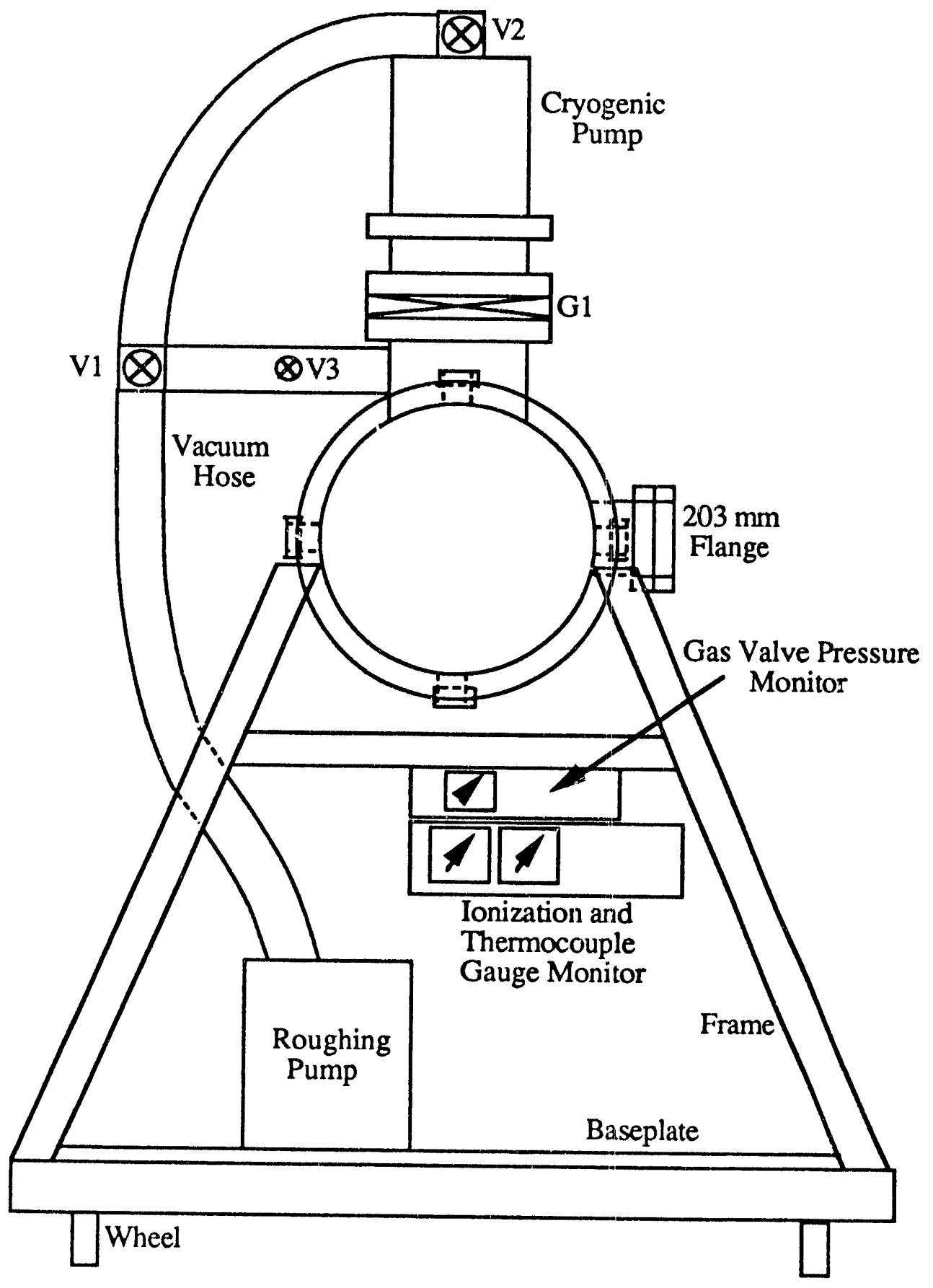


Figure 2.2 Vacuum Chamber Top View

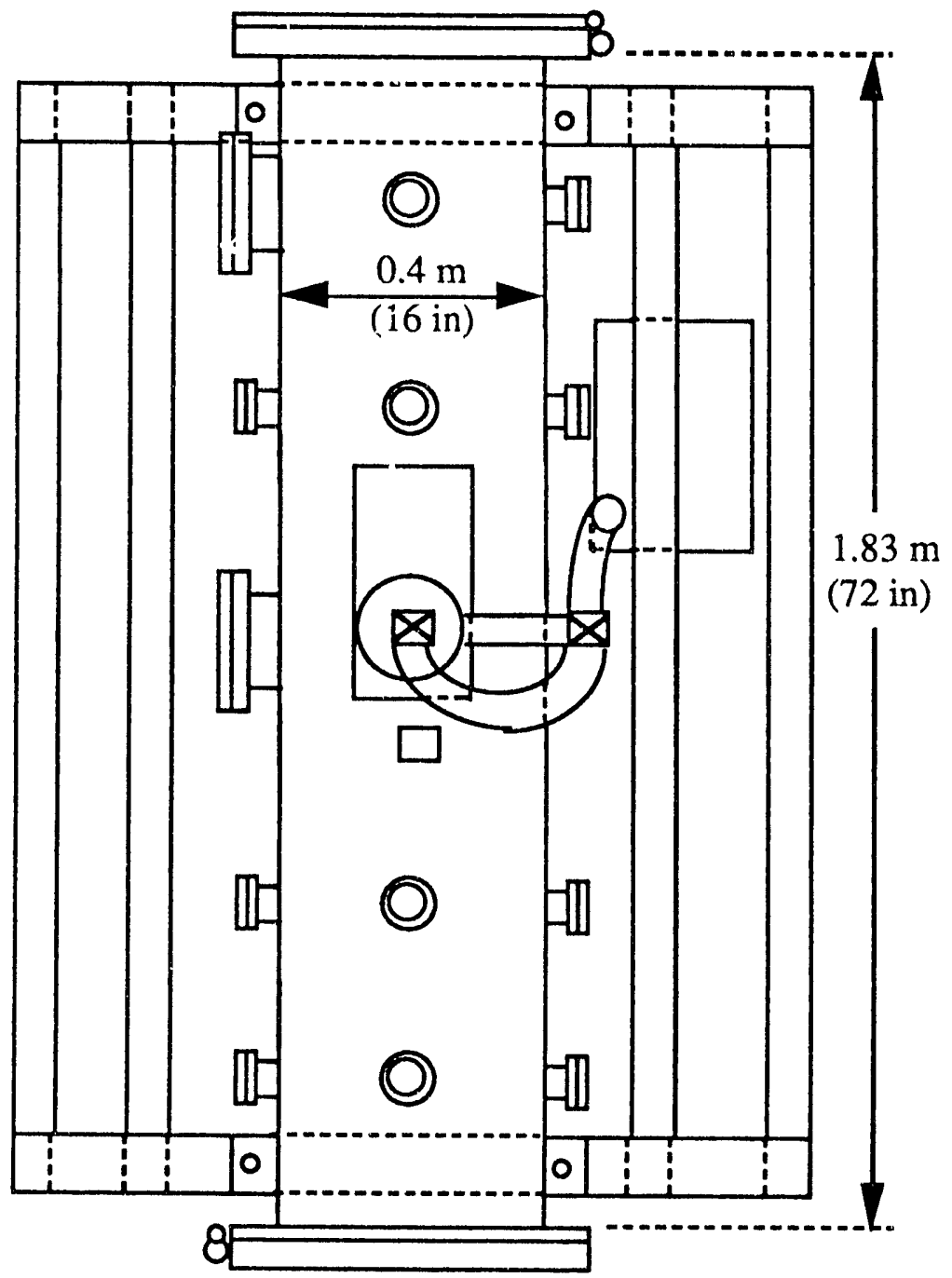

opened successively to pressurize the $\mathrm{H}_{2}$ injection valve, and all were shut prior to firing of the gun. Valve 4 was opened only when valve 3 was shut, allowing the $\mathrm{H}_{2}$ 
Figure 2.3 Vacuum Chamber Side View

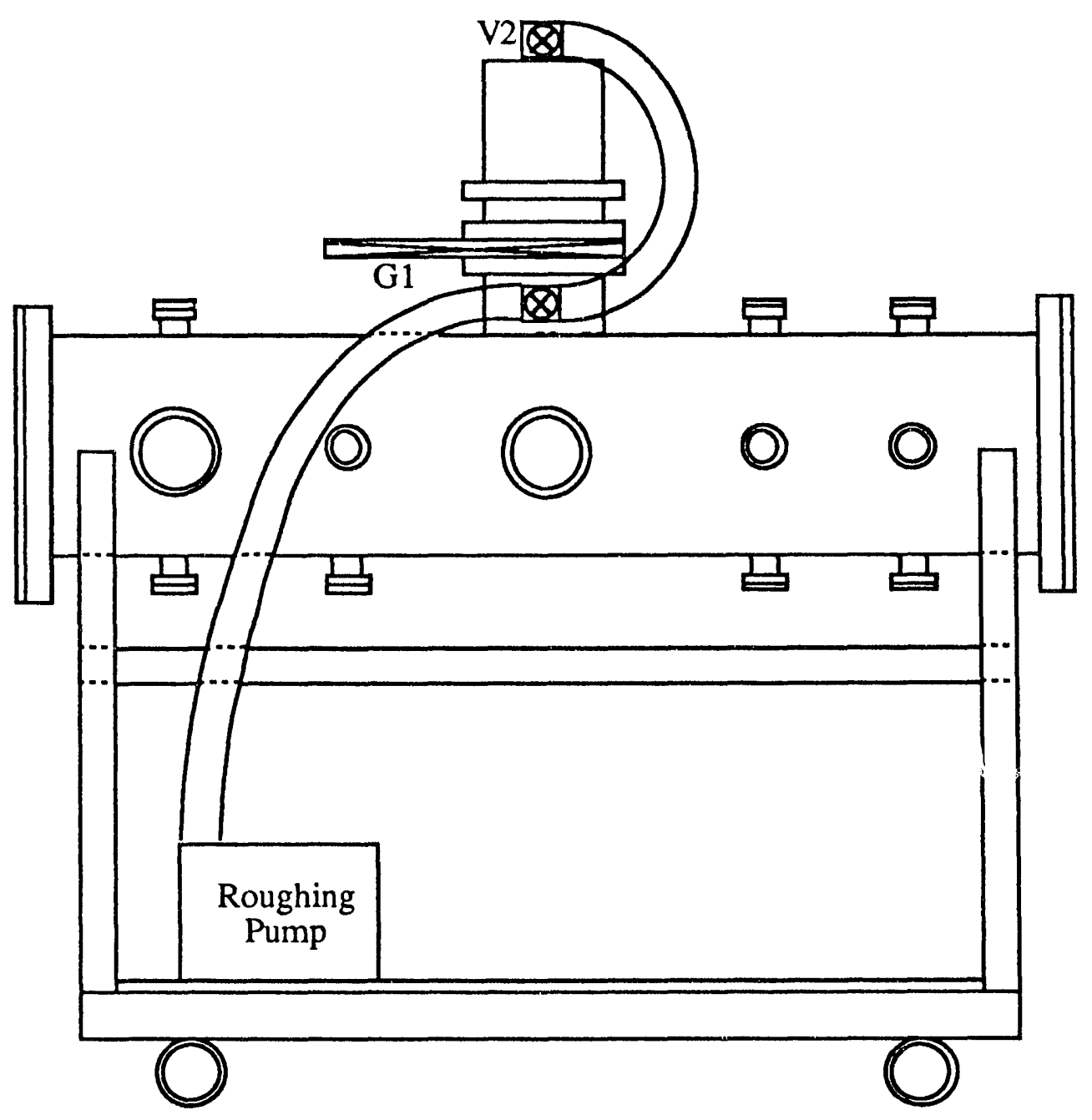

injection valve to be evacuated by the pumps after it had been cleaned or adjusted. Using valve 4 and the pressure gauge shown, the back pressure to the $\mathrm{H}_{2}$ injection valves could be accurately adjusted. 
Figure 2.4 Hydrogen Supply System to Piezoelectric Valve

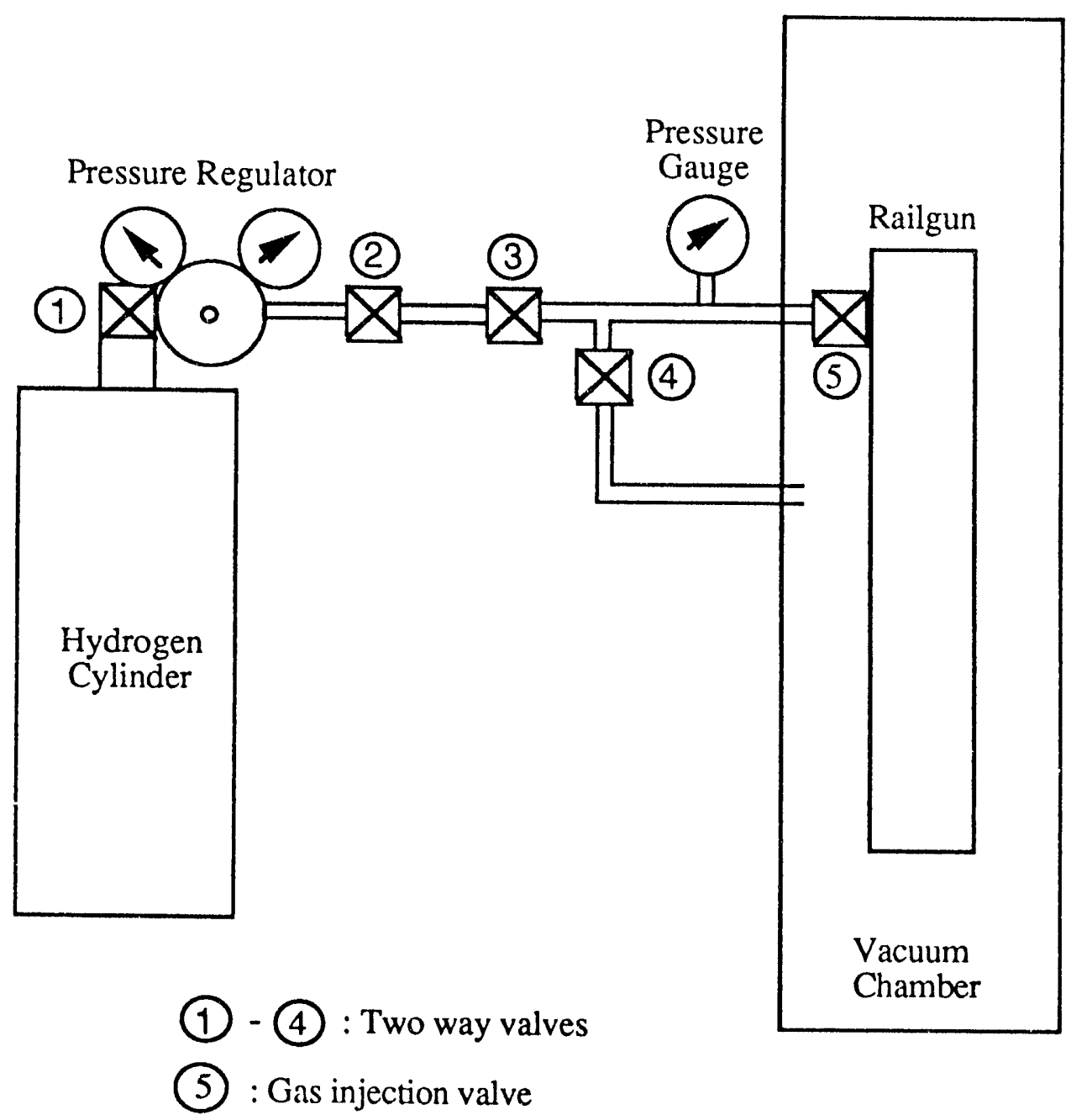

Two $152 \mathrm{~mm}(6 \mathrm{inch})$ diameter flanges were also installed on the chamber. One of these was located near the end of the chamber and was fitted with a coaxial power feedthrough from the capacitor bank to the railgun. The other large flange was 
installed in the middle of the chamber to allow access to the middle portion of the railgun. One end of the chamber was fitted with a hinged flange for ease of access to the railgun. A hinge was subsequently designed and installed on the other end flange as it was realized that access to the target without removing the entire gun would be useful. An infrared viewport was also installed on the end flange nearest the railgun muzzle to allow for temperature measurement of targets placed downstream of the railgun muzzle. The $152 \mathrm{~mm}$ flanges and the er. $\mathrm{l}$ flanges were fitted with synthetic rubber (Buna N) O-rings because these flanges are opened more frequently than the small ones and copper gaskets for flanges of these sizes are very expensive.

Two pumps were installed on the chanber; a roughing pump and a cryogenic (cryo) pump. Three valves were used to control the sections of the vacuum system that were being evacuated (see Fig. 2.1). Valve V1 allowed the roughing pump to, pump on the main chamber and the cryo-pump. Valve V2 was closed when it was necessary to use the roughing pump on the main chamber only.. The third valve is the large gate valve G1 connecting the cryo-pump to the chamber. Valve V3 was used to vent the outside pressure to the chamber so it could be opened.

The roughing pump served two purposes. Firstly, it was used to pump the cryo-pump down to a pressure at which it can be started ( $\approx 3 \mathrm{mTorr})$. Secondly, it was used to pump the vacuum chamber down to a level at which the cryo-pump could be applied. This process took approximately one hour. After the cryo-pump was evacuated using the roughing pump and turned on, it took approximately $2^{1 / 2}$ hours to cool down to its operating temperature $(\sim 14 \mathrm{~K})$. With the cryo-pump cooled and the vacuum chamber evacuated to about 3 mTorr with the roughing pump, the cryo-pump was opened to the main chamber. When this is done the vacuum chamber 
pressure decreased from $\sim 3 \mathrm{mT}$ Torr to $\sim 5 \mu$ Torr in a matter of minutes. We were abie to maintain a vacuum chamber pressure of about $1 \mu$ Torr.

The entire vacuum chamber system is mounted on a portable frame. A $12.7 \mathrm{~mm}(1 / 2$-inch) steel baseplate was installed on this frame to allow for portable mounting of the pumps and other equipment. However, it was found that the roughing pump vibrated excessively so it was later placed on the floor.

\subsection{Railgun System}

A primary consideration in the design of the railgun was the desire to study the plasma optically prior to its exit from the railgun. We felt that determination of the temperature and density distributions of the plasma armature within the gun would give insight into the mechanisms which govern its formation and propagation. For this reason a parallel-plate design with optically transmitting sidewalls was chosen over the coaxial one. With optically-transmitting, insulating sidewalls, observation of the plasma armature along the entire railgun length was possible, except where the view was obstructed by the support structure.

The size and length of the railgun were chosen rather arbitrarily. Plasma railguns reported in the literature vary in length from several centimeters to over a meter. One meter was chosen as the length for the railgun, as that would allow ample length for observation of the evolution of the plasma armature. This length was also convenient for installation in an existing vacuum chamber at the Center for Electromechanics (CEM). The length was later reduced to $85.5 \mathrm{~cm}$ to help prevent the striking of an arc between the end of the rails and the support structure. The railgun has a $2.5 \mathrm{~cm}$ by $7.5 \mathrm{~cm}$ rectangular bore with rails made of high purity 
copper. The location of the initiation of the plasma armature was controlled by attaching a copper nipple to each of the rails near the breech. We initially chose fused quartz as the miterial for the insulating sidewalls of the gun. In addition to its ability to transmit in the ultra-violet, Petersen et $a^{2.1}$ found that fused quartz showed no effects of ablation in railgun tests similar to ours. However, we found that the sidewalls became coated with plasma deposits after several firings of the railgun, so we have now switched to ordinary window glass as the insulating material. The fact that the glass ablates actually helped us in the determination of the plasma temperature (see Section 4.1). The glass insulating sidewalls are very inexpensive compared to the quartz ones, and can therefore be replaced as needed rather than cleaned. We have found that we are able to evaluate the plasma electron temperature and density without using the ultraviolet transmissivity of quartz, so this change in sidewall material has not been a hindrance. Figure 2.5 is a schematic diagram of the railgun assembly.

A stainless steel support structure constrained the railgun from moving or flexing. This support structure was desigived by B. Harden after an analysis of the forces involved. Optical rails were attached to the support structure parallel to the axis of the railgun. Diagnostic equipment could be accurately positioned anywhere along the length of the gun except where obstructed by the support structure. The entire railgun assembly was situated within the vacuum chamber. 
Figure 2.5 Schematic Diagram of Railgun Assembly
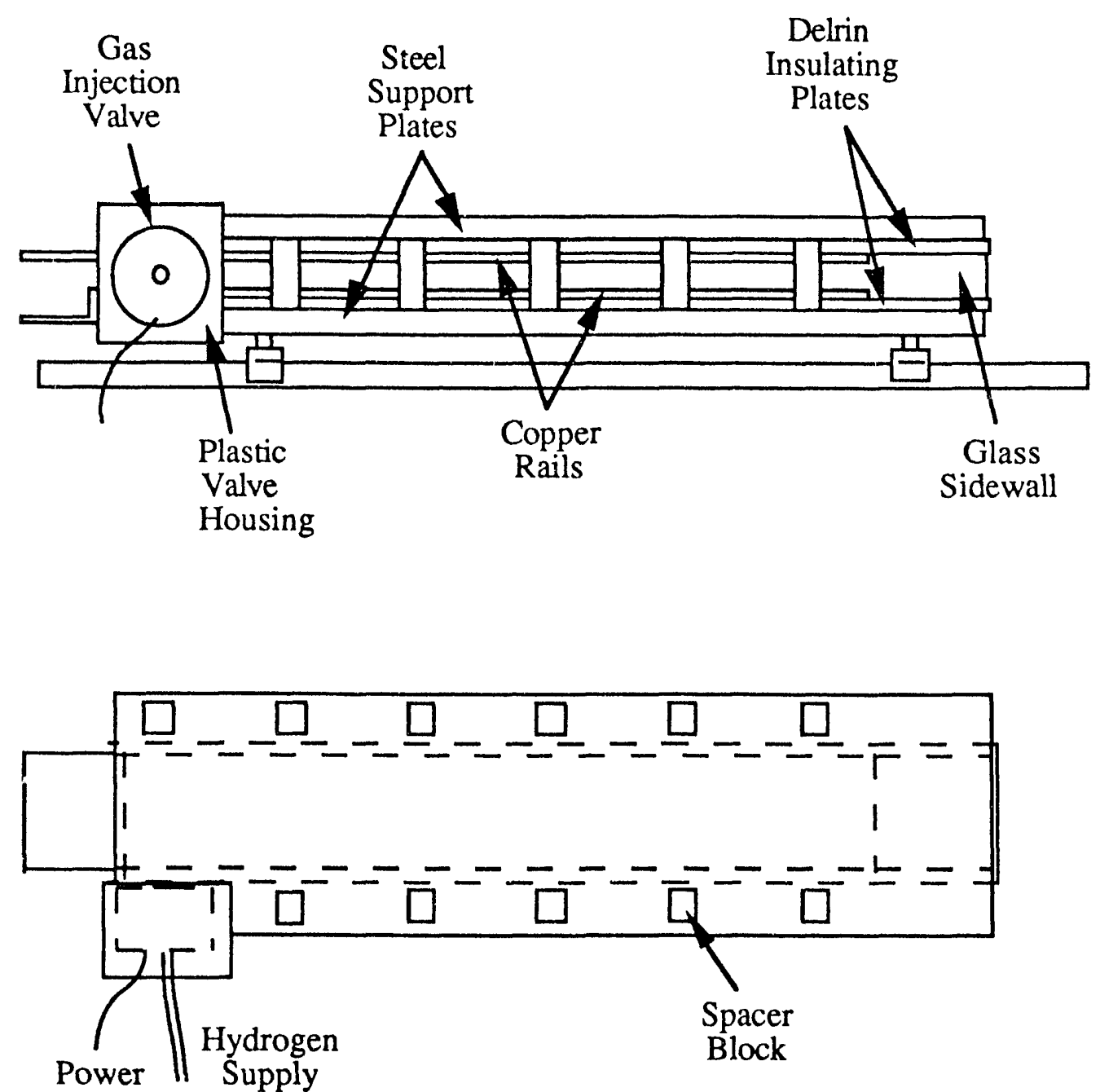

A coaxial cable and high-voltage vacuum feedthrough system was used to connect the railgun to a $510 \mu \mathrm{F}$ capacitor bank. See Fig. 2.6 for a schematic diagram of the feedthrough. This feedthrough was also designed by B. Harden. The cable was constructed by first wrapping an electrically insulated, $25.4 \mathrm{~mm}$ ( 1 inch) diameter, high-voltage power cable with three layers of tinned-copper, 
Figure 2.6 Vacuum Chamber Power Feedthrough

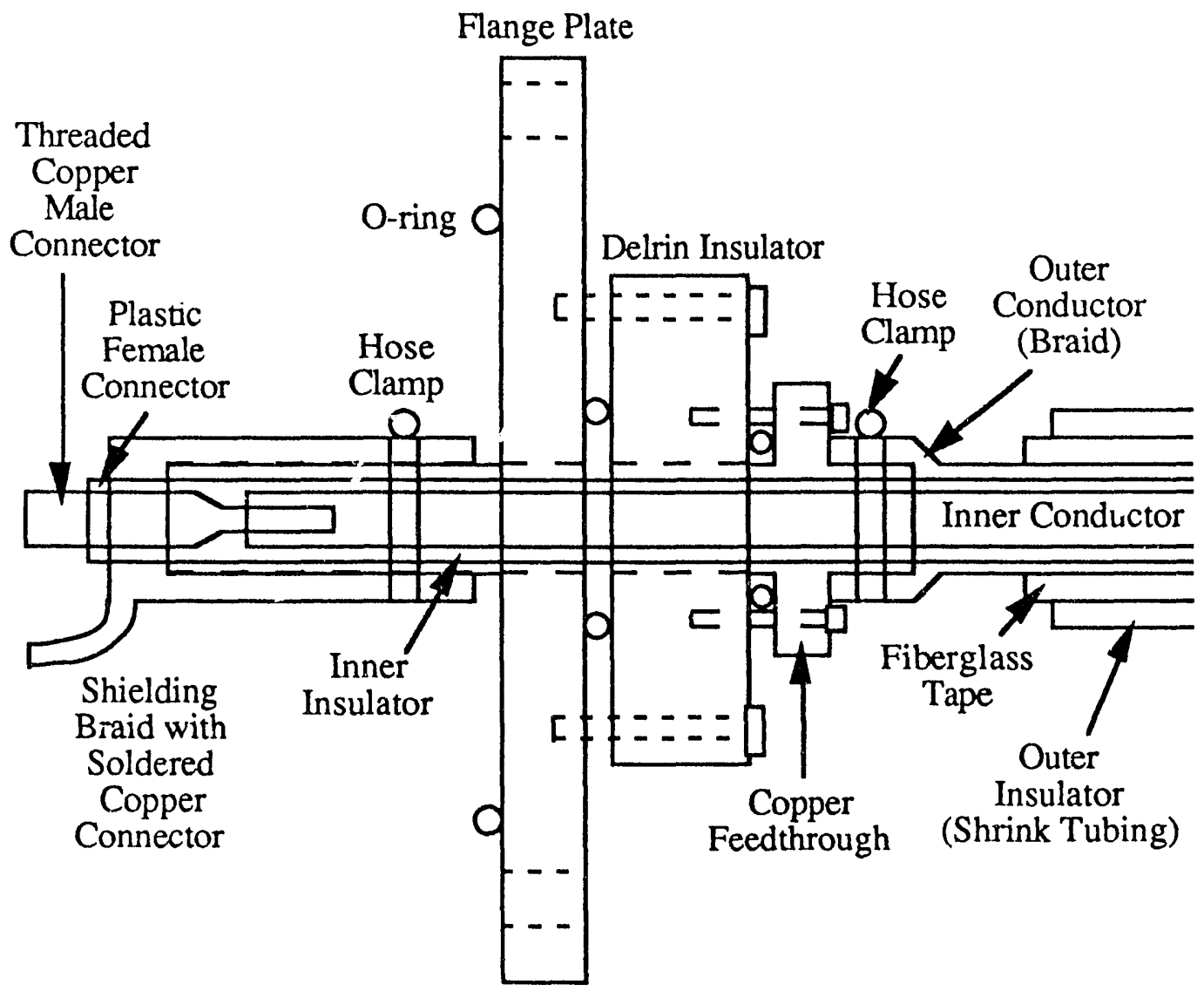

electrical-shielding braid. This braid served as the outer conductor of the cable. The braid was then wrapped with rubber, electrically-insulating, shrink tubing. Finally, the entire assembly was wrapped with high-strength fiberglass tape to constrain it against the repulsive force produced between the inner and outer conductors during the capacitor bank discharge. 
Three different valves were used to inject hydrogen into the railgun. The first valve used was a piezoelectrically-actuated valve. This valve had a variable pulse width (from $150 \mu$ s to continuously open) and was small enough to fit within the limited space available. The advantage it had over other commercially available gas valves was its range of pulse width. It could be operated in several modes including one in which the output signal to the valve mirrored a signal input. This mode of operation allowed the timing and width of the output signal to be supplied by an external source (see Section 2.4). A 10:1, voltage-divided output from the valve driver allowed for verification of the pulse characteristics being sent to the valve. Although this valve possessed many of the features of interest for this experiment it was found through repeated analysis and experimentation to be very susceptible to electromagnetic interference (EMI) and was finally abandoned. Experiments conducted to try to characterized the operation of this valve are described below (see section 2.7).

The results discussed in Chapter 3 were obtained using a solenoid valve constructed by B. Harden. With it we were able to inject about $22 \mu \mathrm{g}$ of hydrogen into the bore of the railgun.

A third valve was used to obtain the results discussed in Chapter 4 . This valve, obtained on loan from Sandia National Laboratories, Albuquerque, New Mexico, was also actuated using a solenoid (see Fig. 2.7 for a schematic diagram of this valve). The volume of the valve plenum $\left(\mathrm{V}=19 \pm 1 \mathrm{~mm}^{3}\right)$ was found by measuring the amount of acetone required to fill it. Using valves 3 and 4 of Fig. 2.4 
Figure 2.7 Schematic Diagram of Solenoid Valve

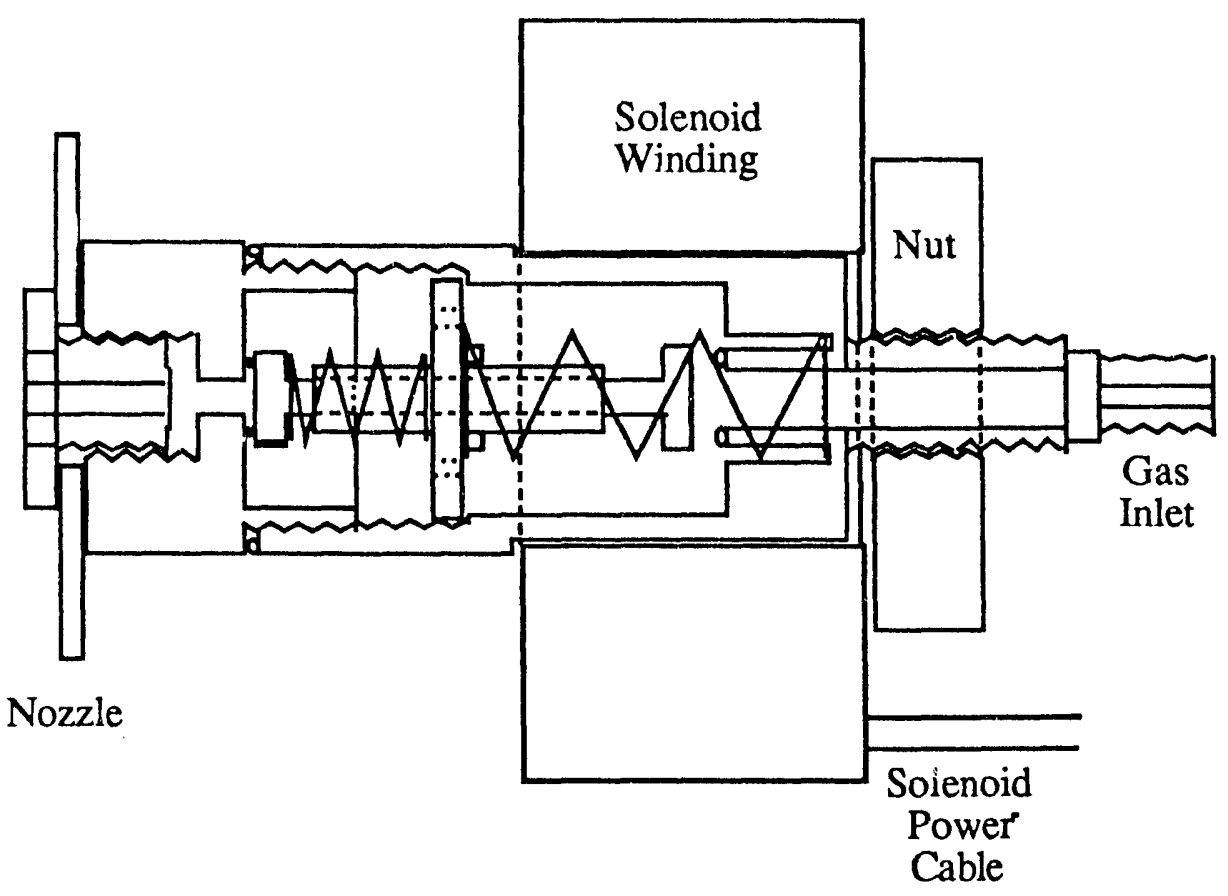

the pressure to the valve could be accurately adjusted, thereby allowing for accurate determination of the injected mass.

\subsection{Power Supply}

Supplying power to the railgun was a complex process. The entire power supply circuit described here was designed and built by J. Allen of The University of Texas at Austin, Center for Electromechanics (CEM). My task consisted only of feeding the power from the capacitor bank to the railgun. 
Power was supplied to the railgun via a $510 \mu \mathrm{F}$ capacitor bank. The capacitor bank was charged using a power supply which had an adjustable output ranging from zero to $22 \mathrm{kV}$. The charging voltage was monitored through a 1000:1 voltage divider. The rate at which the capacitor bank was charged was controlled by adjusting the current output of the power supply. Charging took approximately two minutes.

The capacitor bank was discharged through an ignitron, which is a highvoltage switch. To activate the ignitron a power spike of approximately $200 \mathrm{~V}$ and 4.5 A.was supplied via a firing circuit (see Fig. 2.8) which was powered by a

Figure 2.8 Ignitron Firing Circuit

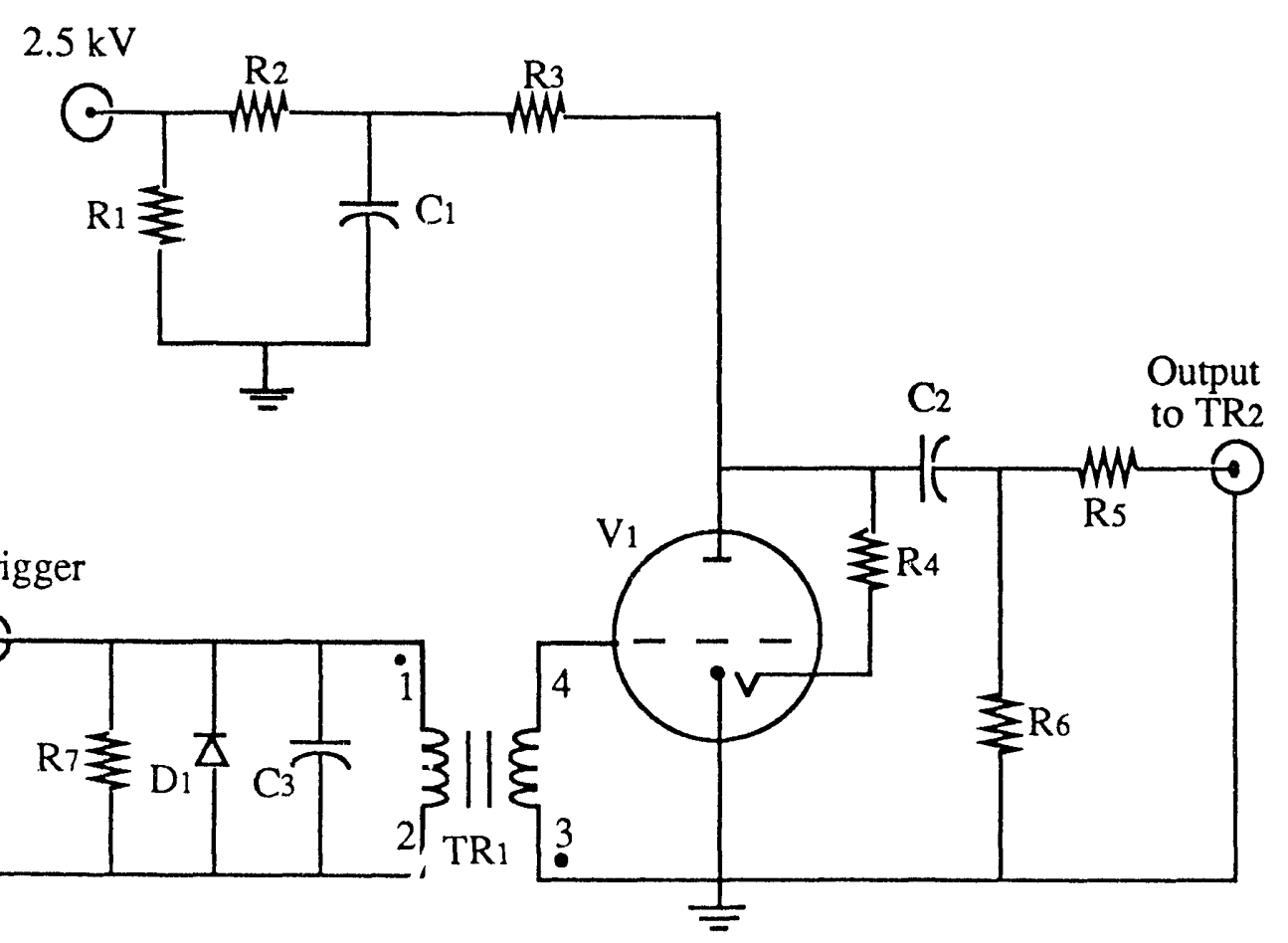


$2500 \mathrm{~V}$ power supply. A different power supply was used to charge the capacitor bank. The power spike supplied by the firing circuit was sent to the ignitor within the ignitron. The ignitor is simply a sharply tipped metallic electrode which is partially submerged in a pool of mercury. When the current flows through the ignitor and into the mercury pool, some of the mercury is ionized, thereby forming a plasma through which current can flow. This plasma formation allows current to flow through the ignitron, discharging the capacitor bank. See Fig. 2.9 for a schematic diagram of the entire capacitor bank system.

\section{Timing Circuit}

Sequencing of the experiments was controlled by a timing circuit designed and constructed by J. Allen of CEM (see Appendix 1 for the circuit diagram). This circuit has seven optically-isolated output channels, two trigger outputs ("Trig" and " $\mathrm{T}_{0}$ ") and five others ("T $\mathrm{T}$ ", " $\mathrm{T}_{\mathrm{B}}$ ", "T $\mathrm{T}_{\mathrm{C}}$, "T $\mathrm{T}_{\mathrm{D}}$, and "TWD") which could be individually delayed from the trigger signal by dialing in the desired time (timing is adjustable in $1 \mu$ s increments from 0 to $9999 \mu \mathrm{s}$ ). The trigger outputs were delayed by three seconds from the pressing of the firing button located on the face of the timing circuit box to allow the experimenter time to stand clear of all electrical equipment before discharge of the capacitor bank. The output voltage of $\mathrm{T}_{\mathrm{A}}$ was designed to drive a separate optical isolation circuit (see Appendix 1 for the circuit diagram), the output of which was in turn used to supply the trigger signal to the ignitron which discharges the capacitor bank. The separate optical isolation circuit insured that no high-voltage spikes could travel back to the timing box and/or data acquisition systems. $T_{B}-T_{W D}$ are TTL level outputs used to sequence other events 
Figure 2.9 Capacitor Bank System

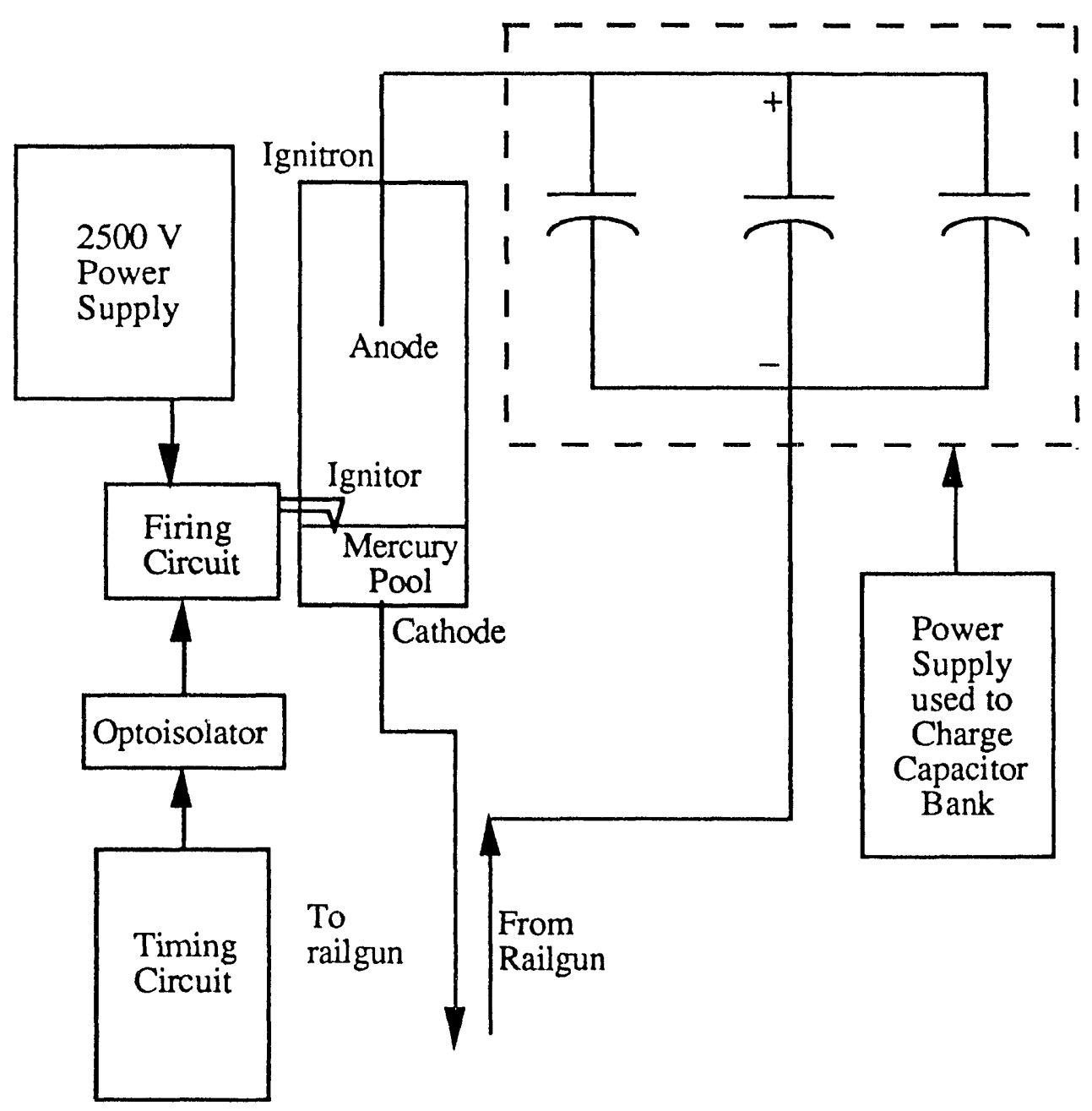

in the experiment, including the triggering and pulse duration of the piezoelectric valve used to inject hydrogen into the railgun and the triggering of the spectrographic equipment and data acquisition system. The outputs from channels $T_{A}-T_{D}$ are step functions, switching from off to on at the delay specified. TWD turns on at the delay specified by $T_{D}$, and turns off at the time specified on its own dial. 


\subsection{Data Acquisition System}

The heart of the data acquisition system was a Compaq $386 / 20$ personal computer. This computer was chosen primarily because of its high speed (important for doing graphics work) and the reputation for quality of its manufacturer. An 80387 coprocessor was also purchased, which greatly increased the speed of the computer. An additional two Mbytes of memory were also installed in the computer to further improve its graphics capability. This computer was used to record and store data collected and digitized on two digital waveform recorders (LeCroy 6810). These waveform recorders had several advantages over other commercially available analog-to-digital converters. Each one could record up to 512 kbytes of data in the following configurations: one channel at up to $5 \mathrm{MHz}$, up to two channels at up to 2 $\mathrm{MHz}$, or up to four channels at up to $1 \mathrm{MHz}$. No other system was found to possess both this amount of memory and configuration flexibility. Also, these recorders allowed for input signals of up to $100 \mathrm{~V}$ in amplitude to be digitized with no external conditioning. This was probably the single greatest asset of the 6810 's, since in an experimental environment such as ours voltage spikes of this size can easily occur. LeCroy "Catalyst" software was used to drive the 6810's and was found to be easy and flexible to use.

Electromagnetic interference (EMI) was found to be a major hindrance in the acquisition of noise-free data from our diagnostic equipment. Therefore, we went to great lengths to eliminate this problem. The entire data-acquisition cabling system was shielded using copper tubing and pipe. Two copper boxes containing banks of male BNC connectors were built to act as junctions between the data acquisition cables from the various diagnostic instruments, and the dedicated, twisted-pair, 
data-transmission cables running to the data acquisition system described above. This arrangement allowed for flexibility in how the data acquisition cabling systems were configured. It was found that grounding the shielding system at a point far away from the capacitor bank allowed for acquisition of the cleanest signals. We reasoned that this phenomenon occurred because ground loops from the capacitor bank to the shielding system diminished in magnitude with distance from the capacitor bank. We also found that twisted-pair cables were much less sensitive to noise than were coaxial cables.

\subsection{Diagnostic Equipment}

\subsubsection{Rogowski coil}

A Rogowski coil is a toroidally-configured coil used to measure the rate of change of the current passing through a conductor looped through its center (see Fig. 2.10). By integrating the signal obtained frorn a Rogowski coil one can calculate the current passing through the conductor. We used a Rogowski coil to measure the current flowing in the inner conductor of the coaxial cable connecting the capacitor bank to the railgun during the capacitor bank discharge. The coil was calibrated by shorting the capacitor bank with a current viewing resistor (CVR), which is a precision shunt. The CVR is used by monitoring the voltage drop across the shunt's known low resistance, allowing accurate calculation of the current present in high current circuits (see Appendix I for specifications of the Rogowski coil and the CVR). 


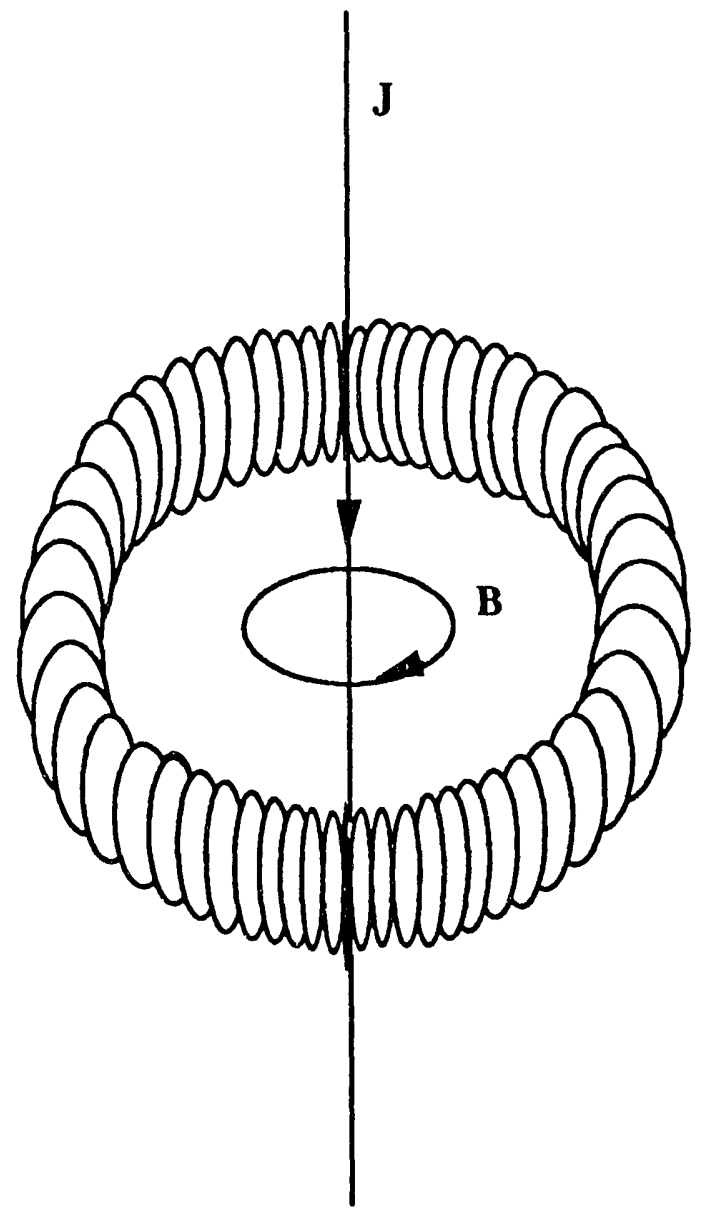

Figure 2.10 Schematic Diagram of Rogowski Coil

\subsubsection{Magnetic Pick-up Coils (ํㅗ-probes)}

The $\stackrel{\circ}{\mathrm{B}}$-probes were constructed using 15 turns of magnet wire wrapped around plastic screws $(6.3 \mathrm{~mm}$ diameter $)$. The axes of the probes were oriented parallel to the direction of motion of the plasma; this orientation measures the plasma- 
armature current passage and ignores the effects of the current in the rails (see Fig. $2.11)^{2.2}$ The $\stackrel{\circ}{\mathrm{B}}$-probes were connected to the data acquisition system via shielded, twisted-pair wire cables through the shielded cabling system described above. We found that terminating the wire cables with $50 \Omega$ resistors reduced the ringing on the probe signals. Also, the cables were set up so that the data acquisition system measured the differential voltage across the probes. This configuration further reduced the EMI on the signals.

Figure 2.11 B-dot Probe Orientation

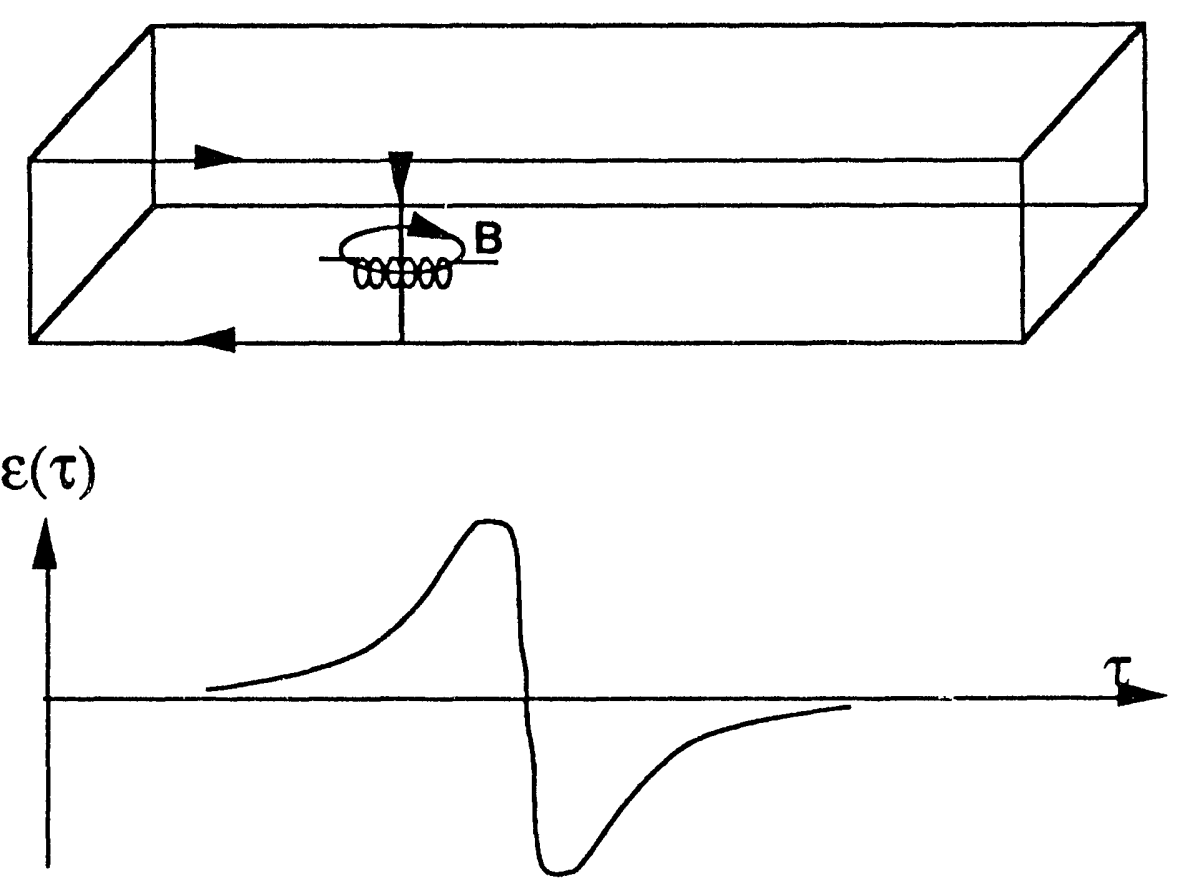




\subsubsection{Phototransistors}

The light from the plasma was coupled to phototransistors located outside the vacuum chamber via $1 \mathrm{~mm}$ core diameter optical fibers. The fibers were oriented tolook through the glass side-walls of the railgun perpendicular to the direction of motion of the plasma. The phototransistors and the associated circuitry (see Fig. 2.12 for the circuit diagram) were located in a copper enclosure situated on the base plate of the vacuum chamber support. Four optical fibers could be individually positioned along the length of the railgun, with the output of each going to a separate phototransistor. The phototransistor circuit transduced and amplified the light output to a voltage output, which was then recorded on the Lecroy data acquisition system The phototransistor circuit allowed the output voltage of each phototransistor to be individually adjusted, so that for a given input light signal all four phototransistors gave equal outputs.

The $\stackrel{\circ}{\mathrm{B}}$-probes and the optical fibers coupled to the phototransistors were mounted on posts attached to optical rails which run along either side of the railgun. The probes and fibers could be positioned anywhere along the axis of the railgun, except where obstructed by the railgun support structure. For ease of positioning, two aluminum probe holders were constructed. These holders had $25.4 \mathrm{~mm}$ ( 1 inch) square cross sections and were 1 meter (40 inches) in length. Holes into which the probes could be inserted were machined on $76.2 \mathrm{~mm}$ ( 3 inch) centers. This arrangement allowed for accurate positioning of the probes in that the position of only one point had to be measured and adjusted for each set of probes 
Figure 2.12: Phototransistor Circuit

Individual Photransistor Circuit
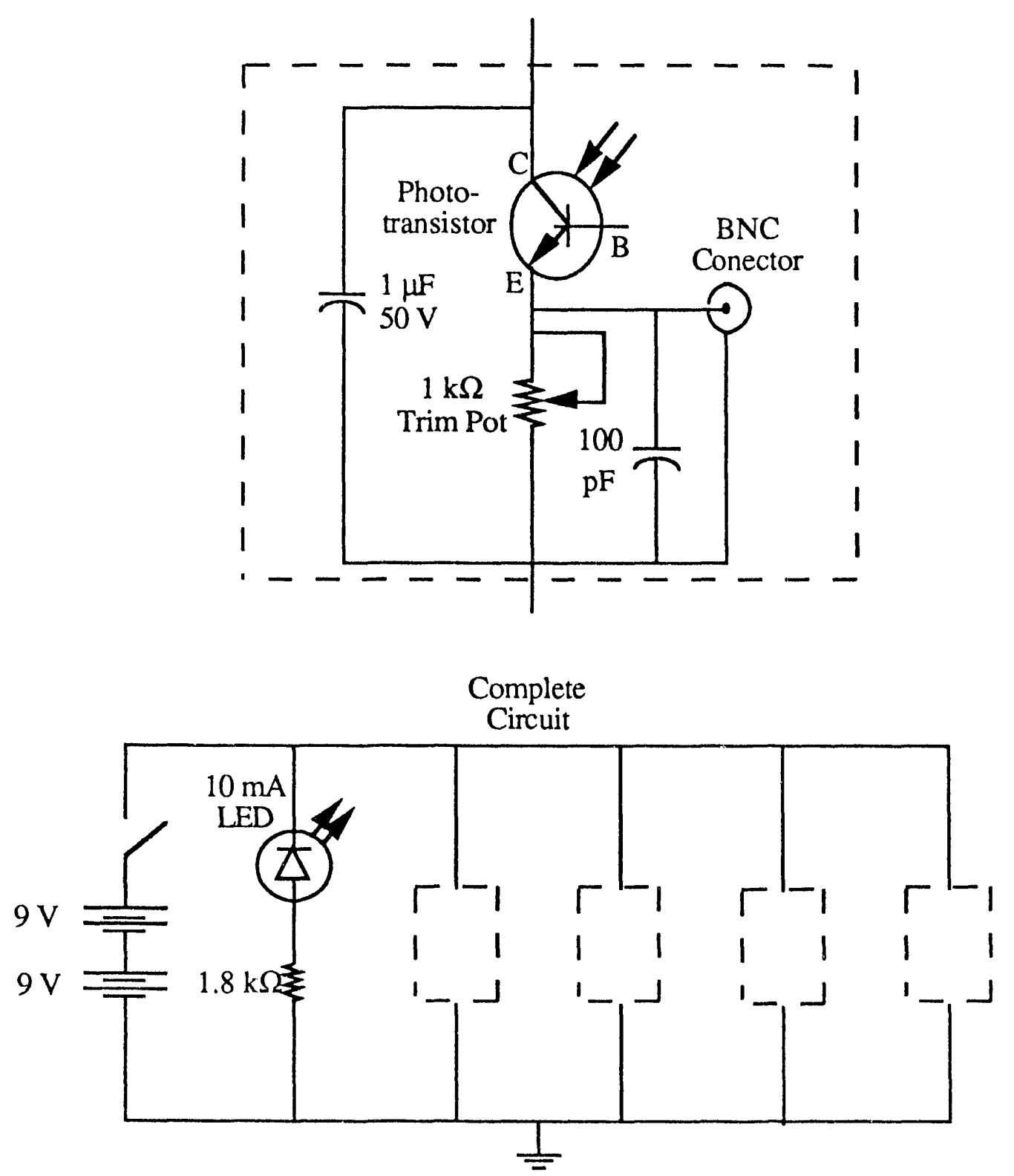
rather than positioning each probe individually. A minor drawback to this scheme was the reduction in flexibility in probe positioning.

Both $\stackrel{\circ}{\mathrm{B}}$-probes and phototransistors were to be used to detect the time at which the plasma armatures passed fixed points along the railgun bore. In order to use these two very different diagnostic tools to make the same measurement it was felt necessary to determine the correspondence between their outputs. To do this, pairs of $\stackrel{\circ}{\mathrm{B}}$-probes and phototransistors were positioned across from each other at several locations along the sides of the railgun. It was found that the zero crossing of the $\stackrel{\circ}{\mathrm{B}}$-probe output always occurred within $1 \mu$ s of the peak of the phototransistor output. This is the limit of precision in time measurements of the data acquisition system (1MHz/channel digitizing rate). Because of this correspondence the peaks of the phototransistor outputs and the crossover points of the $\stackrel{\circ}{\mathrm{B}}$-probe outputs were used to fix the plasma front arrival time for plasma velocity measurements.

\subsubsection{Photodiode array, spectral data acquisition system}

All spectral data were collected using an optical fiber (numerical aperture $(\mathrm{NA})=0.5)$ mounted in the same way as those coupled to the phototransistors. The output of this fiber was coupled to the input of a $0.22 \mathrm{~m}$ spectrometer $(\mathrm{NA}=0.125)$ via a pair of achromats. The spectrometer was equipped with an adjustable entrance slit and either a 300 groove/mm or a 1200 groove/mm grating. For the experiments described in Chapters 3 and 4 a 512 element, intensified, gatable, linear, photodiode array detector (PDA) was positioned at the output slit of the spectrometer for data acquisition (see Ref. 2.3 for complete specifications of this system). The output of the PDA was collected using a Tracor Northern TN-6500 optical multichannel 
spectrum analyzer (see Ref. 2.3 for complete specifications of this system). Data was acquired by setting the scan time for the PDA to 0.5 seconds at the trigger of the experiment. However, data was only acquired when the accompanying pulser sent a signal to the intensifier. This process was controlled by sending a signal from the timing circuit described above to a waveform generator, which in turn sent a signal to the pulser. The waveform generator allowed us to adjust the width of the pulse sent to the pulser.

For the experiments described in Chapter 5 an intensified, gatable, two dimensional CCD camera was used (see Chapter 5 for the details of this system).

Alignment of the spectral acquisition systems was accomplished using a Helium-Neon laser. The laser output was focussed on the fiber optic cable used for spectral acquisition inside the vacuum chamber. The fiber output was focussed on the inlet slit of the spectrometer via a pair of achromatic lenses. These lenses were used to reduce chromatic aberrations in the image on the entrance slit.

\subsection{Hydrogen pressure experiment}

It was decided early in the experiment that knowledge of the hydrogen pressure distribution within the railgun prior to its being fired was of interest. Marshall. 1.6 conducted an experiment in which he determined the pressure distribution of deuterium, with time, along the length of a $9.2 \mathrm{~cm}$ diameter tube. For a valve plenum pressure of about one atmosphere he found that the gas propagated in vacuum at a velocity close to its speed of sound. He concluded that the gas propagation speed was limited by the molecules being re-thermalized and randomized

in direction by collision with the walls of the tube. Burkhardt and Loveberg 2.4 made 
similar measurements, also using deuterium at about one atmosphere back pressure. By measuring the valve plenum volume and pressure they were able to normalize the ion gauge pulse shapes and obtain an absolute density distribution of the gas at the time of firing their railgun.

Because the piezoelectric valve described in Section 2.2 allowed back pressures up to ten atmospheres, much higher than the pressures used in the above experiments, it was decided that a similar measurement of the hydrogen distribution in our railgun would be of interest. The primary goal of this experiment was the determination of the period of time available betwee the opening of the valve and hydrogen reaching the muzzle of the railgun. This parameter was necessary to time the discharge of the capacitor banks correctly.

A full-size mock-up of the railgun was designed and built in which to conduct this experiment, and an ionization gauge with a $3 \mu$ s response time was purchased to measure the hydrogen propagation (see Fig. 2.13 for a schematic diagram of this setup). The piezoelectric valve was installed at the closed end of the mock-up. At ten, equally spaced positions along the length of the mock-up, holes were cut where the ion gauge could be inserted. The unused holes were filled with plugs machined to match the inside walls of the railgun.

Results from this experiment showed that about one millisecond elapsed between valve triggering and hydrogen reaching the end of the railgun. Quantitative pressure distribution results were not obtained due to fluctuations in the output of the ion gauge, even on successive valve firings under constant conditions. It was never 
determined whether the non-repeatability resulted from jitter in the valve response or in response of the ion gauge.

This apparatus was also used to Gutermine the elapsed time between the trigger of the Slapshot driver and arrival of the hydrogen at the position nearest the breech of the railgun. This information was of interest in the determination of when to initiate discharge of the railgun. This time difference was found to be about 3.5 ms. 


\section{CHAPTER 3}

\section{RESULTS AND DISCUSSION OF EXPERIMENT 1}

\subsection{Introduction}

The set of experiments described here was conducted primarily for the purpose of exercising all of the components in this rather complex experiment as a coherent group. Each of these components had been extensively tested prior to this set of experiments and it was here that all of the pieces were integrated. Measurements of the plasma armature velocity, temperature, electron density, and composition were conducted. The latter three of these parameters were measured at several points within the plasma armatures to give profiles of their values. These profile measurements were obtained using short $(2 \mu \mathrm{s})$ snapshots of the spectra from the plasma armature taken with the photodiode array. The velocity was characterized using the $\stackrel{\circ}{\mathrm{B}}$-probes and phototransistors described in Chapter 2.

\subsection{Experimental Setup}

The railgun described in Chapter 2 was assembled and placed inside the vacuum chamber. The valve used to inject the hydrogen into the breech of the railgun for this set of experiments was the solenoid valve built by B. Harden. The $\stackrel{\circ}{\mathrm{B} \text {-probes }}$ and the optical fibers used to transmit light to the phototransistors were positioned as shown in Fig. 3.1. One fiber was placed downstream of the muzzle so that the plasma velocity could be determine at this 
Figure 3.1 B-dot probe and optical fiber positions.

Distances s'lown are in meters

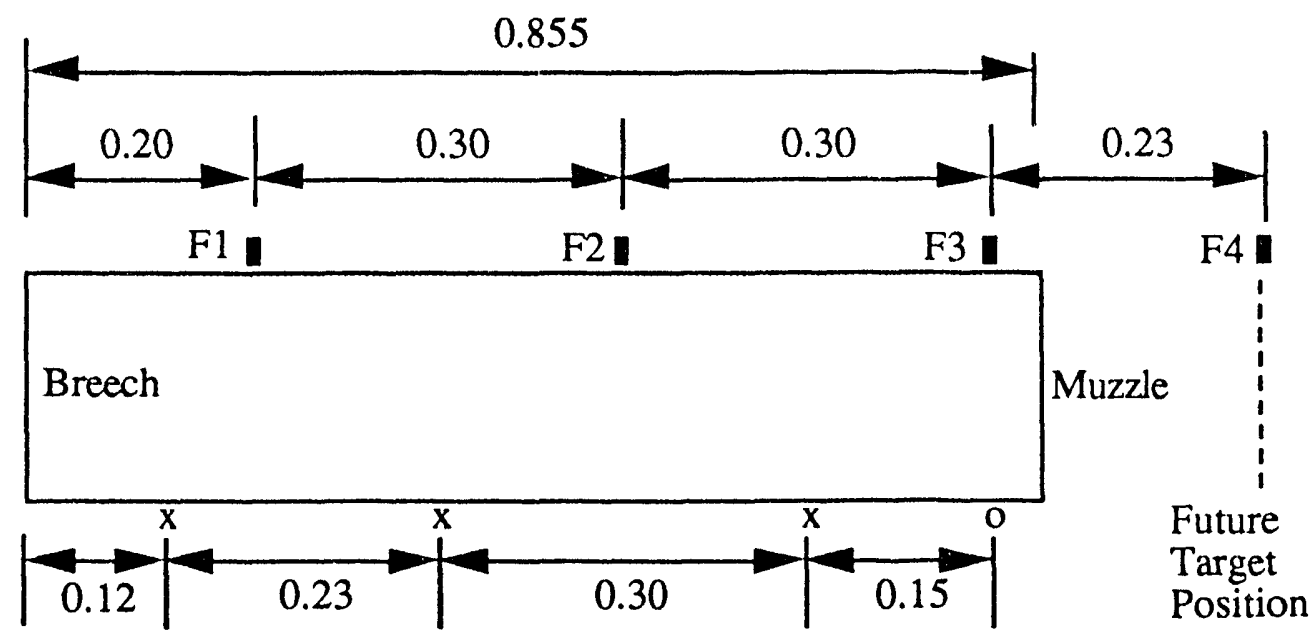

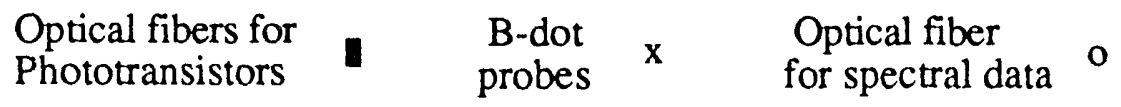

location. The outputs from the $\stackrel{\circ}{\mathrm{B}}$-probes and phototransistors were coupled to the LeCroy data acquisition system. In addition to the three $\stackrel{\circ}{\mathrm{B}}$-probes and four phototransistors, the pulse being sent to the intensifier of the photodiode array was also recorded. Because eight separate signals were being recorded, the maximum digitizing rate available was $1 \mathrm{MHz}$.

Spectral data were collected using an optical fiber mounted as described above and located near the muzzle opposite F3 (see Fig. 3.1). This was done so that the spectral measurements could be correlated with the plasma emission at this axial 
location. For this set of experiments the fiber input axis was positioned midway between the rails. The output of this fiber was coupled to the $0.22 \mathrm{~m}$ spectrometer.

\subsection{Experimental Sequence}

After the railgun was assembled and all of the diagnostic probes attached, it was placed inside the vacuum chamber, which was then pumped down to about $1 \mu$ Torr. The gas injection valve was then pressurized with hydrogen to 760 Torr. The gas valve driver and the capacitor bank and its associated firing circuit were then charged. For this set of experiments the capacitor bank was charged to $3 \mathrm{kV}$ because at higher voltages much larger restrike armatures (see description below) were found to occur. At this point the railgun was ready to be fired. Figure 3.2 shows how all of the components described in Chapter 2 were interconnected for this experiment.

Figure 3.3 shows the timing sequence of the experiment. The letters denote the output channels of the timing circuit described in Chapter 2. First, a pulse was sent to the gas valve driver $\left(T_{D}\right)$. Between this trigger and actuation of the valve there was a delay of about $4.95 \mathrm{~ms}$ so at this time triggers were sent to the ignitron firing circuit $\left(T_{A}\right)$, the data acquisition system $\left(T_{B}\right)$, and the waveform generator $\left(T_{C}\right)$. The delay shown as SG represents the delay between discharge of the capacitor bank and acquisition of the gated spectra obtained using the photodiode array. This delay was set using the waveform generator. Both the timing and the width of this pulse could be adjusted. The width was set at $2 \mu$ s for this set of experiments. The delay was adjusted to obtain spectra from several points in the plasma armatures. 
Figure 3.2 Experimental Apparatus

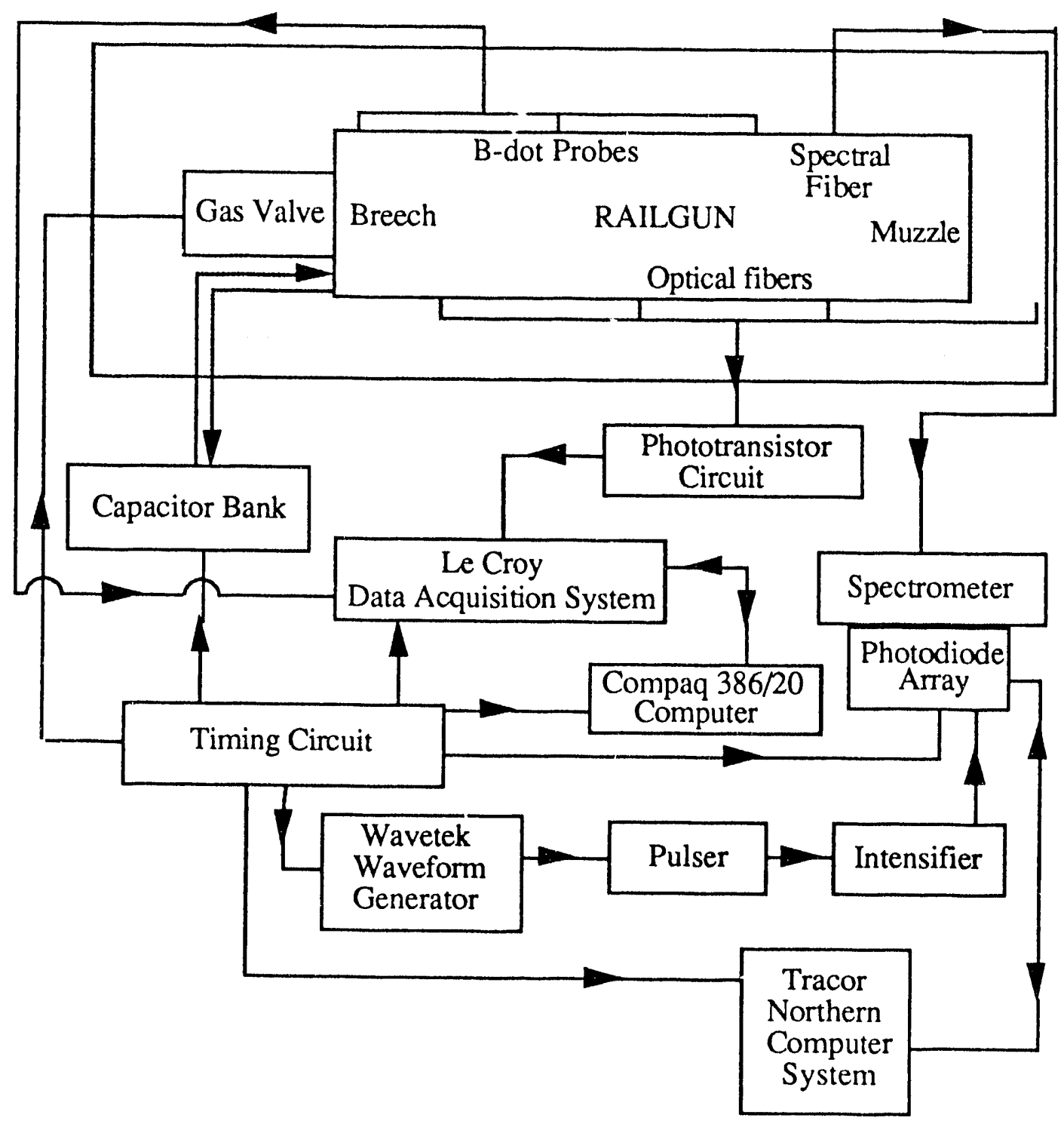


Figure 3.3 Experimental Timing Sequence

TD

Pulse to gas valve

$\mathrm{TA}_{\mathrm{A}}$

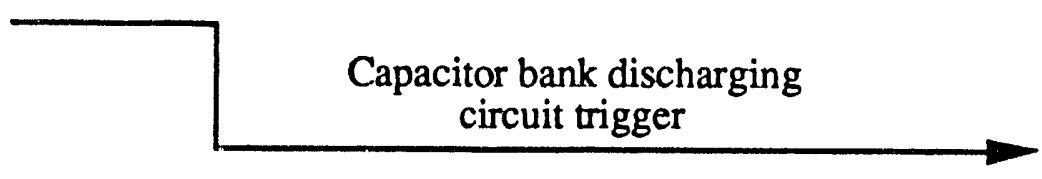

TB

Data acquisition system trigger

Tc

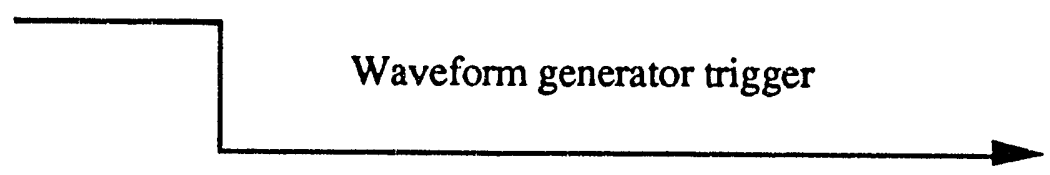

SG

Photodiode array intensifier pulse

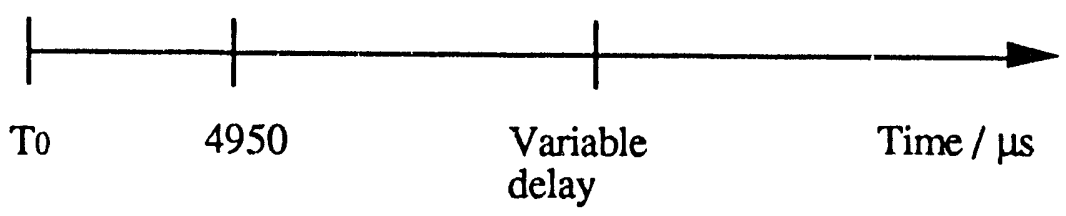




\subsection{B -Probe and Phototransistor Measurements}

Two distinct plasma armatures were detected with the phototransistors. Figure 3.4 shows a sample of the output from the phototransistor connected to the output of the fiber at F3, $5.5 \mathrm{~cm}$ upstream of the muzzle. Light from the first plasma armature was detected by all four phototransistors $45 \mu \mathrm{s}$ after the capacitor bank started discharging. This indicated that the plasma filled the entire railgun and extended beyond the muzzle at this time. Every phototransistor showed a sharp rise initially, corresponding to the arrival of the plasma front at the corresponding fiber location. There was a clear progression in rise times indicating the motion of the plasma down the gun. The trailing edge of the armature could also be seen to

Figure 3.4 Output of phototransistor F3 of Fig. 3.1 relative to time of arrival of primary plasma armature front.

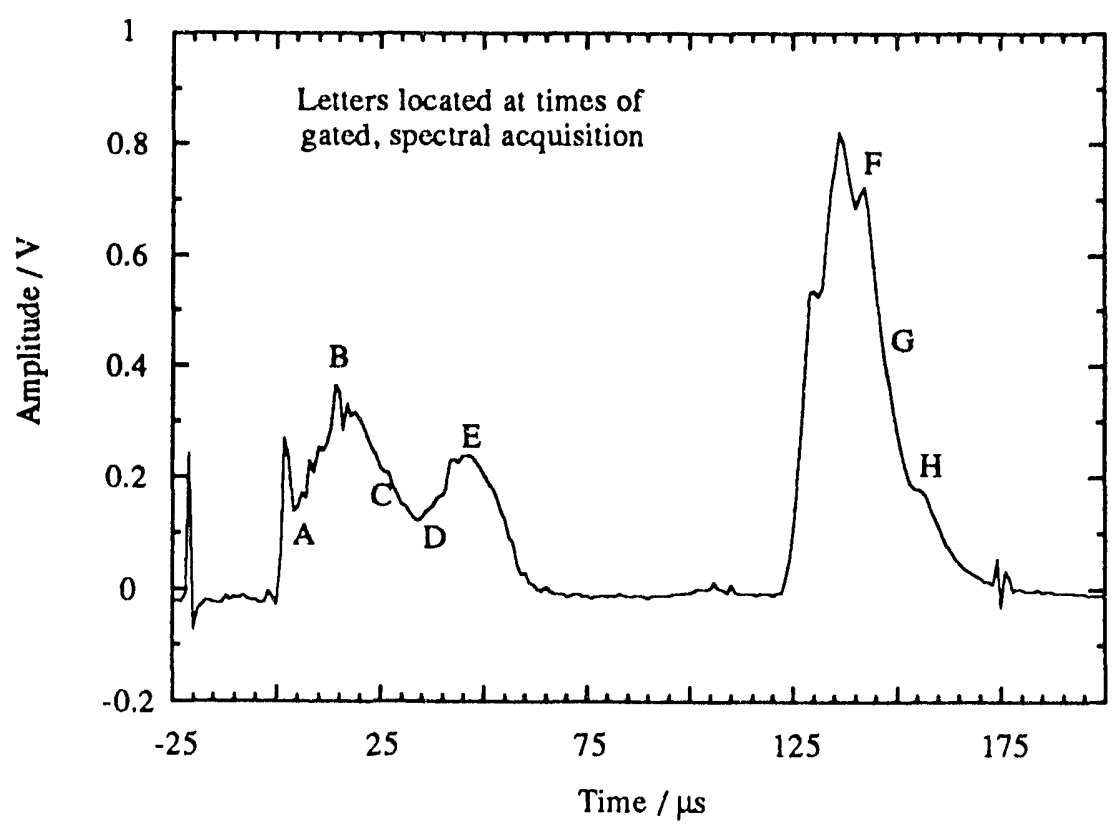


progress down the barrel, although much less distinctly than the leading edge. By comparing the outputs of phototransistors coupled to fibers positioned near the breech and the muzzle it was determined that the restrike armature began to form immediately after the first armature left the railgun. The restrike armature did not appear to travel beyond the muzzle and was not detected by the phototransistor connected to the fiber stationed $18 \mathrm{~cm}$ beyond the muzzle.

Figure 3.5 shows a sample of the output obtained from the $\stackrel{\circ}{\mathrm{B}}$-probe located $20.5 \mathrm{~cm}$ upstream of the railgun muzzle. The integrated output of the Rogowski coil is also shown. Again, it can be seen that distinct primary and restrike

Figure 3.5 Output of B-dot probe positioned $20.5 \mathrm{~cm}$ upstream of railgun muzzle and integrated Rogowski coil output (railgun current) vs time.

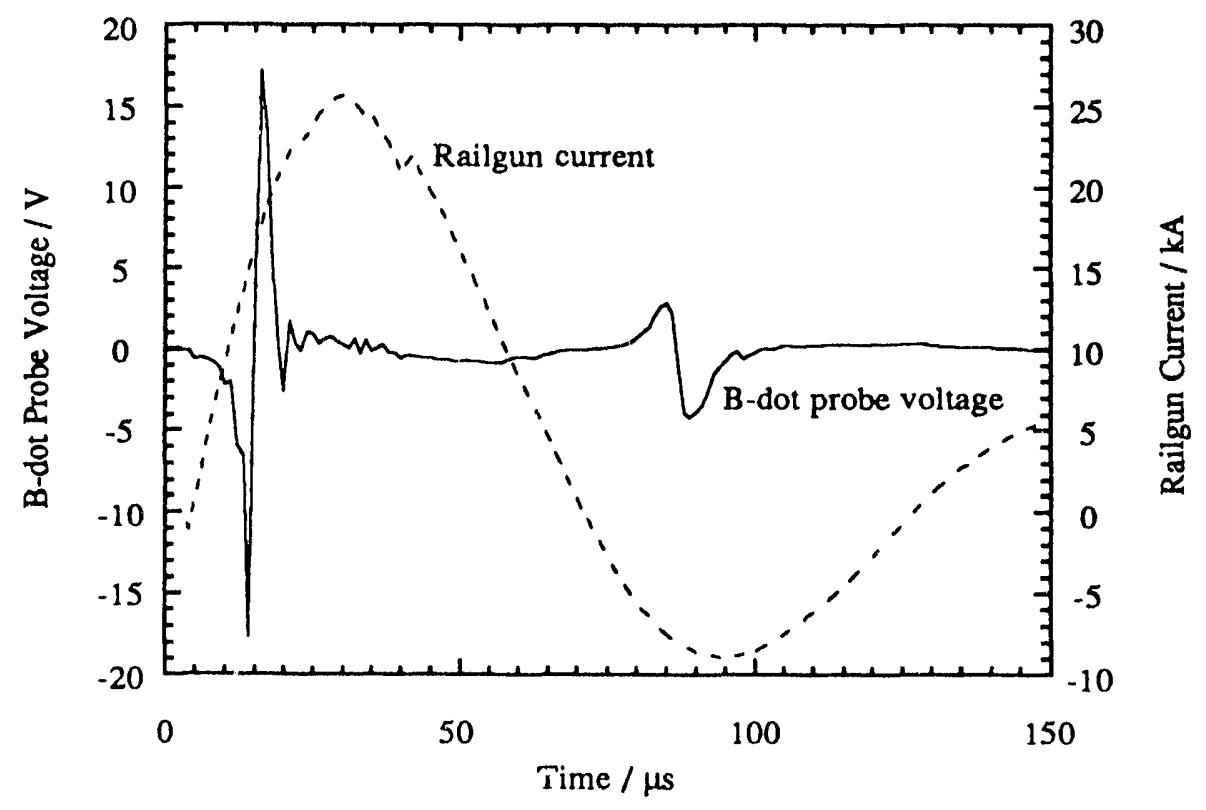


armatures pass the $\stackrel{\circ}{\mathrm{B}}$-probe. The $\stackrel{\circ}{\mathrm{B}}$-probe output of the second armature is inverted with respect to the first. This behavior can be explained by noting that the Rogowski coil output showed that the railgun current had reversed polarity between arrival of the two armatures. Figures 3.6 and 3.7 show the times at which the first and second armatures respectively, arrive at succeeding positions down the railgun. The data from the first phototransistor were omitted for the first armature because the signal-tonoise ratio was so small that the arrival time could not be determined accurately. As mentioned previously, the second armature was not observed $18 \mathrm{~cm}$ beyond the muzzle.

Figure 3.6 Time of arrival of primary plasma armature front vs distance from railgun breech.

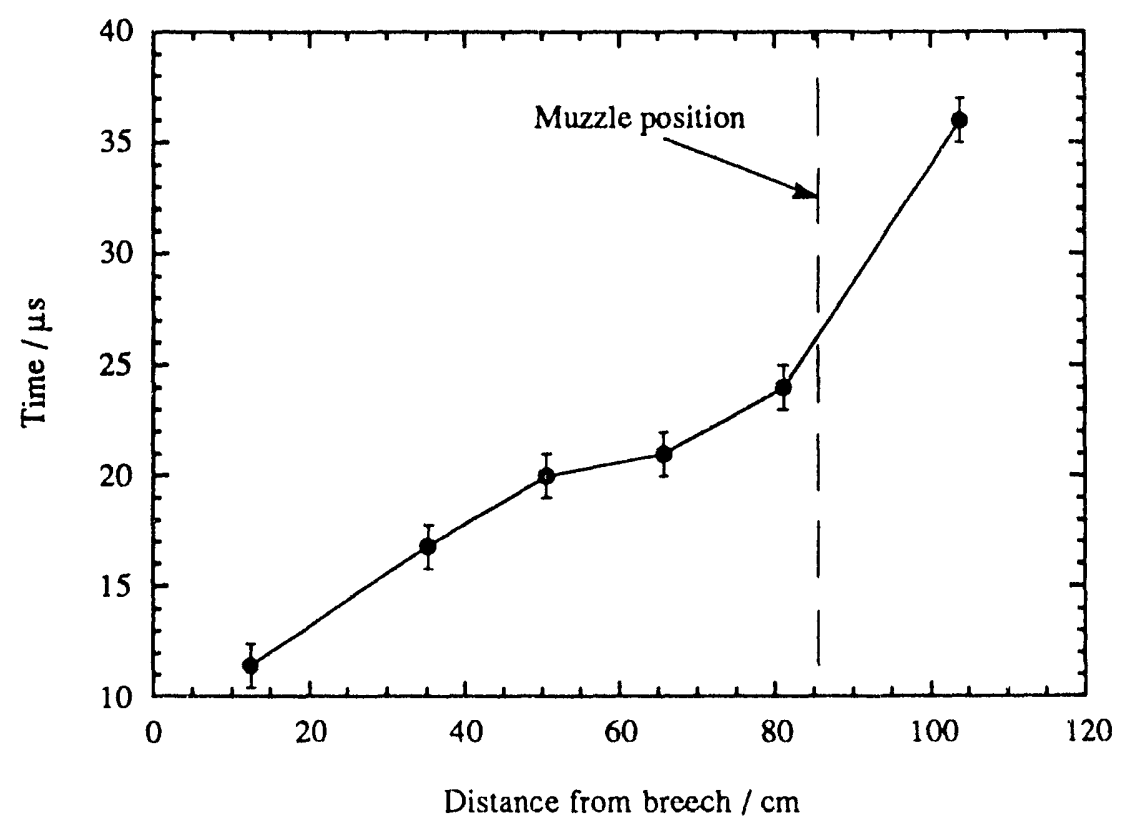


Figure 3.7 Time of arrival of restrike plasma armature vs distance from breech.

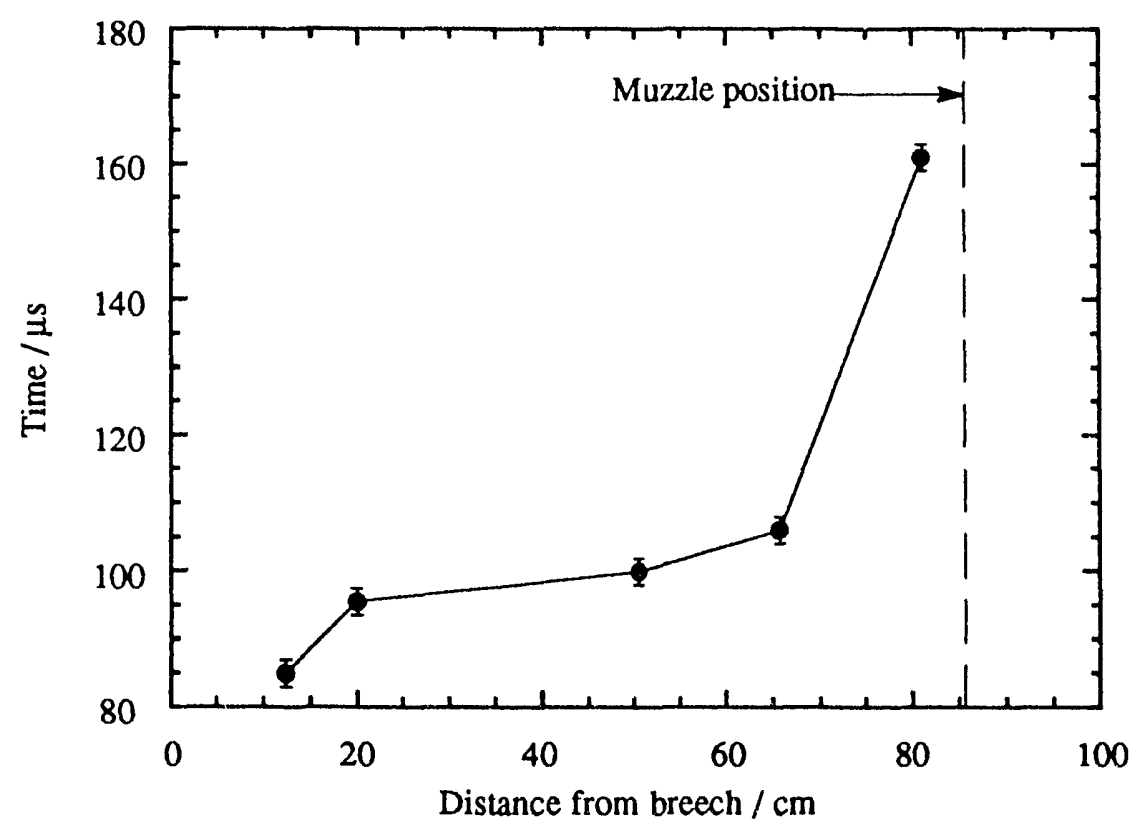

The average velocity between the probes was calculated from these data (see Fig. 3.8). It can be seen from Fig. 3.8 that the first armature attained a maximum front velocity of about $150 \mathrm{~km} / \mathrm{s}$ at a distance of $0.6 \mathrm{~m}$ from the breech, but slowed down to less than $20 \mathrm{~km} / \mathrm{s}$ by the time it traveled $0.2 \mathrm{~m}$ beyond the muzzle. The second armature accelerated to a maximum velocity of $35 \mathrm{~km} / \mathrm{s}$ at a distance of $0.35 \mathrm{~m}$ from the breech and then decelerated as it approached the muzzle (see Fig. 3.8). It is interesting to note that the velocity of each plasma armature reached a maximum, and then decreased with distance from the breech. The error in these velocity measurements was estimated to be $\pm 15 \%$. 
Figure 3.8 Average velocities of the primary and restrike armatures vs position.

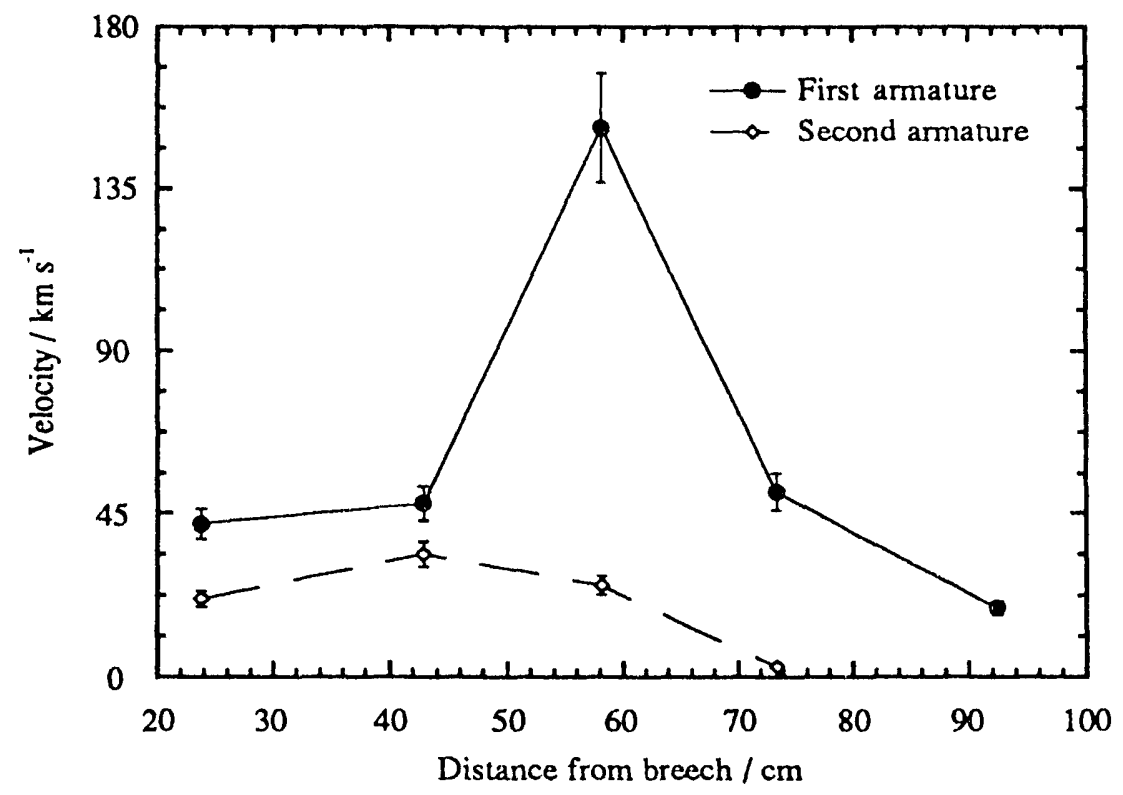

\subsection{Composition of Plasma Armatures}

NBS tables ${ }^{3.1}$ were used for spectral identification and lines of $\mathrm{H}, \mathrm{O}^{+}, \mathrm{N}^{+}$, and $\mathrm{Si}^{2+}$ were identified in the plasma. To identify a species, the strongest line in the possible multiplet of the species was looked for and, in case it was absent, the rest of the transitions in that multiplet were ruled out.

The letters above the curve in Figure 3.4 show the times at which gated spectra were acquired, and Fig. 3.9 shows the spectrum acquired from the center of the first maximum in emission from the first plasma armature (point B on Fig. 3.4). From analysis of the spectrum it was determined that this emission comes from $\mathrm{Si}^{2+}$, 
Figure 3.9 Spectrum acquired at time B of Fig. 3.4.

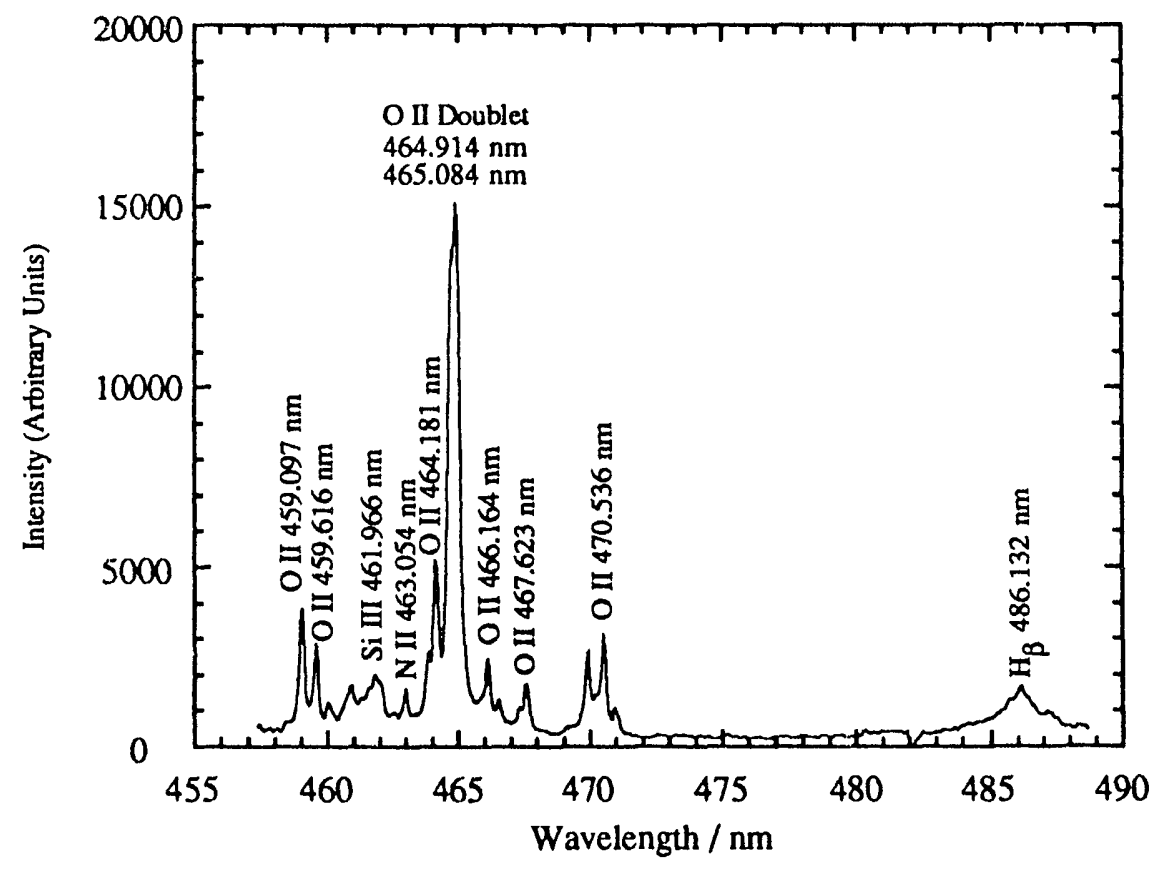

$\mathrm{O}^{+}, \mathrm{N}^{+}$, and neutral $\mathrm{H}$ atoms. Neutral $\mathrm{Cu}$ is probably also present in small amounts as a few weak $\mathrm{Cu}$ I transitions were observed. The second emission hump from the first armature is almost entirely $\mathrm{H} \mathrm{I}$ emission suggesting that this section of the plasma consists mainly of neutral hydrogen and $\mathrm{H}^{+}$ions (which do not emit). It is evident from Fig. 3.4 that the restrike armature is more sharply defined than the first armature. Only $\mathrm{H}$ I transitions and weak $\mathrm{Cu}$ I transitions were observed in the spectra obtained from the restrike armature. 
3.6 Optical thickness of the plasma source

By using $2 \mu$ s intensifier gate widths, the photodiode array was set to analyze light from $0.1 \mathrm{~m}$ long slices of the plasma (based on the average plasma front velocity at the point of spectral data collection). These slices are $25 \mathrm{~mm}$ in height and $75 \mathrm{~mm}$ in depth, based on the cross-sectional area of the railgun.

To estimate the optical thickness of the plasma source, a comparison was made of the measured and expected line strength ratios of several transitions from the $\mathrm{O}^{+}$multiplet $\left[2 \mathrm{p}^{2} 3 \mathrm{~s}^{4} \mathrm{P}-2 \mathrm{p}^{2}\left({ }^{3} \mathrm{P}\right) 3 \mathrm{p}{ }^{4} \mathrm{D}^{0}\right]$. The NBS tables ${ }^{3.1}$ list the line strengths within this multiplet, which follow the intermediate coupling scheme. They have been estimated by using the experimental data of Mastrup and Wiese 3.2 and theoretical calculations of Garstang. ${ }^{3.3}$ If a plasma source is optically thin the measured line strength ratios should have the same magnitude and follow the same trends as the NBS values. If the plasma is optically thick, the ratio of the weaker transitions to stronger transitions in a given multiplet will be larger than those listed in the NBS tables. (The absorption coefficient for a given transition is directly proportional to its transition probability. ${ }^{3.4}$ Since the stronger lines have higher transition probabilities they will be absorbed more than the weaker ones.)

In an optically thin plasma the ratio of the emissivities (intensities) of two transitions ( 1 and 2 ) from the same multiplet is given by 3.5

$$
\left(S_{1} / S_{2}\right)=\left(s_{1} / s_{2}\right)\left(\lambda_{1} / \lambda_{2}\right)^{4} \exp \left(-\left(E_{1_{1}}-E_{l_{2}}\right) / k T\right) \cong\left(s_{1} / s_{2}\right)
$$

Here $S$ is the measured intensity of the transition, $s$ is the strength of the transition, $\lambda$ is the wavelength, $E_{l}$ is the energy of the upper level of the transition, $k$ is 
Boltzmann's constant, and $T$ is the plasma temperature. Table 3.1 shows the comparison of the theoretical and experimental line strength ratios of transitions of $\mathrm{O}^{+}$ (multiplet of type 3s-3p) at time B of Fig. 3.4.

Table 3.1 Experimental and theoretical line strength ratios of $\mathrm{O}^{+}$transitions

\begin{tabular}{ccc}
\hline $\begin{array}{c}\text { Wavelength } \\
(\lambda / \mathrm{nm})\end{array}$ & \multicolumn{2}{c}{$\begin{array}{c}\text { Line Strength Ratios } \\
\left(\mathrm{S} / \mathrm{S}_{1}\right)\end{array}$} \\
\cline { 2 - 3 } & $\begin{array}{c}\text { Expected } \\
( \pm 20 \%)\end{array}$ & Measured \\
464.181 & 1 & 1 \\
466.164 & 0.45 & 0.45 \\
467.623 & 0.33 & 0.26
\end{tabular}

Because the measured ratios were not anomalously high it was inferred that the plasma was optically thin at this position within the armature. This conclusion can be extended to other transitions of $\mathrm{O}^{+}$as these transitions are of the same type, i.e., nonresonant transitions among the highly excited states. These transitions were used in the calculation of temperatures via Boltzmann plots (see section 3.8). Similar results were obtained at positions $\mathrm{A}$ and $\mathrm{C}$ in the armature. 


\subsection{Electron-density measurements}

The electron density of a hydrogen plasma can be estimated by measuring the full width at half maximum (FWHM) of the $\mathrm{H} \beta(486.133 \mathrm{~nm})$ transition. ${ }^{3.3}$ However, this measurement uses only two data points, and hence it is more sensitive to error introduced by noise and interference from overlapping lines of other species. It also not account for instrumental broadening, which was found to be significant in this experiment. Therefore it was decided to compare the entire $\mathrm{H}_{\beta}$ profiles with those predicted by the theory of Vidal et al. 3.6

The theoretical $\mathrm{H}_{\beta}$ profiles calculated by Vidal et al. account for Starkbroadening and Doppler broadening only. However, the instrumental broadening of our spectrographic system was measured and its FWHM was found to be about $4 \AA$. To account for this added broadening the theoretical profiles were convolved with the instrumental profile before comparing them with our experimentally measured profiles. Please see Appendix II for a detailed discussion of the procedure used to obtain an accurate comparison between the experimentally-measured and theoretical $\mathrm{H}_{\beta}$ profiles. Although the profiles used for comparison with the railgun data are actually convolutions of the theoretical profiles and the measured instrumental broadening they will be called the "theoretical" profile for convenience.

The experimental $\mathrm{H}_{\beta}$ profiles obtained at times $\mathrm{A}, \mathrm{B}$, and $\mathrm{C}$ were of relatively low amplitude compared with the other lines present in the spectra. The gain of the intensifier on the photodiode array (PDA) was adjusted so that these other lines approached the saturation point of the PDA. Figure 3.9 is the spectrum obtained in this way at time B. Once the gain was adjusted to give the maximum spectral intensity 
output short of saturation it was fixed. All of the spectra were obtained with this same gain setting. At times A, B, and C there were strong interference lines overlapping the $\mathrm{H}_{\beta}$ line. Figure 3.10, which is the spectrum acquired at time $\mathrm{F}$, illustrates this point (compare this figure with Fig. 3.9). Because of these overlapping lines, the correlation between the experimental $\mathrm{H}_{\beta}$ lineshapes at times $\mathrm{A}, \mathrm{B}$, and $\mathrm{C}$ and the theoretical $\mathrm{H}_{\beta}$ curves was not very good.

Figure 3.10 Spectrum acquired at time F of Fig. 3.4.

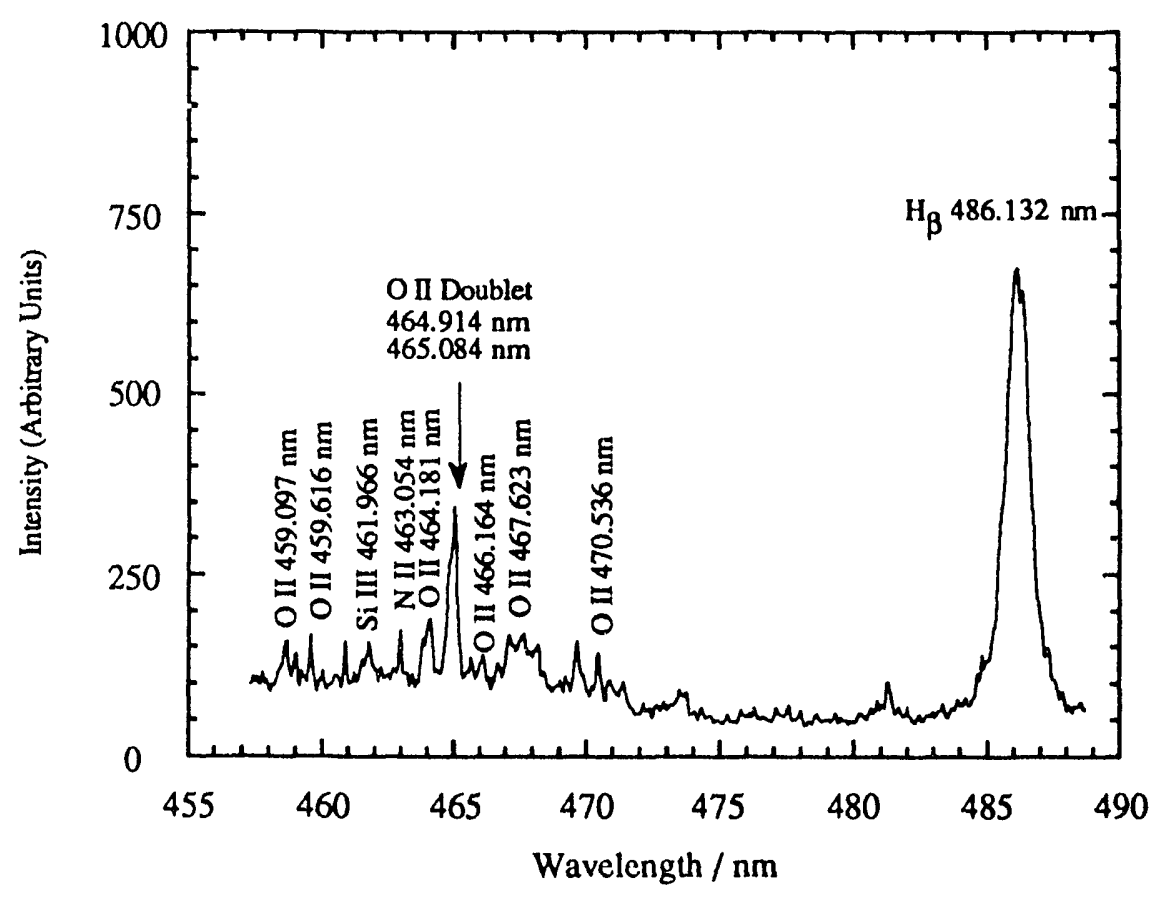

According to Vidal et al..$^{3.6}$, the theoretical $\mathrm{H}_{\beta}$ profiles show too much structure near the peak. In particular, the predicted dip in intensity at the line center is 
much greater than that observed experimentally. To minimize this effect on the comparison between experiment and theory both the experimental and theoretical $\mathrm{H}_{\beta}$ curves have been area normalized. Because the FWHM of the theoretical $\mathrm{H}_{\beta}$ profiles increases with increasing electron density, area normalization causes the peak intensities to decrease and the wing intensities to increase with increasing electron density (see Fig. 3.11). Comparison between the area-normalized experimental and theoretical $\mathrm{H}_{\beta}$ profiles to determine electron density works well when a clean experimental $\mathrm{H}_{\beta}$ signal is obtained. However, if there are interference lines which

Figure 3.11 Illustration of effect of area normalization on theoretical $\mathrm{H}_{\beta}$ profiles

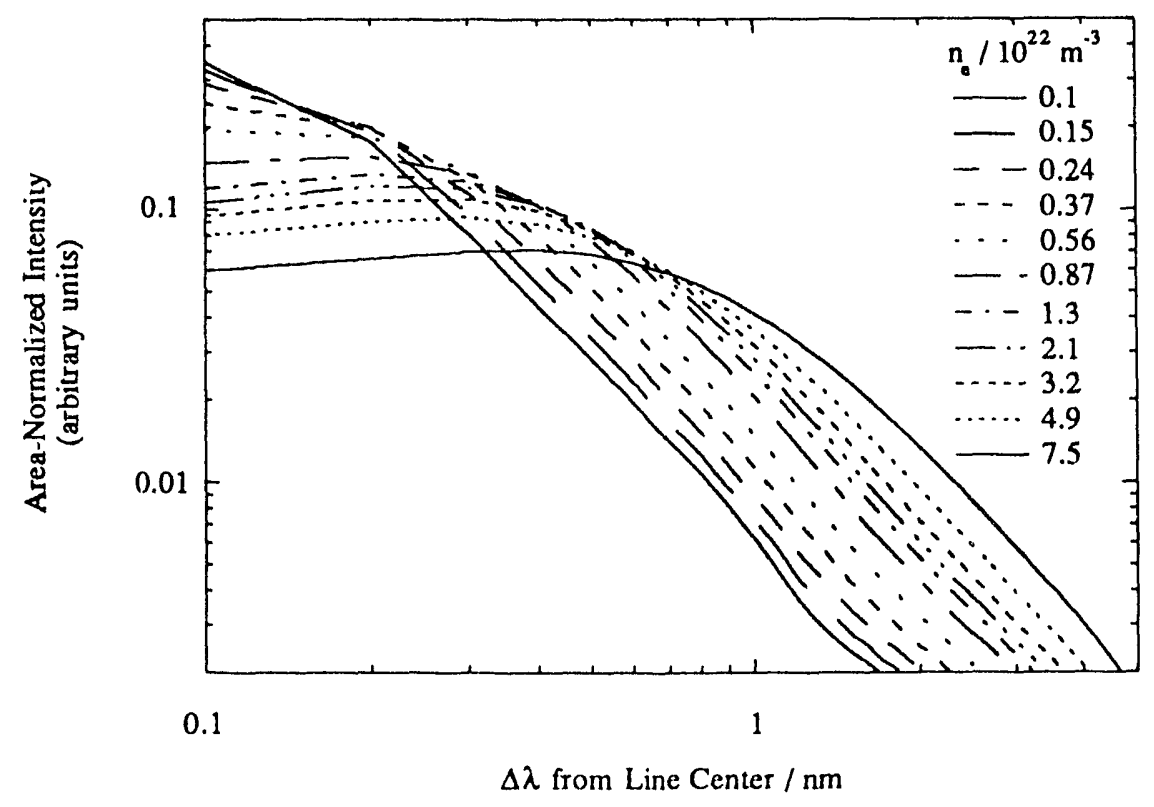


overlap the $\mathrm{H}_{\beta}$ signal the area normalized experimental lines tend to be skewed in shape. The wing intensities tend to be higher and the peak intensity lower than they should. The result is that, when choosing the background spectrum of the experimental $\mathrm{H}_{\beta}$ profile which causes its shape to most nearly coincide with the theory, one underestimates. Ther $r_{0 r e}$ the theoretical $\mathrm{H}_{\beta}$ intensity which most nearly matches the experimental data is undoubtedly too high, and furthermore, it is difficult to estimate by how much.

Figure 3.12 shows the normalized intensity of the experimental $\mathrm{H}_{\beta}$ line obtained at time B, along with some of the theoretical curves. The bold theoretical curve is the one which I judged to most nearly match the experimental data. Because of the problems explained above however, the electron density corresponding to this curve $\left(5.6 \times 10^{22} \mathrm{~m}^{-3}\right)$ is believed to be too high. Despite this problem the electron densities estimated by this method at times A-C were believed to be of the correct order of magnitude. Section 3.10 gives results of electron densities estimated at these positions using a new method of absolute calibration of the photodiode array system which supports this belief.

Figure 3.13 shows the results of using the above-described procedure at time D (similar results were obtained at times E-H). It can be seen that there is a much better correspondence between experiment and theory at this time than at time $\mathrm{B}$. The uncertainty in the choice of the theoretical curves which best matched the experimental data resulted in as estimated electron density uncertainty of $\pm 20 \% .3 .7$ Table 3.2 shows the estimated electron densities. 
Figure 3.12 Experimental and theoretical $\mathrm{H}_{\beta}$ intensities vs distance from center of $H_{\beta}$ line at time $B$ of Fig. 3.4.

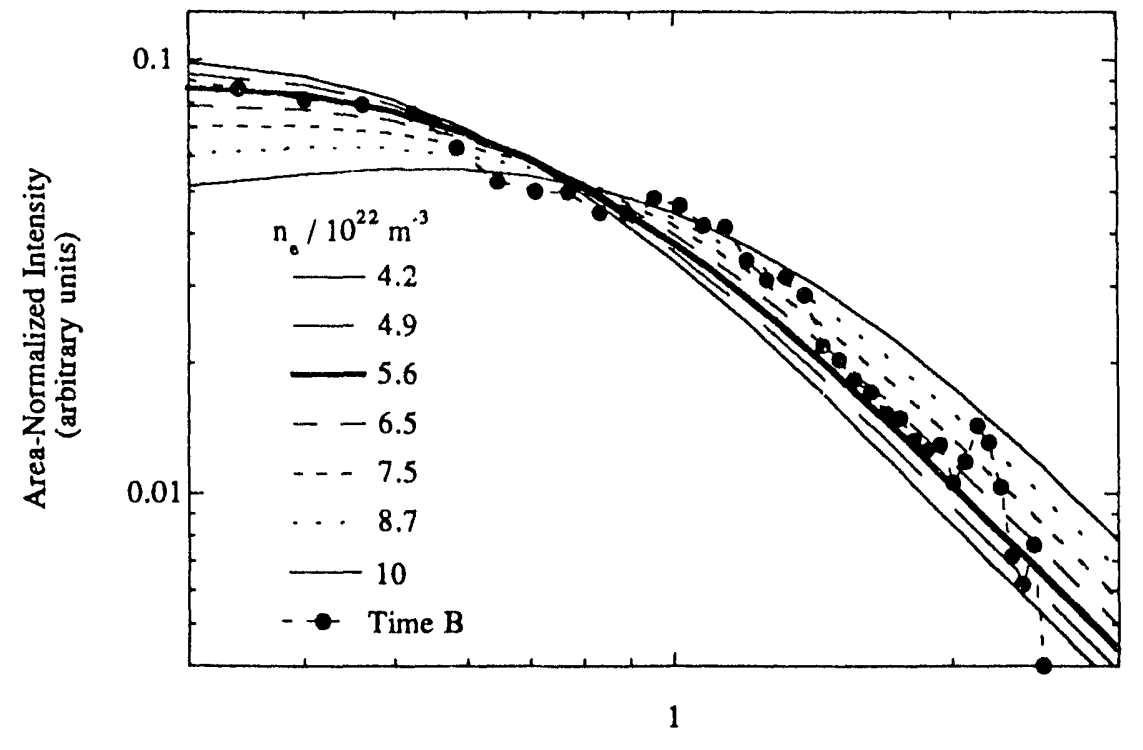

$\Delta \lambda$ from Line "nnter/nm

Figure 3.13 Experimental and theoretical $\mathrm{H}_{\beta}$ intensities vs distance from center of $\mathrm{H}_{\beta}$ line at time D of Fig. 3.4.

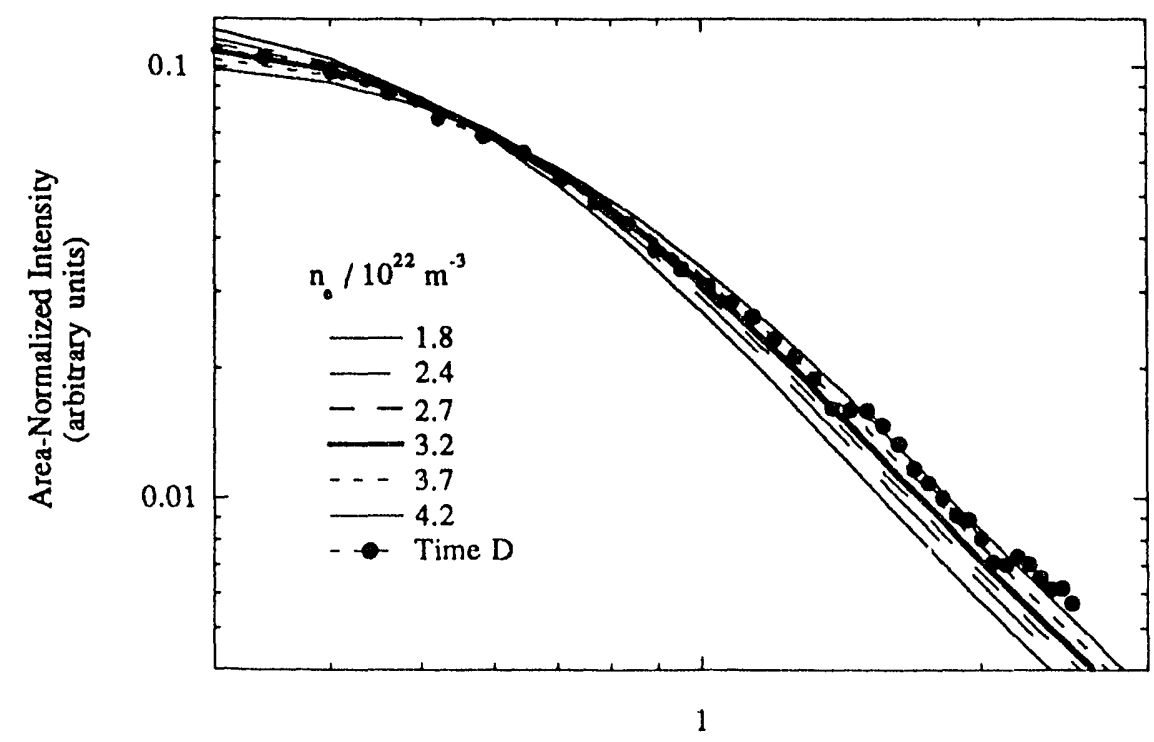

$\Delta \lambda$ from Line Center / nm 
Table 3.2 Electron densities at times shown on Fig. 3.4

\begin{tabular}{cc} 
Time & $\begin{array}{c}\text { Electron density } \\
\left(\mathrm{n}_{\mathrm{e}} / 10^{22} \mathrm{~m}^{-3}\right)\end{array}$ \\
\hline
\end{tabular}

$\begin{array}{cc}\text { A } & 4.9^{*} \\ \text { B } & 5.6^{*} \\ \text { C } & 7.5^{*} \\ \text { D } & 3.2 \\ \text { E } & 0.56 \\ \text { F } & 1.3 \\ \text { G } & 0.87 \\ \text { H } & 0.87\end{array}$

* These electron densities represent the upper bound. The large uncertainties are caused by the presence of impurity lines falling under the $\mathrm{H}_{\beta}$ line.

\subsection{Temperature calculation via Boltzmann Plots}

Temperatures of the plasma armatures were calculated by means of Boltzmann plots. The following is a description of this method of temperature calculation.

The emissive power of an atomic transition can be written as

$$
\varepsilon_{1}=S X=(1 / 4 \pi) A N_{l} h c / \lambda
$$

where $S$ is the total intensity of the transition (directly proportional to the area under the line shape of the transition), $\mathrm{X}$ is an absolute calibration constant of the spectral 
system, $A$ is the transition probability, $N_{l}$ is the number density of the excited atoms, $h$ is Planck's constant, $c$ is the speed of light, and $\lambda$ is the wavelength of the transition. The Boltzmann distribution can then be written to relate the number density of the excited state atoms to the total number density:

$$
N_{1}=N \frac{g}{Z} \exp \left(\frac{-E}{k T}\right)
$$

where $g$ is the degeneracy of the level, $Z$ is the partition function of the species, $E$ is the upper energy level of the transition, $\mathrm{T}$ is the temperature, and $\mathrm{k}$ is the Boltzmann constant. By combining Eqs. 2 and 3 one can write for two transitions of a given species

$$
\frac{S_{1}}{S_{2}}=\frac{A_{1}}{A_{2}} \frac{\lambda_{2}}{\lambda_{1}} \frac{g_{1}}{g} \exp \left(\frac{-\left(E_{1}-E_{2}\right)}{k T}\right)
$$

Letting $U=S \lambda / g A$, Eq. 4 can be rewritten as

$$
\left(\ln U_{1}-\ln U_{2}\right)=T^{-1}\left(E_{2}-E_{1}\right) / k
$$

Still further, if one lets $Y=\ln U$, Eq. 5 can be seen to be the equation of a straight line with slope $\mathrm{T}^{-1}$. A Boltzmann plot is constructed by measuring $S$ for several transitions of a species for which the atomic data is known and plotting a graph with $\mathrm{Y}$ on the ordinate axis and $\mathrm{E} / \mathrm{k}$ on the abscissa. A straight line is fit to this data via a least squares procedure, and the temperature is deduced by measuring its slope.

The above method could be used only to calculate the electron temperatures of the plasma armatures and not the atomic temperatures. The reason for this is that for atomic temperatures to be calculated the plasma must be in local thermodynamic 
equilibrium (LTE) and the measured electron densities were slightly lower than those normally required (typically $\left.6 \times 10^{22} \mathrm{~m}^{-3}\right)^{3.8}$ for LTE to be assumed. However, the assumption of partial local thermodynamic equilibrium (PLTE) in the plasma is sufficient for calculating electron temperatures using Eq. 5, as long as all the excited states considered are in PLTE. The PLTE assumption implies that excited states, created by electron impact, have relative populations determined by the mean kinetic energy of the exciting electrons. Thus the "temperature" describing the relative populations of atoms in excited states determined from a Boltzmann plot is the electron temperature (and is not necessarily equal to the kinetic temperature of the atoms). In the plasmas studied the PLTE assumption implies a common temperature between the electrons and the excited state populations down to $\mathrm{n}=3$ for $\mathrm{O}^{+}$. The $\mathrm{O}^{+}$ transitions are of the type $2 \mathrm{p}^{2} 31-2 \mathrm{p}^{2} 3 \mathrm{~s}$. The transitions at $459.097,464.181$, $466.164,467.623,469.897$, and $470.536 \mathrm{~nm}$ were used to calculate the electron temperature. A Boltzmann plot for the excited states of $\mathrm{O}^{+}$is shown in Fig. 3.14. Each data point represents a single measurement. The solid line on the plot is the best fit of a Boltzmann distribution to the data. From the plot it appears that the PLTE assumption is reasonable. The electron temperature is calculated from the gradient of the fitted line. The estimated error in the inferred temperature is the standard deviation of the gradient. 3.9

The areas under the spectral lines were computed by graphical integration of the data. This restricted us to non-overlapping or slightly overlapping lines because one cannot accurately determine the intensities of overlapped transitions. The estimated error in the area determination is about $\pm 10 \%$. The uncertainty in the area determination is masked in the Boltzmann plot because it appears in the argument of a 
Figure 3.14 Boltzmann plot for $\mathrm{O}^{+}$transistions at time B of Fig. 3.4.

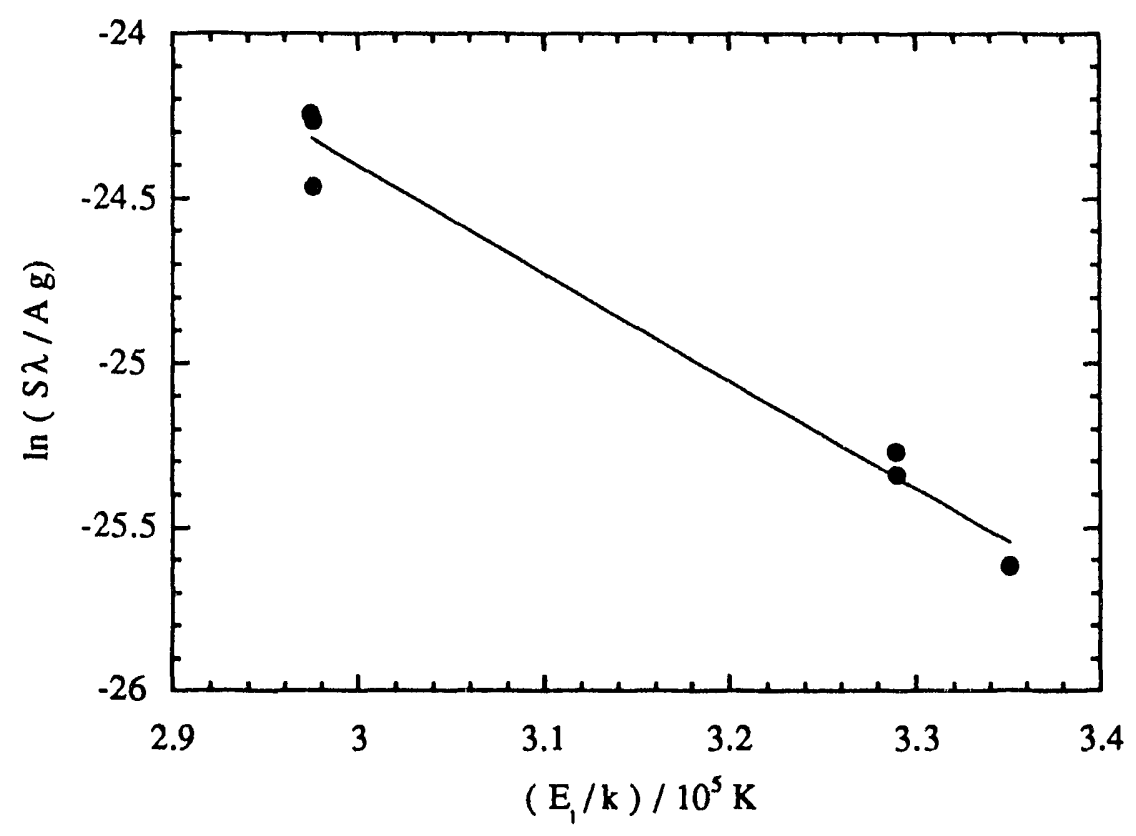

logarithm. Note that the temperature measurements are integrations of the plasma emission along the direction of observation. Non-uniformities in the plasma are averaged to some extent, but the result tends to be weighted towards the peak plasma temperatures and thin boundary layers will have little effect. This point is discussed further in Appendix III.

The electron temperature at times A-D of Fig. 3.4 were estimated using Boltzmann plots. The results of these calculations are shown in Fig. 3.15 labeled Method 1. The times shown on the abscissa are relative to arrival of the leading edge of the first plasma armature at this location. Temperatures are in the range 20000 - 
Figure 3.15 Electron temperature estimates for times indicated on Fig. 3.4.

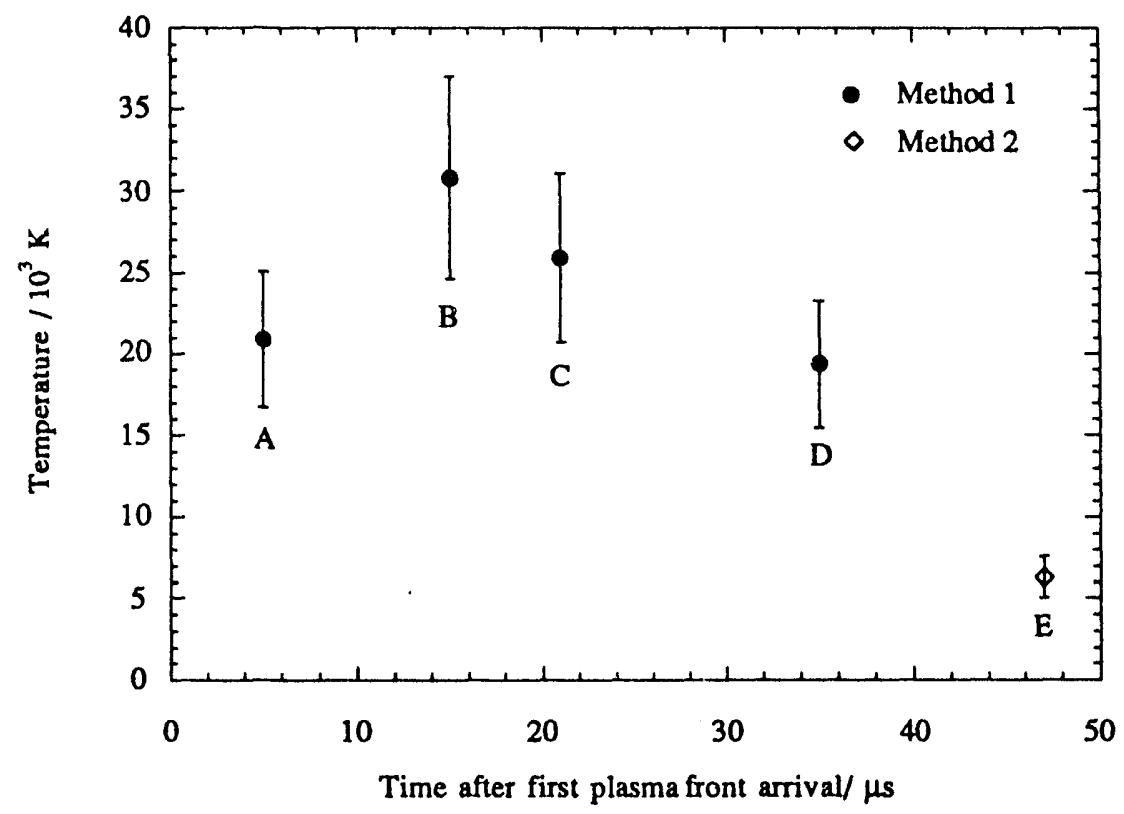

$30000 \mathrm{~K}$, with a maximum estimated error of $\pm 3000 \mathrm{~K}$. Note that the kinetic temperature of the atoms is likely to be lower than the electron temperature estimated from excited state populations because the plasma is not in LTE. This method could not be used for times $\mathrm{E}-\mathrm{H}$ because no significant $\mathrm{O}^{+}$transitions were detected in the spectra taken at these times; the $\mathrm{H}_{\beta}$ transition was the only prominent line in the wavelength interval studied. (Times F-H correspond to spectra from the second armature.) The electron temperatures were estimated at these times using a new absolute optical calibration described in the following section. The temperatures calculated using this method are labeled Method 2 in Fig. 3.15. The data points corresponding to temperatures calculated via Methods 1 and 2 for the same time have 
been slightly displaced from each other to show the estimated errors for each method more clearly.

3.9 In-situ absolute calibration of the optical system

A new method for absolute calibration of the optical system was devised which does not rely on a tungsten strip lamp. This method has advantages over the standard tungsten strip lamp calibration in cases where geometrical constraints make it difficult to compare the flux from the plasma source to the one from the lamp. The absolute calibration was achieved by using the electron temperature determined at time $D$ via the Boltzmann plot and the electron density at time $D$ obtained as explained in section 3.7 .

The underpopulation factor for the upper level of $\mathrm{H}_{\beta}(n=4)$ is equal to 1 at these electron densities and temperatures, 3.10 so the Saha and Boltzmann distributions can be used to relate the total number density of hydrogen with the population of the upper states for the $\mathrm{H}_{\beta}$ transition. From the Saha equation

$$
\frac{n_{i}{ }^{+} n_{e}}{n_{i}}=2 \frac{Z_{\text {ion }}}{Z_{\text {atom }}}\left(\frac{2 \pi m_{\mathrm{e}} T_{e}}{h^{2}}\right)^{3 / 2} \exp \left(-\frac{E-\Delta E}{k T_{e}}\right)
$$

where subscript ' $i$ ' denotes a species, $n_{i}$ is the number of neutral atoms, $n_{i}{ }^{+}$is the number of singly ionized atoms, $\mathrm{Z}$ is the partition function, $\mathrm{h}$ is Planck's constant, $\mathrm{m}_{\mathrm{e}}$ is the mass of an electron, $\mathrm{T}_{\mathrm{e}}$ is the electron temperature, $\mathrm{E}$ is the ionization energy, and $\triangle \mathrm{E}$ is the lowering of ionization energy due to plasma-particle interactions near the ionization limit. 
In general, electrons could be produced by ionization of several species. One can show that the electrons arise primarily from ionized hydrogen by the following calculation. Writing Eq. 6 for $\mathrm{O}^{+}$and $\mathrm{H}^{+}$gives,

$$
\begin{aligned}
& \frac{n_{H^{+}} n_{e}}{n_{H}}=2 \frac{Z_{H^{+}}}{Z_{H}}\left(\frac{2 \pi m_{e} k T_{e}}{h^{2}}\right)^{3 / 2} \exp \left(-\frac{E^{H}-\Delta E^{H}}{k T_{e}}\right), \\
& \frac{n_{O^{+}} n_{e}}{n_{O}}=2 \frac{Z_{O^{+}}}{Z_{O}}\left(\frac{2 \pi m_{e} k T_{e}}{h^{2}}\right)^{3 / 2} \exp \left(-\frac{E^{O}-\Delta E^{O}}{k T_{e}}\right) .
\end{aligned}
$$

Because $\Delta \mathrm{E}$ is a function of the electron density, temperature, and charge of a species only, $\Delta \mathrm{E}^{\mathrm{H}}=\Delta \mathrm{E}^{\mathrm{O}}$. Dividing Eq. 7 by Eq. 8 produces

$$
\frac{n_{H^{+}}}{n_{O^{+}}}=\frac{n_{H}}{n_{O}} \frac{Z_{H^{+}}}{Z_{H}} \frac{Z_{O}}{Z_{O^{+}}} \exp \left(-\frac{E^{H}-E^{O}}{k T_{e}}\right) \text {. }
$$

Now, since $E^{H}=109678.7 \mathrm{~cm}^{-1}$ and $E^{O}=109836.7 \mathrm{~cm}^{-1}$, and assuming that $\mathrm{T}_{\mathrm{e}}=$ $20,000 \mathrm{~K}$, Eq. 9 can be solved as

$$
\frac{\mathrm{n}_{\mathrm{H}^{+}}}{\mathrm{nO}^{+}}=\frac{\mathrm{n}_{\mathrm{H}}}{\mathrm{n}_{\mathrm{O}}} \frac{1}{2.6} \frac{12.9}{5.83}(1.01)=0.86 \frac{\mathrm{n}_{\mathrm{H}}}{\mathrm{n}_{\mathrm{O}}}
$$

Because no neutral $\mathrm{O}$ transitions were observed and since neutral $\mathrm{H}$ transitions are prominent in the plasma, e.g. the $\mathrm{H}_{\beta}$ transition, it is concluded that $\mathrm{n}_{\mathrm{H}} \gg \mathrm{n}_{\mathrm{O}}$. Hence, from Eq. $7, \mathrm{n}_{\mathrm{H}}{ }^{+}>>\mathrm{nO}^{+}$. From the charge neutrality equation for the plasma one obtains

$$
\mathrm{n}_{\mathrm{e}}=\mathrm{n}_{\mathrm{H}^{+}}+\mathrm{n}_{\mathrm{O}^{+}}^{+}+2 \mathrm{n}_{\mathrm{Si}^{2+}}{ }^{2+}
$$

No lines of $\mathrm{Si}^{+}$were detected in the spectra, from which it was concluded that any silicon present was doubly ionized. The $\mathrm{Si}^{2+}$ and $\mathrm{O}^{+}$were produced from $\mathrm{SiO}_{2}$ in the glass side-walls; the contribution of residual $\mathrm{O}_{2}$ in the vacuum chamber was 
determined to be negligible. Hence, $\mathrm{n}_{\mathrm{H}}{ }^{+} \gg \mathrm{nO}^{+}$implies that $\mathrm{n}_{\mathrm{H}}{ }^{+}>\mathrm{n}_{\mathrm{Si}^{2+}}{ }^{2+}$, and hence Eq. 8 gives $n_{e} \approx n_{H}{ }^{+}$. Equation 4 now becomes

$$
\frac{n_{e}^{2}}{n_{H}}=2 \frac{Z_{H^{+}}}{Z_{H}}\left(\frac{2 \pi m_{e} k T_{e}}{h^{2}}\right)^{3 / 2} \exp \left(-\frac{E^{H}-\Delta E^{H}}{k T_{e}}\right)
$$

If $\mathrm{X}$ is the absolute calibration constant of the system and $\mathrm{S}$ is the total intensity of a transition observed in counts,

$$
\varepsilon_{l}=X S=(1 / 4 \pi) h \vee A n_{l}
$$

where $\varepsilon_{l}$ is the emissive power of the transition, $v$ is the frequency of the transition, and $\mathrm{n}_{l}$ is the number density of atoms in the $l$ th state. Also, the Boltzmann distribution gives

$$
n_{l}=\left(g_{l} / Z\right) n \exp \left(-E_{l} / k T\right)
$$

where $\mathrm{n}$ is the number of atoms in the ground state. Eqs. $12-14$ can be used to calculate $\mathrm{X}$.

The absolute calibration constant $\mathrm{X}$ was calculated only at time $\mathrm{D}$ because only at this time were both the electron temperature calculated using a Boltzmann plot and the graphically-obtained electron density known reliably. By this procedure the value of $X$ was estimated to be $235 \pm 50 \mathrm{~W} / \mathrm{m}^{3}$-count-sr-nm. The uncertainty in $X$ reflects the uncertainties in the electron temperature and density estimates at time D. 
3.10 Electron Temperature and Density Calculations by Absolute Calibration

With the absolute calibration constant known, Eqs. $12-14$ can be used to calculate either the electron temperature for a given value of electron density or vice versa. At times E-H the electron densities were known but not temperatures, so at these times the temperatures were calculated. These temperatures are labelled Method 2 in the Figs. 3.15 and 3.16 for the first and second armatures respectively. At times A-C the electron densities had been estimated using the graphical method but were thought to be too high. Figure 3.17 illustrates the difference between the electron densities estimated using the graphical procedure and those calculated using the

Figure 3.16 Electron temperature estimates for times indicated on Fig. 3.4.

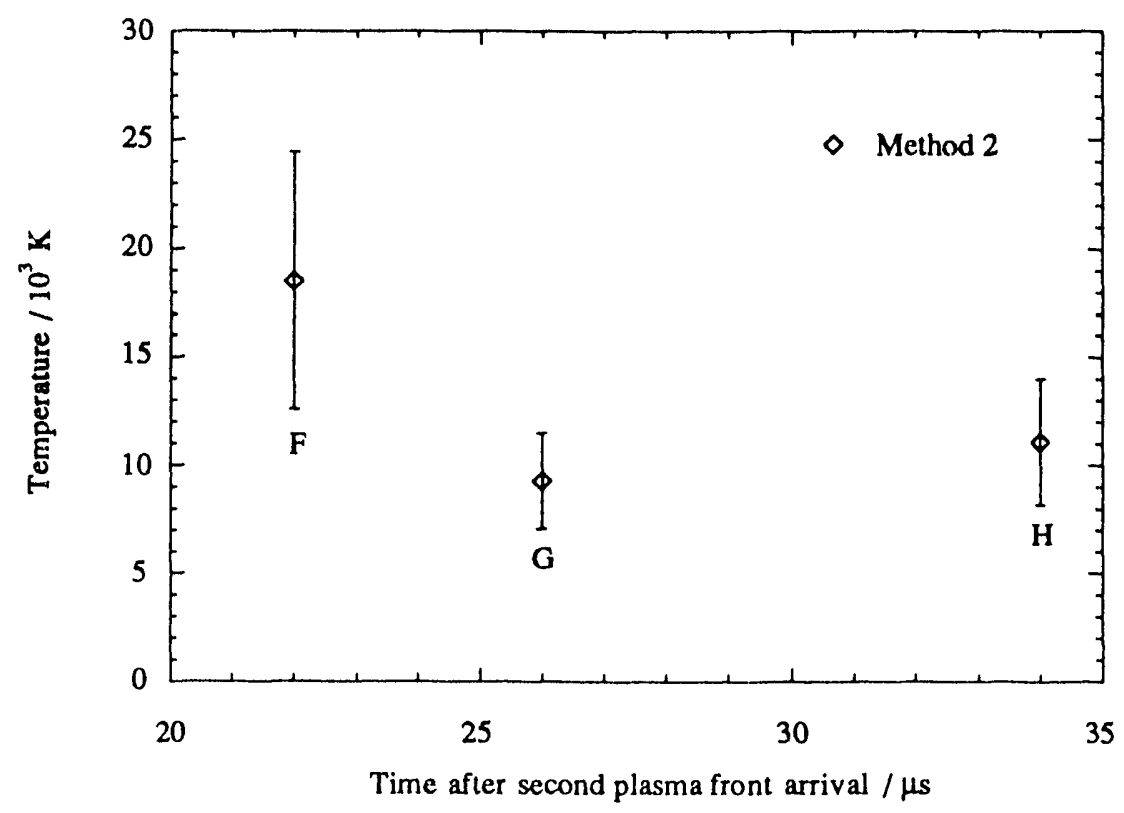


Figure 3.17 Electron densities for Times A-C of Fig. 3.4 estimated using the graphical and absolute calibration methods.

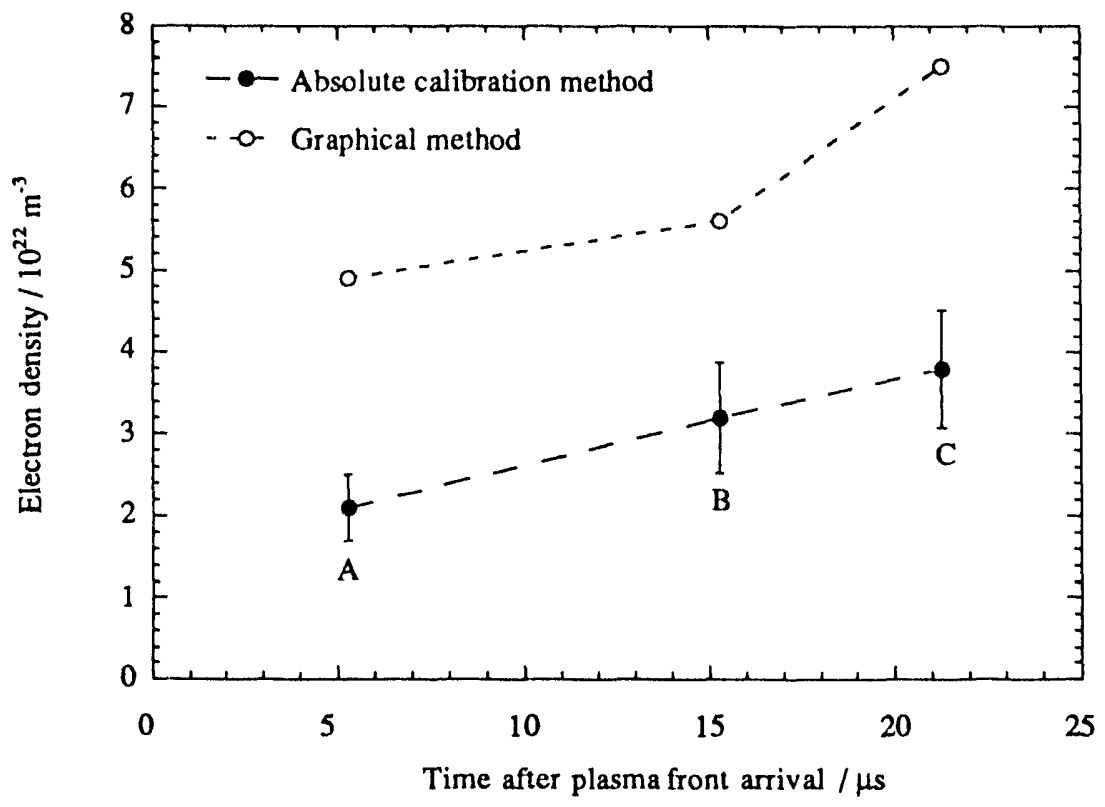

absolute calibration constant. The latter electron density estimates are probably low because oxygen ionization is not accounted for in them. The estimated errors shown in Figs. 3.15, 3.16, and 3.17 were obtained using the method outlined by Bevington. 3.8 Please see Appendix IV for details of this error analysis. 


\section{CHAPTER 4}

\section{TARGET POSITION MEASUREMENTS}

\subsection{Motivation}

The original intent of this project was to study the effects of railgun-generated hydrogen plasmas on targets composed of materials such as stainless steel, graphite, an aluminum. Therefore, the next step undertaken was the characterization of the plasma at the position at which a target would be placed.

\subsection{Equipment Modifications}

Most of the equipment described in Chapter 2 was used in this set of experiments. Only equipment that was modified between the measurements discussed in Chapter 3 and those discussed in this chapter will be discussed here.

The primary equipment modification made was the replacement of the valve used to inject hydrogen into the breech of the railgun. The new valve (hereafter referred to as the Sandia valve when necessary for clarity) was obtained from Dr. Richard Spielman of Sandia National Laboratories, Albuquerque, New Mexico. This valve was solenoid-driven, however, it was of much sturdier construction than the original two valves (see Fig. 2.7 for a schematic diagram of its design). The valve was driven by a variable-voltage power supply (the valve power supply was manufactured by Slapshot Inc., and will be referred to as the "Slapshot driver" hereafter). The variability in the Slapshot driver voltage allowed the speed of the 
valve actuation to be adjusted. At the suggestion of Dr. Spielman the charging voltage was set at $60 \%$ of its maximum. According to Dr. Spielman, experimenters at Sandia have had the greatest success using this charging voltage.

Only slight modifications to the existing hardware were necessary to use the Sandia valve. A BNC vacuum feedthrough with an isolated ground was constructed to allow voltage from its voltage supply to be fed into the vacuum chamber and to the valve solenoid. The valve mounting mechanism (see Fig. 2.7 ) allowed for this valve to be attached to the housing designed for the piezoelectric valve with only slight modification to the housing.

The performance of this valve was studied using the railgun mockup and fast ionization gauge system (FIG) described in Chapter 2. It was found that approximately $3.5 \mathrm{~ms}$ elapsed between the time the trigger was sent to the Slapshot driver and the occurrence of the peak output of the FIG. This measurement was made to determine the timing sequence of the rest of the experiment, in particular, when the capacitor-bank discharge-ignitron needed to be fired.

\subsection{Experimental Procedure Modifications}

\subsubsection{Mode of operation of the railgun}

The primary change in the experimental procedure between the Chapter 3 experiments and the experiments described here was in the firing of the railgun. After conducting the hydrogen pressure experiment (see Section 4.4) it was felt that a 3.5 ms delay between the triggering of the Slapshot driver and discharge of the capacitor bank would allow for consistent firing of the railgun. However, no railgun discharge 
occurred with this delay. Subsequently, the delay was varied, starting at $500 \mu$ s and incrementing by $500 \mu \mathrm{s}$, until the entire range of possible delays from 0 to $9999 \mu \mathrm{s}$ was covered, and at none of these delays did a railgun discharge occur. As a means to find out if the gun would fire at all with the Sandia valve another mode of railgun operation was tried. This mode of operation entailed shorting the discharge ignitron out of the power delivery circuit. Thus, when the capacitor bank was charged the voltage was being applied directly to the railgun rails. In this mode the gun discharge occurred upon introduction of gas from the gas valve. This mode of operation will be referred to as the "passive mode" for the remainder of this work.

The railgun was found to operate reliably using the passive mode of discharge. It was found however, that the timing of the railgun discharge decreased with each successive shot, until a steady delay was reached between the trigger pulse to the Sandia gas valve driver and discharge of the railgun. This delay was found to be about $3.5 \mathrm{~ms}$, as originally expected. When this steady delay mode of operation was reached, use of the discharge ignitron was again tried (called the "active mode" hereafter). The gun did indeed fire after the steady delay was reached. However, the phototransistor data collected using the active mode of discharge were found to be much noisier than that collected in the passive mode. This additional noise apparently originated from the discharge ignitron. Since there was no other benefit to operating in the active mode and better data could be obtained in the passive mode, the passive mode of operation was chosen for the remainder of the experiments described here. Figure 4.1 is a schematic diagram of the experimental setup with the new equipment and passive operation mode depicted. 
Figure 4.1 Experimental Apparatus

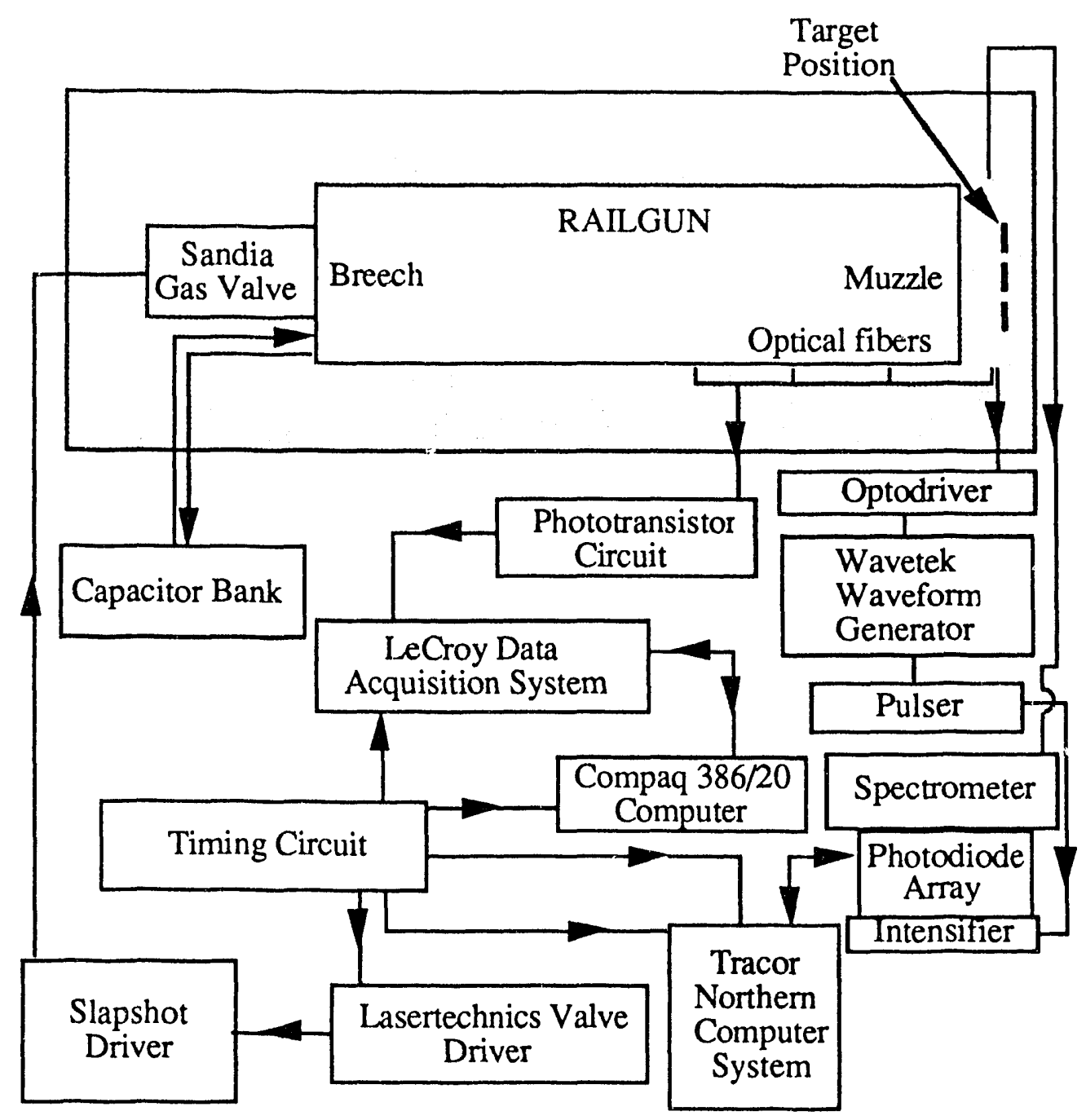




\subsubsection{Triggering of spectral acquisition system}

Even after the valve operation settled into its relatively steady operation state there was still enough variability in the time of discharge after injection of the hydrogen gas to prevent accurate timing of the spectral acquisition system as described in Chapter 3. Therefore, a circuit was designed which could accept a light input as a trigger source and which could output a TTL-level step function to serve as a trigger for the spectral acquisition system (see Fig. 4.2 for a schematic diagram of this circuit, called the "Optodriver" hereafter). An optical fiber of the same type as was used to transmit light to the phototransistors and spectral acquisition system was used to provide the light signal to this circuit. The origin of this light source was the railgun plasma itself, which allowed the delay to the spectral acquisition system to be determined independently of any jitter in the railgun discharge. Figure 4.3 is a timing diagram depicting the sequencing of these experiments.

\subsubsection{Repetition of spectral acquisition experiments}

The Chapter 3 results were obtained by making a single spectral measurement at each of the times shown in Fig. 3.1. In order to improve on the Chapter 3 results, the spectral measurements were conducted at least three times at each position in the plasma armature and the results averaged.

\subsection{Results of Target Position Measurements}

Using the new equipment and mode of operation it was now possible to characterize the plasma armatures at the target position. 
Figure 4.2 Optodriver Circuit

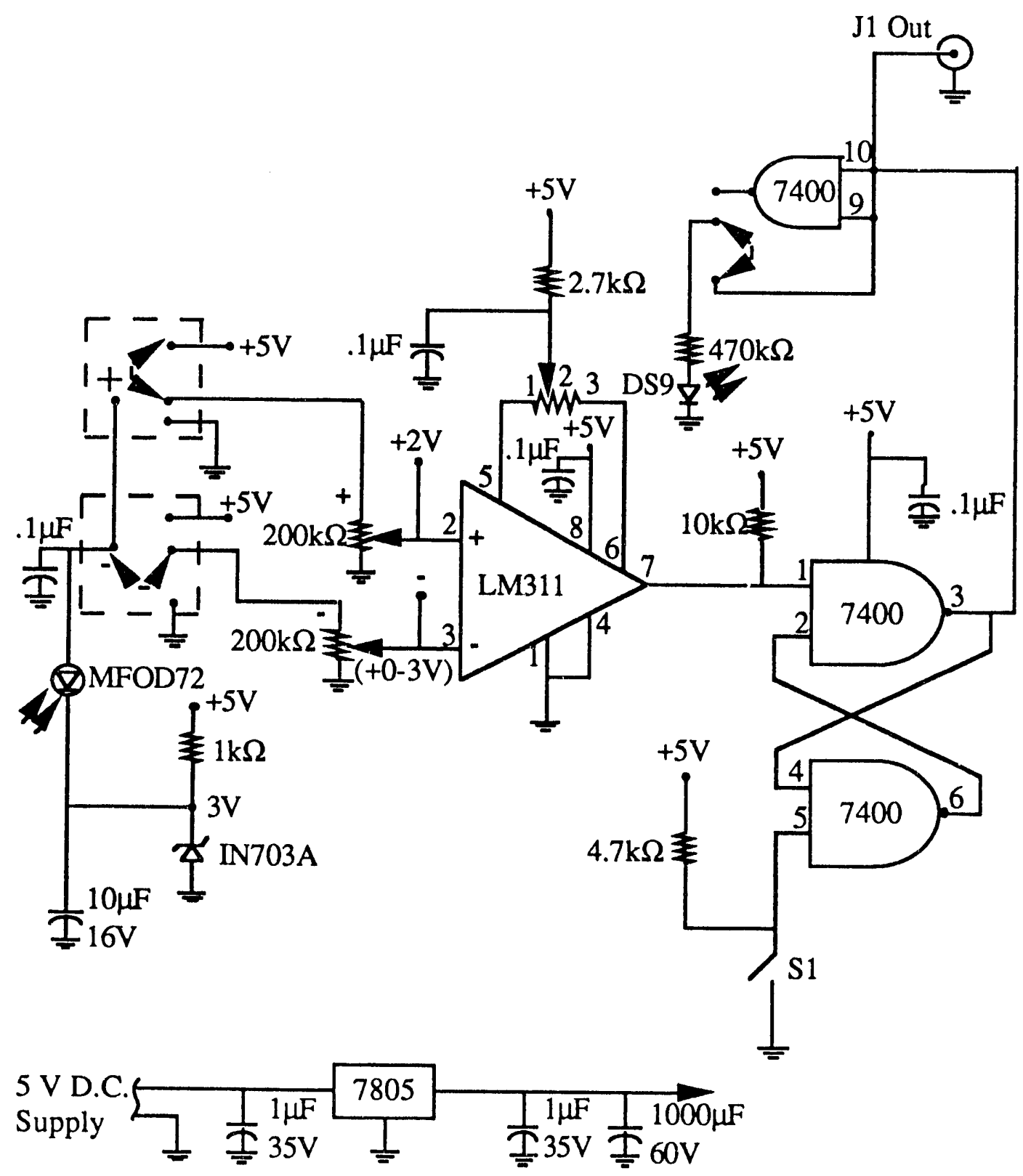


Figure 4.3 Experimental Timing Sequence

TD

Pulse to gas valve

TB Data acquisition system trigger

Optical output from railgun discharge

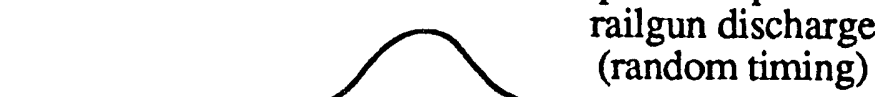

Optodriver pulse sent to

Waveform generator
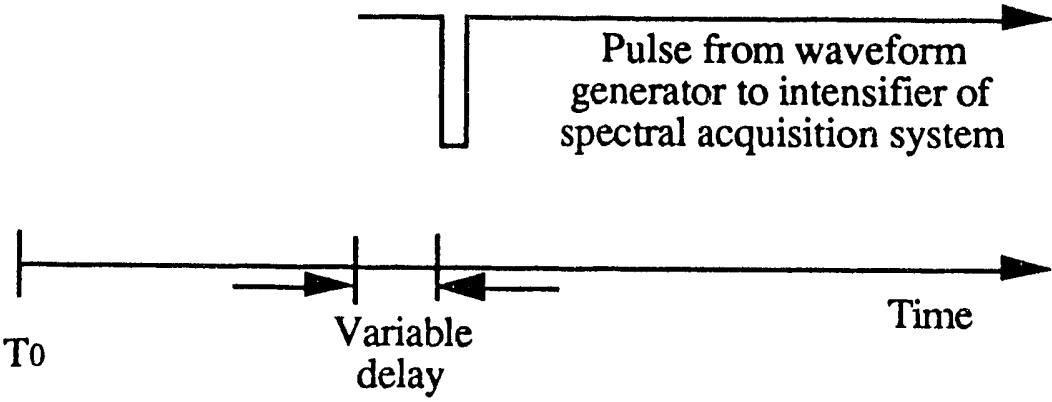


\subsubsection{Velocity measurements}

The Chapter 3 velocity measurements were made using both phototransistor outputs and $\stackrel{\circ}{\mathrm{B}}$-probe outputs to fix the time of arrival of the plasma front at successive points along the railgun bore. Because of the unusual shape of the position versus time data obtained on those experiments only the average velocity of the plasma armatures could be obtained (no curve was found which could accurately fit this data set). Because of this problem, the velocity of the plasma armatures upon arrival at the target position was not known. To overcome this problem the four phototransistor input fibers were positioned starting $457 \mathrm{~mm}$ (18 inches) before the railgun muzzle and spaced at $152 \mathrm{~mm}$ (6 inch) intervals. See Fig. 4.4 for a schematic

Figure 4.4 Optical fiber positions.

Distances shown are in meters.

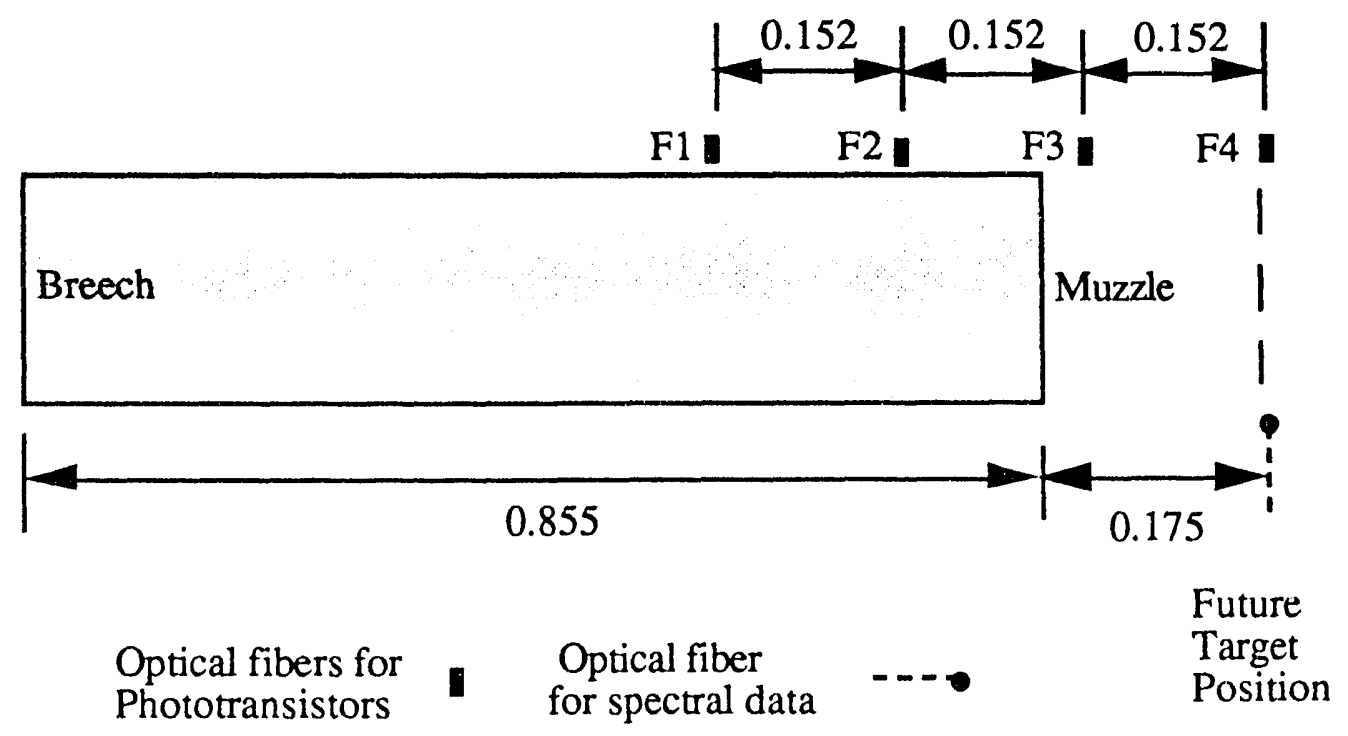


diagram of the phototransistor fiber optic placement. It was not useful to include the $\stackrel{\circ}{\mathrm{B}}$-probes in these measurements because of the limit in the speed at which data could be acquired. The maximum speed of the data acquisition system is limited to $1 \mathrm{MHz}$ for up to eight probes, but could be increased to $2 \mathrm{MHz}$ if only four inputs were used. For a plasma velocity of $150 \mathrm{~km} / \mathrm{s}$ and a probe spacing of $150 \mathrm{~mm}$, only $1 \mu \mathrm{s}$ would elapse between the time of arrival of the plasma front at two successive probes. Operation of the acquisition system at $2 \mathrm{MHz}$ was therefore deemed necessary to obtain meaningful velocity data, which in turn ruled out the use of more than four probes. Since the phototransistors were less susceptible to noise than the

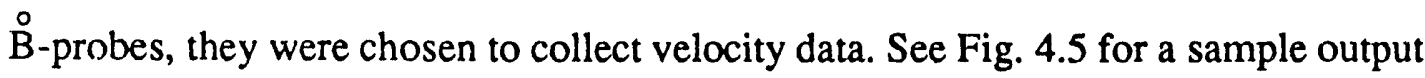
from F4.

Figure 4.6 shows the position of the leading edge of the primary plasma armature versus time. Time "zero" represents the arrival of the plasma armature at position F1. Each data point is an average time of arrival of the plasma armature at that position for seven railgun shots. The error bars represent the average deviation from the mean arrival time at each position for the seven shots. Since arrival of the plasma fronts at positions F2, F3, and F4 were measured relative to the arrival time at the first position F1, the error bar at position F1 was taken as the limit of resolution of the data acquisition system. Also shown on Fig. 4.7 is the polynomial curve fit used to determine the velocity of the plasma armature as a function of time. This curve was obtained using a least-squares fitting procedure provided in the Kaleidagraph ${ }^{\mathrm{TM}}$ software package. The curve shown uses the function 
Figure 4.5 Output of phototransistor F4 vs Time ( $\mathrm{T}_{\mathrm{O}}$ at beginning of output).

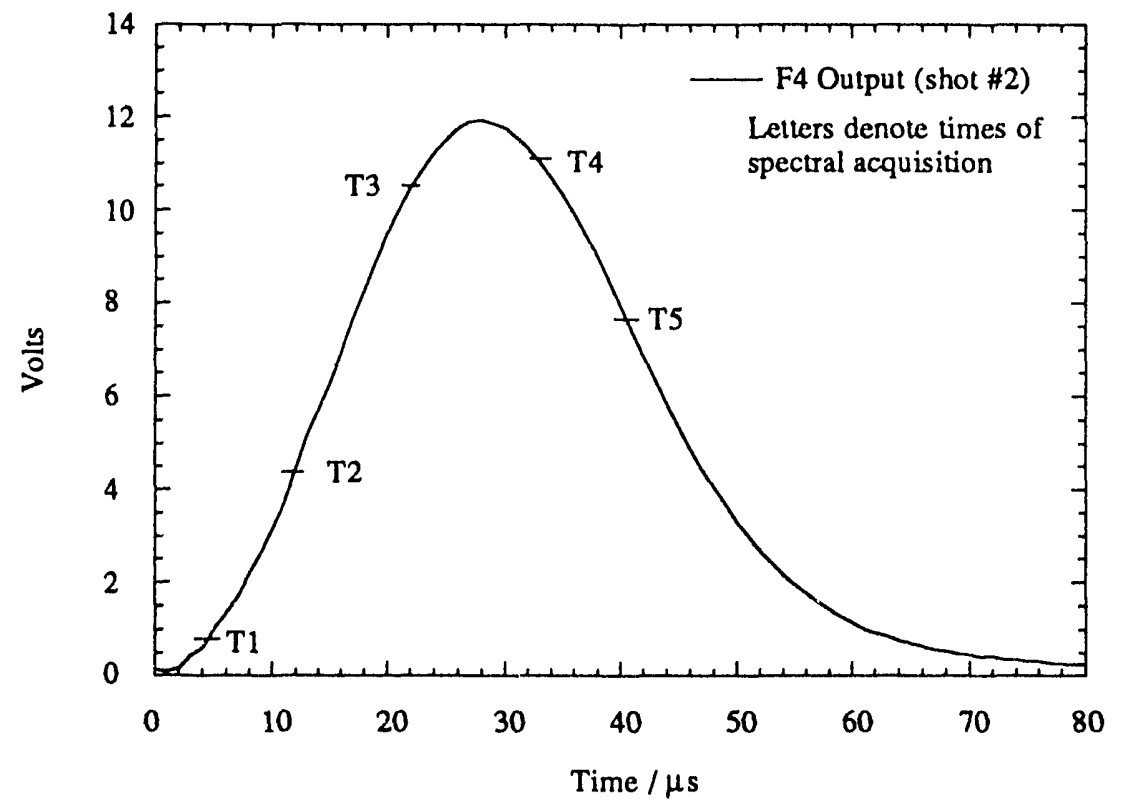

Figure 4.6 Position of the plasma front vs Time (Time 0 represents arrival of the plasma front at phototransitor 1 ).

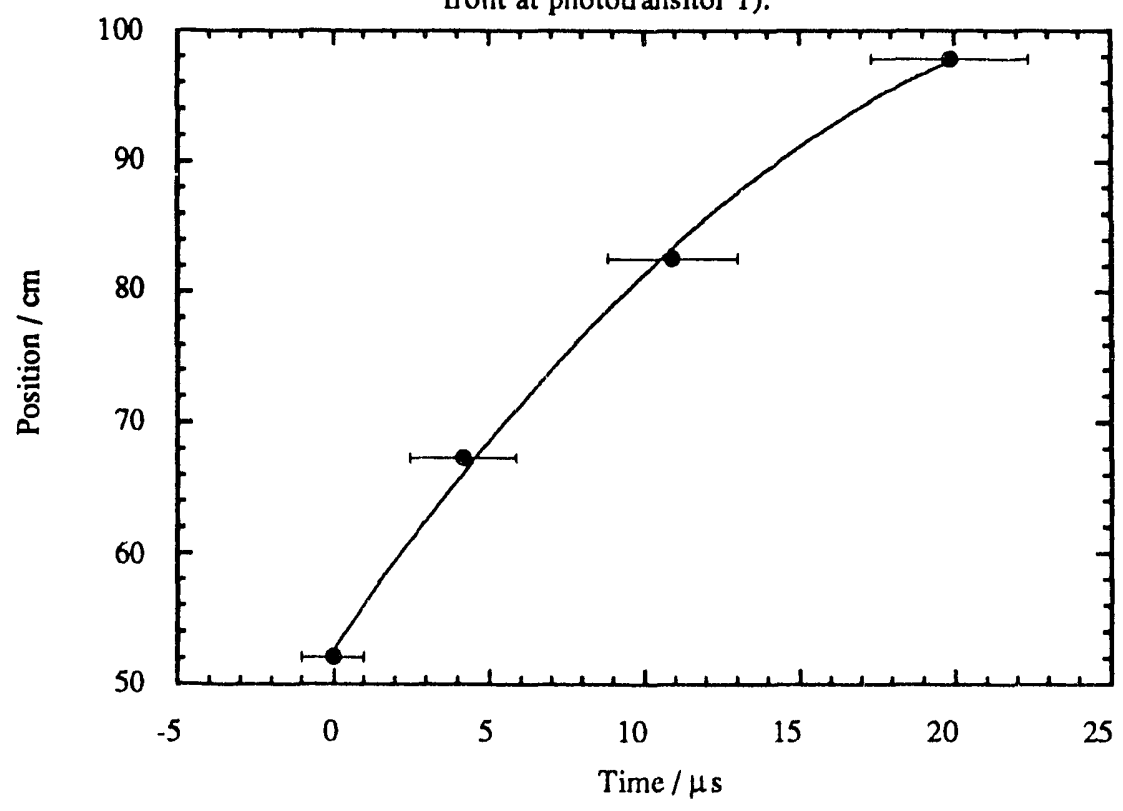




$$
x=52.6354+3.4784 t-0.0611867 t^{2}
$$

where $\mathrm{x}$ is position and $\mathrm{t}$ is time. This function was differentiated to obtain the velocity as a function of position (see Fig. 4.7). From Fig. 4.7 it can be seen that the plasma armature velocity decreased to about $10 \mathrm{~km} / \mathrm{s}$ by the time it reached the target position ( $123 \mathrm{~mm}$ beyond the railgun muzzle).

Figure 4.7 Velocity of the plasma armature vs distance from the railgun breech.

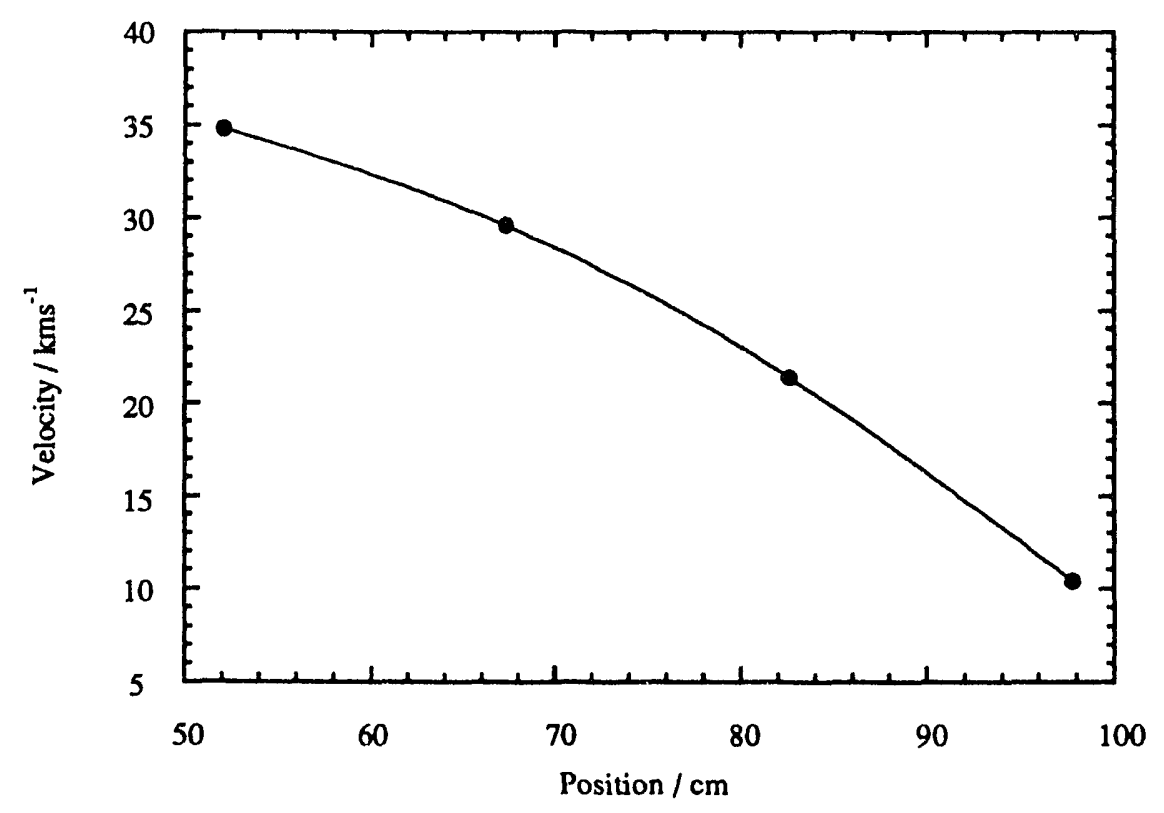

4.4.2 Spectral acquisition and plasma armature composition.

At least three spectra were taken at each of the times shown on Fig. 4.5. This was done because there was shot-to-shot variation in the amplitude of the $\mathrm{H}_{\beta}$ line of about $10 \%$. As in Chapter 3 the highest level of impurities in the hydrogen plasma 
occurred near the front of the plasma armature. In fact, only at time $\mathrm{T} 1$ were there any significant impurity lines present in the spectra which suggests that most of the impurities from the glass sidewalls were released into the hydrogen plasma during the initial breakdown. Figures 4.8 and 4.9 are sample of the spectra taken at the target position at Times T1 and T2 respectively. Comparing Fig. 4.8 with Fig. 3.2 one can see that the present set of experiments yielded much purer $\mathrm{H}_{\beta}$ spectra. From the standpoint that we were originally trying to produce hydrogen plasma, this increase in purity was a highly desirable characteristic of the new valve and mode of operation of the railgun. A drawback, however, was that the electron temperatures could not be calculated as accurately as in the Chapter 3 experiments due to the smaller number of $\mathrm{O} I I$ lines.

Figure 4.8 Spectrum obtained at Time T1 of Fig. 4.5

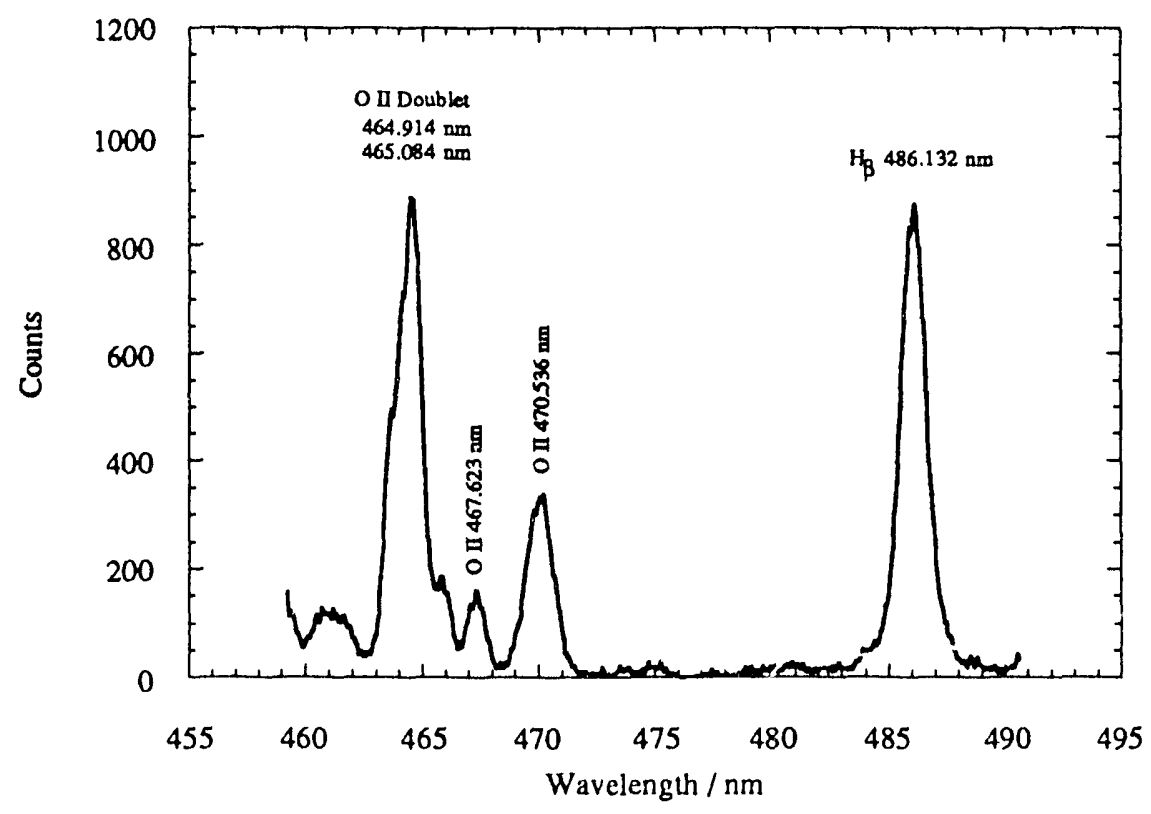


Figure 4.9 Spectrum obtained at Time T2 of Fig. 4.5

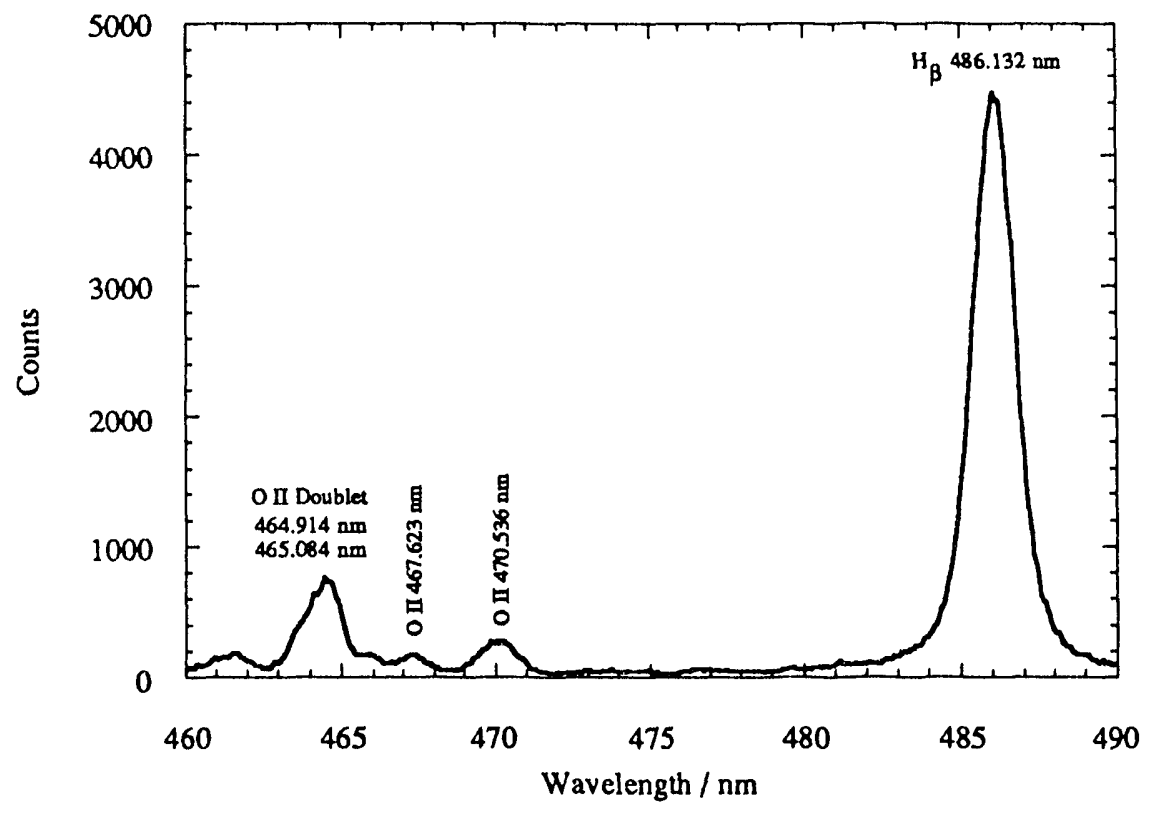

\subsubsection{Electron density measurements}

Electron density measurements were performed following the method detailed in Appendix IV. Because of the high purity of the spectra acquired in this set of experiments the $\mathrm{H}_{\beta}$ profiles were found to match the theoretical profiles more closely See Fig. 4.10 for an example of the profile obtained at time T3. Table 4.1 shows the mean electron density at each time indicated on Fig. 4.5. The uncertainty in the choice of the correct theoretical curves resulted in an estimated uncertainty of $\pm 20 \%$ in the electron densities. 4.1 
Figure 4.10 Experimental $\mathrm{H}_{3}$ profile obtained at Time T3 of Fig. 4.5 and theoretical $H_{\beta}$ profiles.

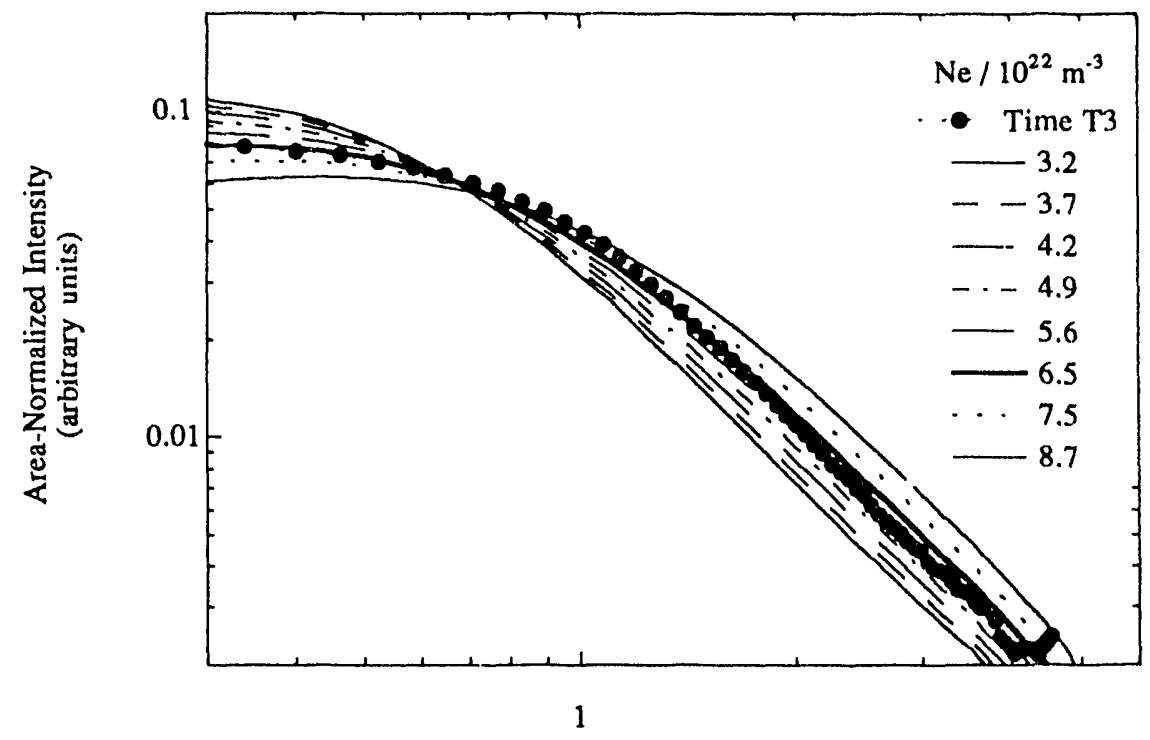

$\Delta \lambda$ from Line Center $/ \mathrm{nm}$

Table 4.1 Mean electron densities at times shown in Fig. 4.5

\begin{tabular}{cr} 
Time & $\begin{array}{r}\text { Electron } \\
\left(/ 10^{22} \mathrm{~m}\right.\end{array}$ \\
\hline $\mathrm{T} 1$ & 2.1 \\
$\mathrm{~T} 2$ & 4.1 \\
$\mathrm{~T} 3$ & 5.8 \\
$\mathrm{~T} 4$ & 4.2 \\
$\mathrm{~T} 5$ & 1.6
\end{tabular}




\subsubsection{Temperature measurements via Boltzmann plots}

At times T1 and T2 of Fig. 4.5 distinct OII lines were identified at $467.6 \mathrm{~nm}$ and $470.5 \mathrm{~nm}$ as well as the doublet at 464.9 and $465.1 \mathrm{~nm}$. To obtain Boltzmann plot coordinates for the doublet it was necessary to use the following equation:

$$
\frac{S_{1}+S_{2}}{S_{3}}=\frac{\frac{A_{1} g_{1}}{\lambda_{1}} \exp \left(\frac{-E_{1}}{k T_{e}}\right)+\frac{A_{2} g_{2}}{\lambda_{2}} \exp \left(\frac{-E_{2}}{k T_{e}}\right)}{\frac{A_{3} g_{3}}{\lambda_{3}} \exp \left(\frac{-E_{3}}{k T_{e}}\right)}
$$

where $S$ is the area under the transition, $A$ is the transition probability, $\lambda$ is the wavelength, $g$ is the degeneracy, $E$ is the energy level of the transition, $k$ is Planck's constant, and $T_{e}$ is the electron temperature. Because the energy levels of the doublet were nearly the same (207003 and $206731 \mathrm{~cm}^{-1}$ for the transitions at 464.9 and $465.1 \mathrm{~nm}$ respectively), $E_{1}$ and $E_{2}$ of Eq. 1 were replaced by their mean value. Use of this mean value caused the Boltzmann plot abscissa coordinate $\mathrm{E} / \mathrm{k}$ to be in error by only $0.07 \%$, a negligible amount. The ordinate value for the doublet was given by

$$
y=\ln \left(\frac{S_{1}+S_{2}}{\frac{A_{1} g_{1}}{\lambda_{1}}+\frac{A_{2} g_{2}}{\lambda_{2}}}\right)
$$

The other two points on the plot were determined using the coordinates $E / \mathrm{k}$ and $\ln \left(\frac{\mathrm{S} \lambda}{\mathrm{Ag}}\right)$. Figure 4.11 shows the Boltzmann plot from the first of four spectra obtained at time $\mathrm{T} 1$ of Fig. 4.5. Temperatures were again obtained from the slopes of the Boltzmann plots. Table 4.2 lists the temperatures calculated using Eq. 2 for each of the spectra taken at times T1 and T2 of Fig. 4.5. 
Figure 4.11 Boltzmann plot for $\mathrm{O}^{+}$transistions at time $\mathrm{Tl}$ of Fig. 4.5.

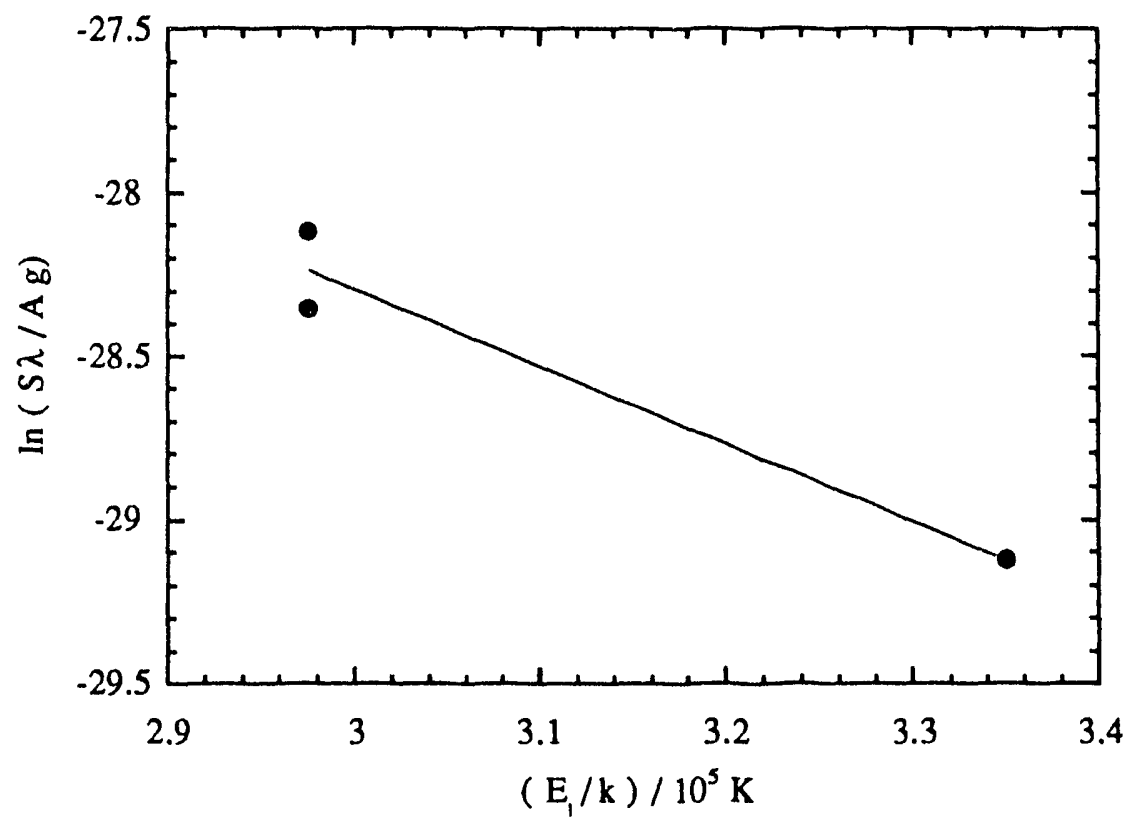

Table 4.2 Boltzmann temperatures for times T1 and T2 of Fig. 4.5.

\begin{tabular}{ccc} 
Time & $\begin{array}{c}\text { Spectrum } \\
\text { Number }\end{array}$ & $\begin{array}{c}\text { Temperature } \\
\left(/ 10^{3} \mathrm{~K}\right)\end{array}$ \\
\hline $\mathrm{T} 1$ & 1 & 42.3 \\
& 2 & 35.7 \\
& 3 & 36.3 \\
$\mathrm{~T} 2$ & 4 & 35.6 \\
& 1 & 33.8 \\
& 2 & 34.3 \\
& 3 & 34.6
\end{tabular}


The estimated error in these temperatures is the standard deviation of the gradient. ${ }^{3.9}$ Using the largest percentage error in the above temperatures, the mean temperatures were found to be $37.4 \pm 9.4 \times 10^{3} \mathrm{~K}$ and $34.2 \pm 8.5 \times 10^{3} \mathrm{~K}$ at times $\mathrm{T} 1$ and $\mathrm{T} 2$ respectively.

\subsubsection{In-situ absolute calibration constant calculations}

Because the electron temperatures and densities were known for each of the spectra taken at times $\mathrm{T} 1$ and $\mathrm{T} 2$ the in-situ absolute $\mathrm{r}$ alibration constant $\mathrm{X}$ discussed in Chapter 3 could be calculated for each one. The mean value of $\mathrm{X}$ was found to be $69.6 \mathrm{~W} / \mathrm{m}^{3}$-count-sr-nm with an estimated error of $\pm 11.7 \mathrm{~W} / \mathrm{m}^{3}$-count-sr-nm. Note that this value of $X$ is different from that calculated in Chapter 3 of $X=235 \mathrm{~W} / \mathrm{m}^{3}$. count-sr-nm. This discrepancy resulted from the difference in the intensifier gain settings used for the two sets of experiments and the change in alignment of the focussing optics.

\subsubsection{In-situ temperature calculations}

Using the above in-situ absolute calibration constant and the electron density for each of the spectra taken, average electron temperatures were calculated at each of the times shown in Fig 4.4. Figure 4.12 shows these temperatures at each time, along with the Boltzmann temperatures computed for times $\mathrm{T} 1$ and T2. The uncertainties in the temperatures were estimated following the method outlined in Appendix IV. The equation used to estimate these uncertainties was

$$
\sigma_{\mathrm{Te}}=\frac{2 \mathrm{~T}_{\mathrm{e}}}{3}\left(4 \frac{\sigma^{2} \mathrm{n}_{\mathrm{e}}}{\mathrm{n}_{\mathrm{e}}^{2}}+\frac{\sigma^{2} \mathrm{~A}}{\mathrm{~A}^{2}}+\frac{\sigma^{2} \mathrm{~S}}{\mathrm{~S}^{2}}+\frac{\sigma^{2} \mathrm{X}}{\mathrm{X}^{2}}\right)^{1 / 2},
$$




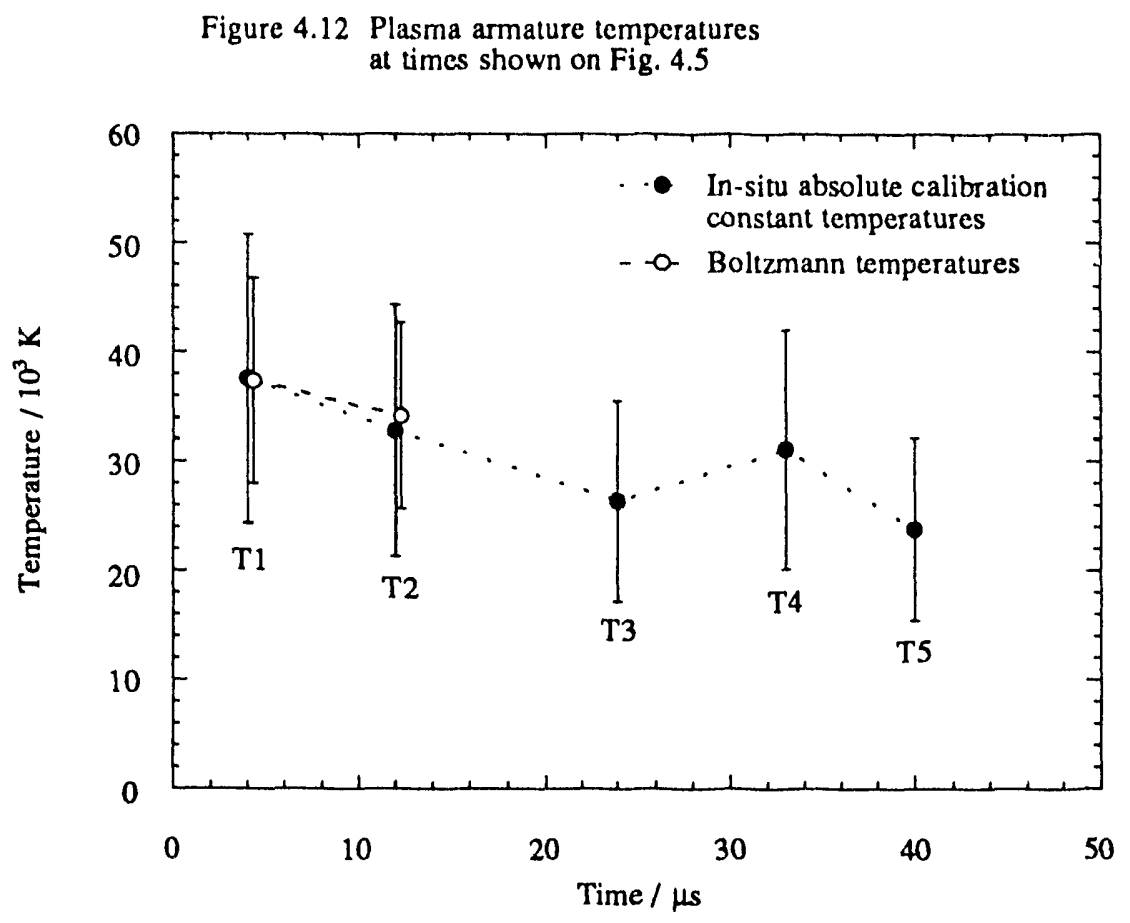

where the uncertainties $\frac{\sigma_{\mathrm{n}_{\mathrm{e}}}}{\mathrm{n}_{\mathrm{e}}}, \frac{\sigma_{\mathrm{A}}}{\mathrm{A}}, \frac{\sigma_{\mathrm{S}}}{\mathrm{S}}$, and $\frac{\sigma_{\mathrm{X}}}{\mathrm{X}}$ were estimated to be $20 \%, 25 \%, 10 \%$, and $20 \%$ respectively. The total estimated uncertainty in the in-situ temperatures was found to be $\pm 35 \%$. These uncertainties are shown as the error bars in Fig. 4.12. Note that because both the electron densities and the Boltzmann temperatures were known at times $\mathrm{T} 1$ and $\mathrm{T} 2$ it was possible to compare the Boltzmann temperatures with those estimated using the in-situ absolute calibration constant. In the Chapter 3 experiments this could not be done because independent values of both the electron density and Boltzmann temperature were known at one time only . 


\section{CHAPTER 5}

\section{TARGET MEASUREMENTS}

\subsection{Introduction}

The final step in this experiment was to determine what effects the plasma armatures would have on targets placed at the railgun muzzle. Chen 5.1 had previously predicted that no emission would be observed from targets impacted with plasmas of the temperatures and velocities attainable with a similar railgun. The measurements performed here provide experimental verification of his predictions.

The spectra emitted during the plasma/target interaction were recorded. The targets were then studied using a scanning electron microscope to determine qualitatively the damage incurred due to the plasma impact. A significant change in the experimental apparatus was introduced for the experiments described in this chapter, namely, the re. "zcement of the photodiode array system with a CCD camera system. The set up and operation of this CCD camera system was the major achievement of the work outlined in this chapter.

\subsection{CCD Camera System Description}

The CCD camera system used in these experiments was purchased from Princeton Instruments, Inc. It is an intensified system capable of being gated to $10 \mathrm{~ns}$ eiectronic shutter widths. The CCD system was purchased with the ultimate intent of being used in a two-photon, laser-induced fluorescence (LIF) experiment aimed at 
determination of the atomic temperature of the railgun-generated plasma-armatures. In addition to allowing for the direct measurement of the atomic temperature of the plasma armatures, an LIF experiment would allow for the plasma temperature to be mapped out across the bore of the railgun, rather than be restricted to line-of-sight integrated temperatures such as those described in Chapters 3 and 4 . This temperature mapping would be another significant improvement in the ability to characterize railgun-generated plasma armatures. An in-depth analysis of such an LIF experiment was conducted and it was determined that a signal-to-noise ratio $\mathrm{S} / \mathrm{N}$ of around 250 could be achieved with experimental conditions similar to those observed thus far (see Appendix $V$ for the details of this analysis). Because of this $\mathrm{S} / \mathrm{N}$ ratio it was felt that an LIF experiment could be conducted, and therefore the camera was purchased.

Although purchased with this LIF experiment in mind, the CCD camera system also offered significant advantages when used for emission spectroscopy compared to the photodiode array (PDA) system used in the previous experiments. First, it is about 100 times more sensitive than the PDA system.5.2 This sensitivity would allow for the detection of much lower emission signals than was possible with the PDA system. Second, it has a minimum gate time of $10 \mathrm{~ns}$, compared to $150 \mathrm{~ns}$ for the PDA system. This higher speed gating would allow for much better resolution of the plasma armatures than was possible with the PDA system. A plasma armature moving at $100 \mathrm{~km} / \mathrm{s}$ could be resolved to $1 \mathrm{~mm}$ long slices using a $10 \mathrm{~ns}$ gate width, compared to a minimum achievable plasma slice of $15 \mathrm{~mm}$ with the PDA system.

The CCD camera system consists of a camera head, a controller/cooler, and a pulser. Contained within the camera head are the CCD device optically coupled to a 
microchannel plate (MCP) image intensifier via an optical-fiber coupler. The optical fiber coupling between the $\mathrm{CCD}$ and the intensifier, together with the high gain of the intensifier and the low readout noise of the CCD array, allow the detector to respond to very low level optical signals. 5.2 The CCD chip is a $576 \times 384$ pixel device composed of $23 \mu \mathrm{m} \times 23 \mu \mathrm{m}$ pixels. Its temperature is maintained at $-35^{\circ} \mathrm{C}$ with a thermoelectric cooler and tap water. Nitrogen is used to flush the photocathode to prevent atmospheric condensation.

The controller is used to regulate the camera head temperature, control its operation, and interface it to a host computer, in this case an IBM PC/AT. Software provided by Princeton Instruments Inc. permitted for relatively simple operation of the system.

The pulser was used to set the width and delay of the pulse sent to the intensifier. It could be operated in either a fixed or variable pulse width mode. The fixed width pulse was specified to have a $10 \mathrm{~ns}$ response time (FWHM). The variable pulse width could be adjusted from 20 to $2000 \mathrm{~ns}$. It was also possible to operate the camera in an open shutter mode in which the optical signal was integrated over the software-specified readout time. This integration time could be increased in $5 \mathrm{~ms}$ increments, beginning at $5 \mathrm{~ms}$. This mode of operation was used when performing optical alignment and calibration of the system. 


\subsection{Alignment and Calibration of the CCD Camera System}

\subsubsection{Mounting of the CCD camera head}

The CCD camera head was coupled to the output of the Spex $0.22 \mathrm{~m}$ spectrometer. This was done by modifying some of the mounting mechanisms used to connect the PDA system to the spectrometer. Tap water and nitrogen were also supplied to the camera head. The mounting scheme allowed the camera face position to be adjusted until it was in the output focal plane of the spectrometer. Because of the two-dimensional nature of the $\mathrm{CCD}$ array, the mounting mechanism was also designed so that the camera head could be rotated. The camera head was mounted to the spectrometer such that there were 576 pixels in the horizontal direction and 384 pixels in the vertical direction. This orientation allowed for the highest degree of spectral resolution. It should be noted here that the orientation chosen resulted in the horizontal pixel numbers varying inversely with the dial setting on the spectrometer. In other words, the spectrum appeared on the monitor starting with the highest wavelength on the left and decreasing with increasing pixel number. The reason this orientation was chosen was for the simple reason that the camera head lettering and controls were right side up using it. Future users of this system would be well advised to invert the camera head so as to avoid the inherent confusion that this orientation caused.

\subsubsection{Alignment}

In order to align the system the output from a helium-neon ( $\mathrm{He}-\mathrm{Ne})$ laser was focussed on the end of the optical fiber used to collect spectral data from the railgun 
shots. The output of the fiber was then focussed on the inlet slit of the spectrometer using the same lenses and mirrors as described in Chapter 2. With the spectrometer dial setting at $632.8 \mathrm{~nm}$ the $\mathrm{He}-\mathrm{Ne}$ line center occurred at pixel number 287, one pixel from the center of the CCD array. The camera head lateral position and its orientation were adjusted until the sharpest output signal was obtained. The focussing optics were then adjusted to improve the signal. These two steps were repeated until no improvement in the sharpness of the optical signal resulted with further adjustment. The camera head position and orientation were then fixed to reduce the chance of subsequent misalignment.

Calibration of the spectrometer/CCD system was also accomplished using a hydrogen lamp. With the 1200 groove/mm grating installed in the spectrometer and with the inlet slit width set at $50 \mu \mathrm{m}$, the $\mathrm{H}_{\beta}$ line center occurred at pixel number 291. For several spectrometer dial settings the pixel number at which the line center occurred was then recorded. In this way the resolution of the system was found to be $0.0572 \mathrm{~nm} / \mathrm{pixel}$. This calibration constant was verified by observing the hydrogen lines at $434.05\left(\mathrm{H}_{\gamma}\right)$ and $410.17\left(\mathrm{H}_{\delta}\right) \mathrm{nm}$. These lines could be observed simultaneously and the distance in pixels between their centers recorded. The center of the spectrometer output varied slightly with the wavelength setting. The $H_{\beta}$ line was centered about pixel 291, while that of the $\mathrm{H}_{\gamma}$ and $\mathrm{H}_{\delta}$ lines occurred at pixels 294 and 296 respectively. This variation was accounted for in the spectral measurements reported here. The resolution of $0.0572 \mathrm{~nm} /$ pixel was slightly better than the $0.0616 \mathrm{~nm} /$ pixel achieved with the PDA system. Using the same procedure, the resolution of the system with the 300 groove/mm grating installed in the spectrometer was found to be $0.225 \mathrm{~nm} / \mathrm{pixel}$. 


\subsection{Experimental Apparatus}

Figure 5.1 shows the experimental setup used with the CCD camera system. The setup was essentially the same as that used in the Chapter 4 experiments. The passive mode of railgun operation was used in these experiments, i.e.the hydrogen was injected between the charged rails. The signal transmitted by an optical fiber.placed upstream of the spectral acquisition fiber was used as a trigger for the CCD camera system in the same way as for the PDA system. It was again necessary to use the waveform generator to set the delay from this trigger until the time of spectral acquisition, because the maximum delay available on the Princeton Instruments pulser was $2 \mu \mathrm{s}$, while delays on the order of $50 \mu \mathrm{s}$ were necessary. The same timing scheme as that used in the Chapter 4 experiments was in these experiments (see Fig. 4.3).

\subsection{Spectral Measurements}

The following approach was used to determine whether any spectral emission from the targets occurred upon plasma impact. First, spectra were acquired in the wavelength region of the $\mathrm{H}_{\beta}$ line to verify that the $\mathrm{CCD}$ camera system was working correctly. Next, the spectrometer was adjusted so that spectra in regions where emission from an aluminum target was likely. The railgun was then fired and the resulting spectra recorded. Aluminum was chosen as the target material because it has relatively few but very strong emission lines. An aluminum foil target was then placed at the muzzle of the railgun, the railgun was fired, and the resulting spectra were recorded with the same experimental conditions as used in the previous step. 
Figure 5.1 Experimental Apparatus

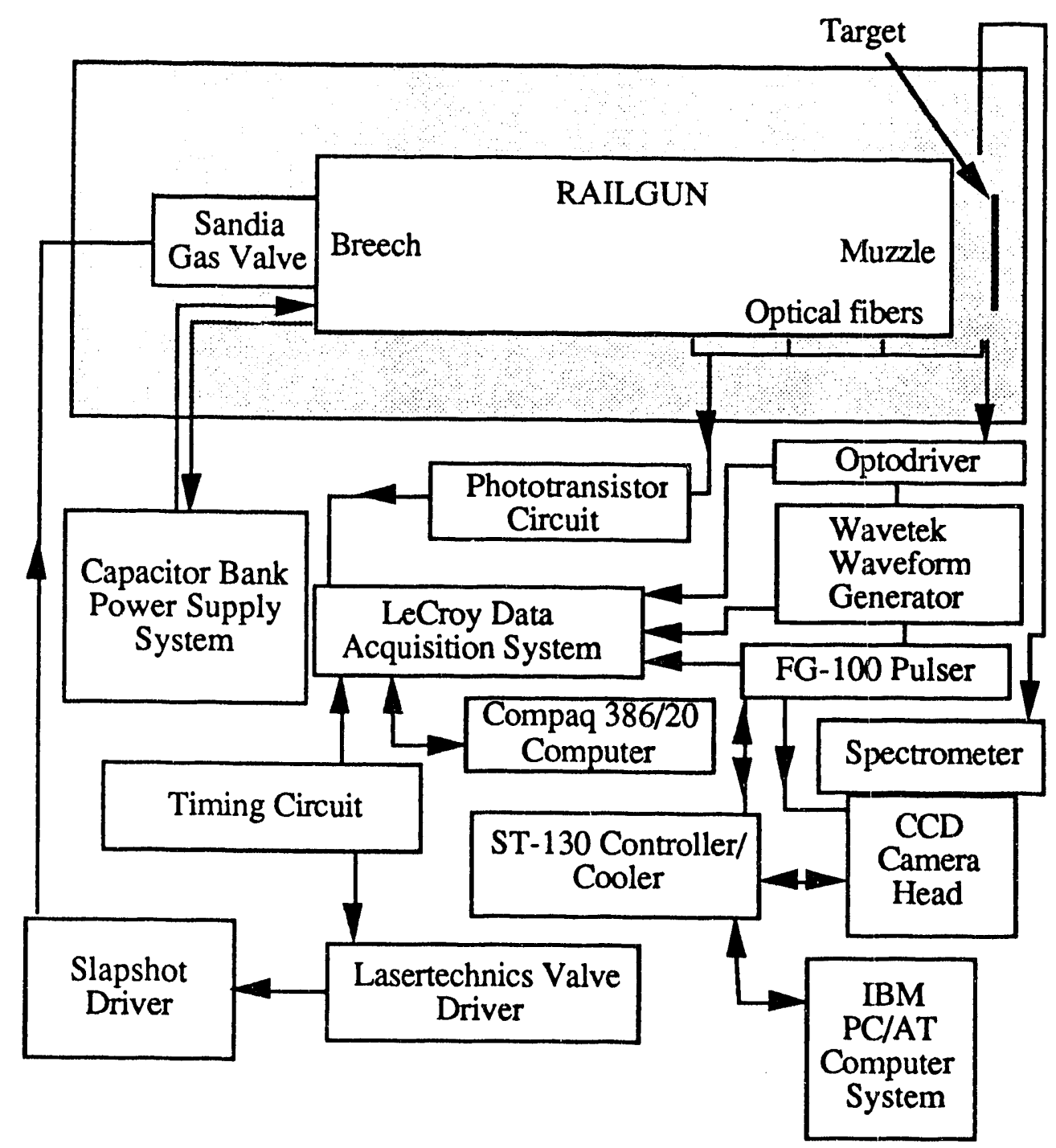

Emission with a stainless steel target was also observed, but because of the large number (thousands) of possible emission lines it was very difficult to determine whether or not target emission occurred. 


\subsubsection{Electron Density Measurements}

To verify that the $\mathrm{CCD}$ camera system was working correctly the spectrometer output was adjusted so that the $\mathrm{H}_{\beta}$ emission line could be observed. It was felt that by making electron density measurements at similar experimental conditions to those used in the Chapter 4 experiments the performance of the CCD camera system could be verified. For the first set of experiments a 300 pixel wide vertical stripe was used to ensure that an adequate optical signal would be obtained. With a $2 \mu$ s gate width, spectra were recorded at 6 and $13 \mu \mathrm{s}$ after arrival of the plasma front (named time TA and TB respectively hereafter). Time TA fell between times $\mathrm{T} 1$ and T2 of Fig. 4.5 and time TB between times T2 and T3.

Figure 5.2 shows the experimental $\mathrm{H}_{\beta}$ profile from time TB along with the nearest theoretical $\mathrm{H}_{\beta}$ profiles. The measured electron density at this point was $4.5 \pm 0.9 \times 10^{22} \mathrm{~m}^{-3}$. If one had drawn a curve through the measured electron densities from the Chapter 4 experiments this electron density would have been very near the predicted value. The electron density measured at TA of $3.7 \times 10^{22} \mathrm{~m}^{-3}$, however, seemed anomalously high. Figure 5.3 shows the electron densities plotted along with those from the Chapter 4 experiments. The reason that the electron density at time TA was so much higher than those at time T1 was that, based on the phototransistor voltage measured at the time of spectral acquisition, the optical signal 
Figure 5.2 Electon density measured $13 \mu \mathrm{s}$ after arrival of the plasma front.

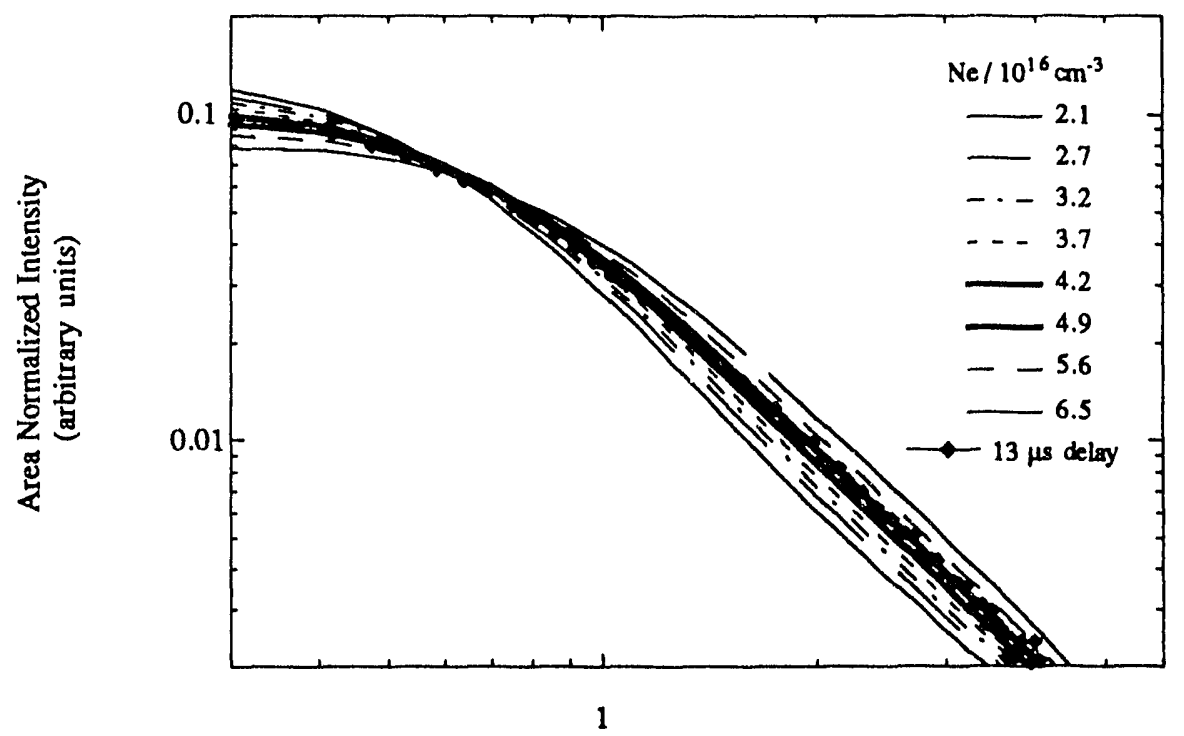

$\Delta \lambda$ from Line Center / nm

Figure 5.3 Electron densities measured using the CCD and PDA camera systems.

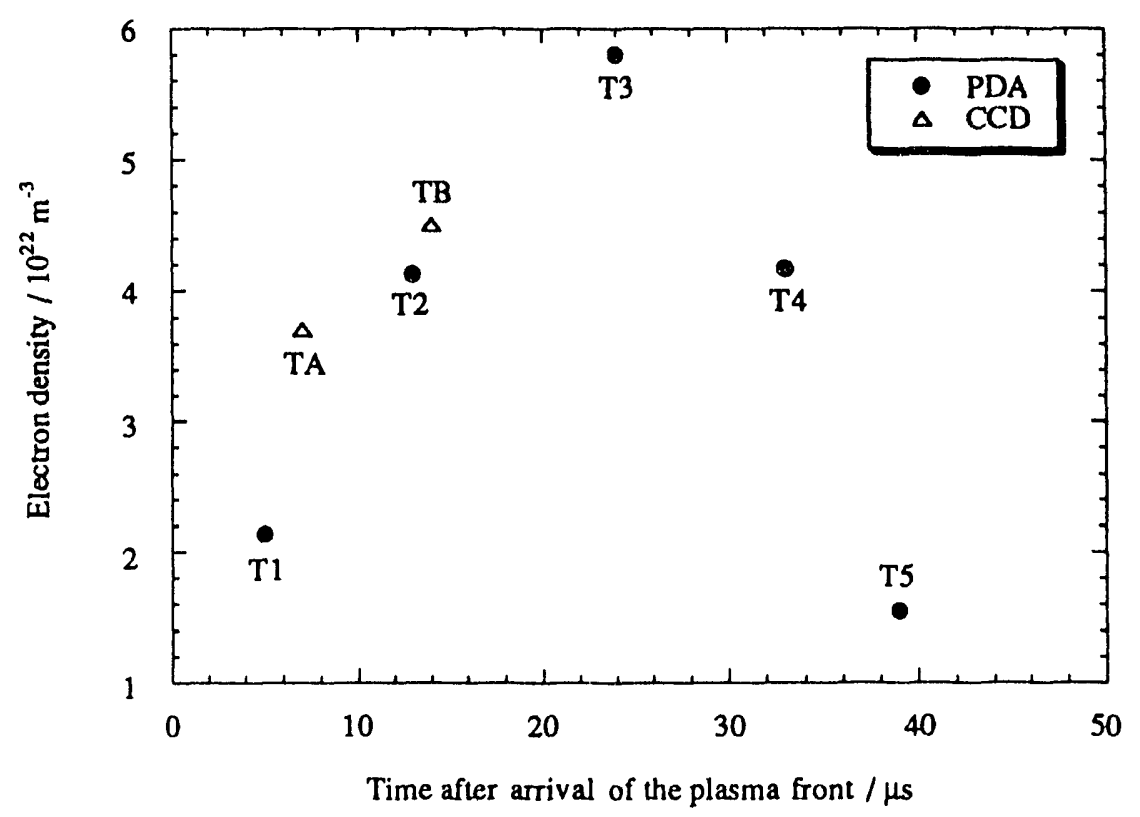


for this shot was much higher than those at T1. For the spectra taken at time $\mathrm{T} 1$ the mean phototransistor signal at F4 of Fig. 4.4 was $0.6 \mathrm{~V}$ while the signal for the same phototransistor was $2.25 \mathrm{~V}$ for spectrum TA. A spectrum obtained at an F4 signal of $2.65 \mathrm{~V}$ in the Chapter 4 experiments had a corresponding electron density of $3.7 \times$ $10^{22} \mathrm{~m}^{-3}$, the same as the value recorded here.

Because of these results it was concluded that the CCD system was mounted and calibrated correctly and that the mode of operation being used to acquire the spectral data was adequate. It was later found that a one pixel wide stripe at the vertical center of the $\mathrm{CCD}$ array could be used to acquire spectra. This mode of operation had the advantage over the use of a 300 pixel wide stripe in that the intensifier gain could then be increased dramatically, thereby taking advantage of the full dynamic range of the CCD pixels. With the 300 pixel wide stripe 4000 to 5000 counts of dark current background accumulated before the spectrum was recorded, compared to about 200 counts for the one pixel wide stripe.

\subsubsection{Target positioning}

A target holder system was constructed which could be positioned using the optical rails attached to the baseplate of the railgun support structure. Figure 5.4 is a schematic diagram of the target holder system. Targets were drawn taught across the opening in the rectangular, lava stone holder before being attached to it with six electrically insulated bolts. The opening in the holder was $25 \mathrm{~mm} \times 75 \mathrm{~mm}$.

The targets used were stainless steel and aluminum foils. These foils were mounted on the target holder such that the spectral acquisition optical fiber could be 
Figure 5.4 Target holder

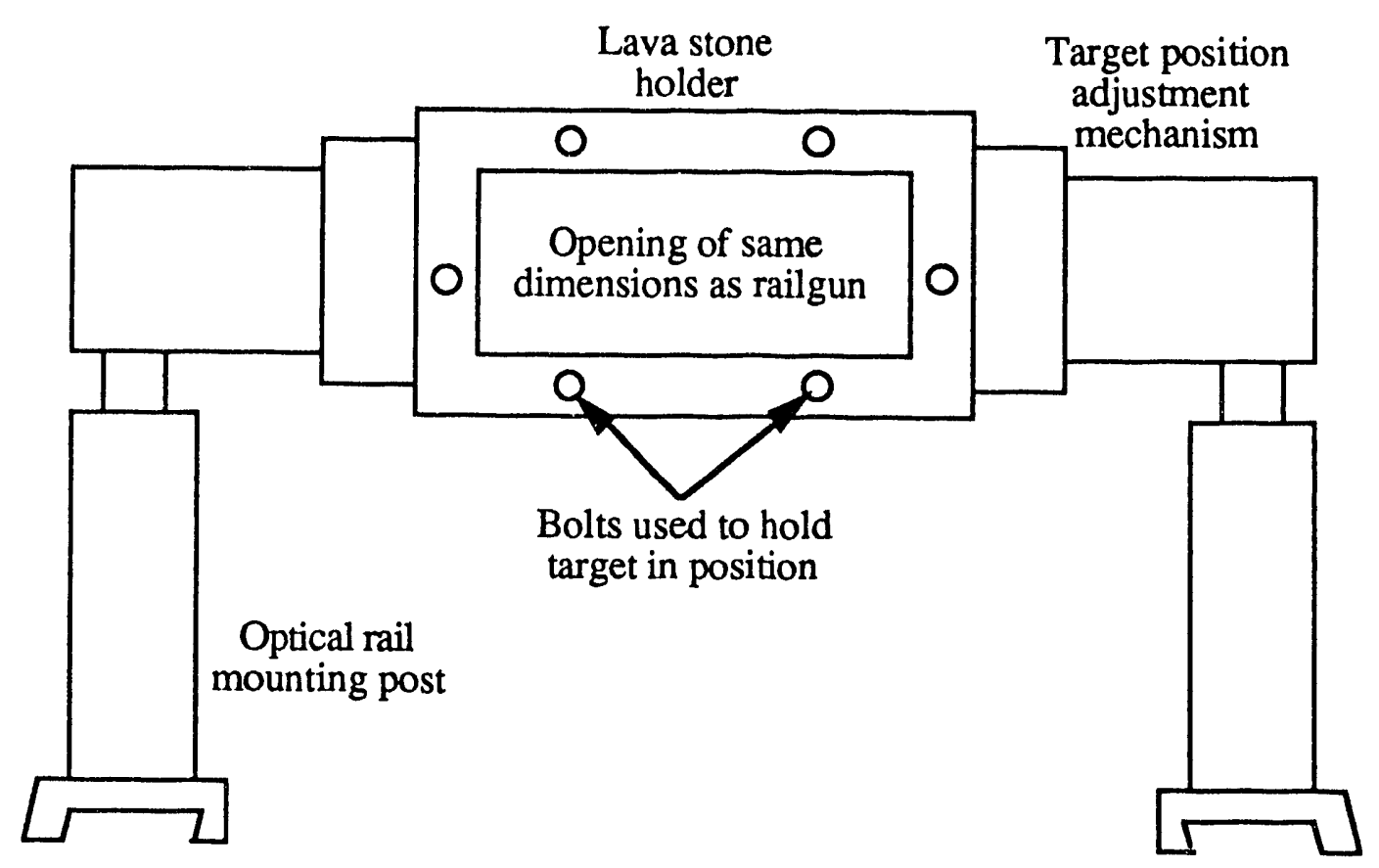

oriented to look along their surfaces, perpendicular to the direction of motion of the plasma armatures. The targets were positioned normal to the railgun bore axis, 175 $\mathrm{mm}$ downstream from the muzzle. The glass insulating sidewalls of the railgun and the Delrin ${ }^{\mathrm{TM}}$ inserts used to electrically isolate the railgun from the support structure were extended out to this position to prevent the plasma from expanding upon exit from the railgun bore. Figure 5.5 is a schematic diagram of the equipment positioning used for these measurements. 
Figure 5.5 Position of Target and Spectral Acquisition Optical Fibers. View from above railgun.

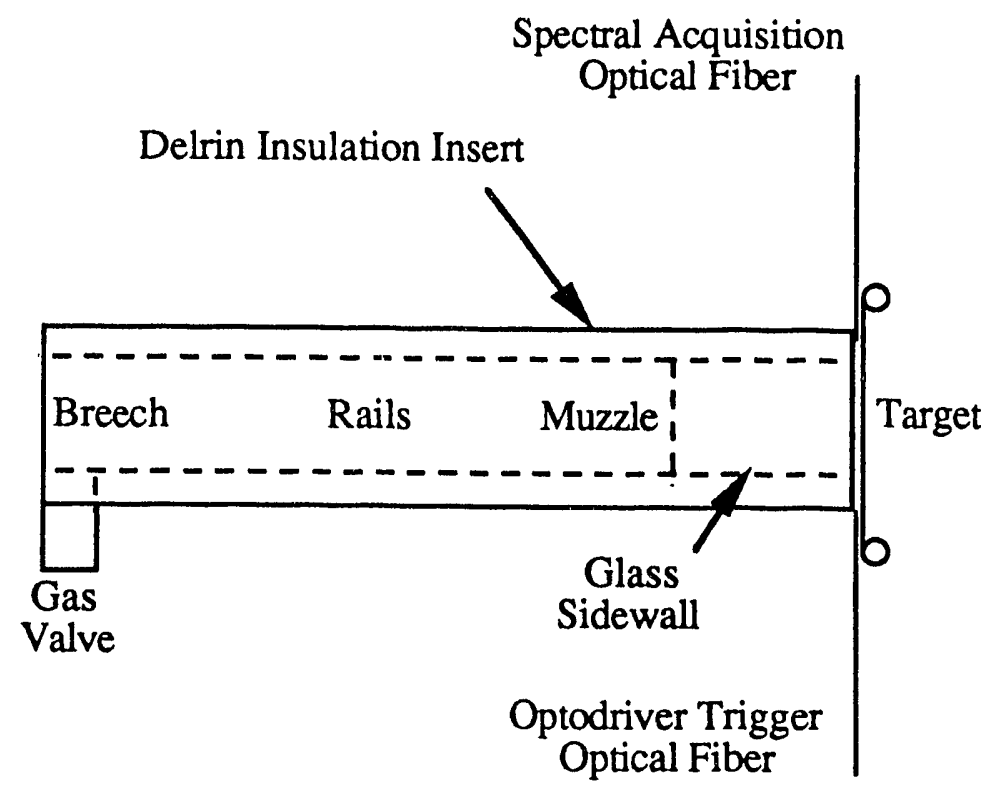

\subsubsection{Target spectral measurements}

The spectrometer was adjusted so that its output would be centered at the strongest emission line of aluminum, $361.5 \mathrm{~nm}$. Figure 5.6 shows the spectrum acquired $17 \mu \mathrm{s}$ after the plasma front reached the target position. Neither the line expected at $361.5 \mathrm{~nm}$, nor another very strong Al I line at $394.4 \mathrm{~nm}$, nor any of the several Al II lines which also occur in this wavelength region were identified. O II lines were present in all of the spectra obtained in the Chapter 3 and 4 experiments 


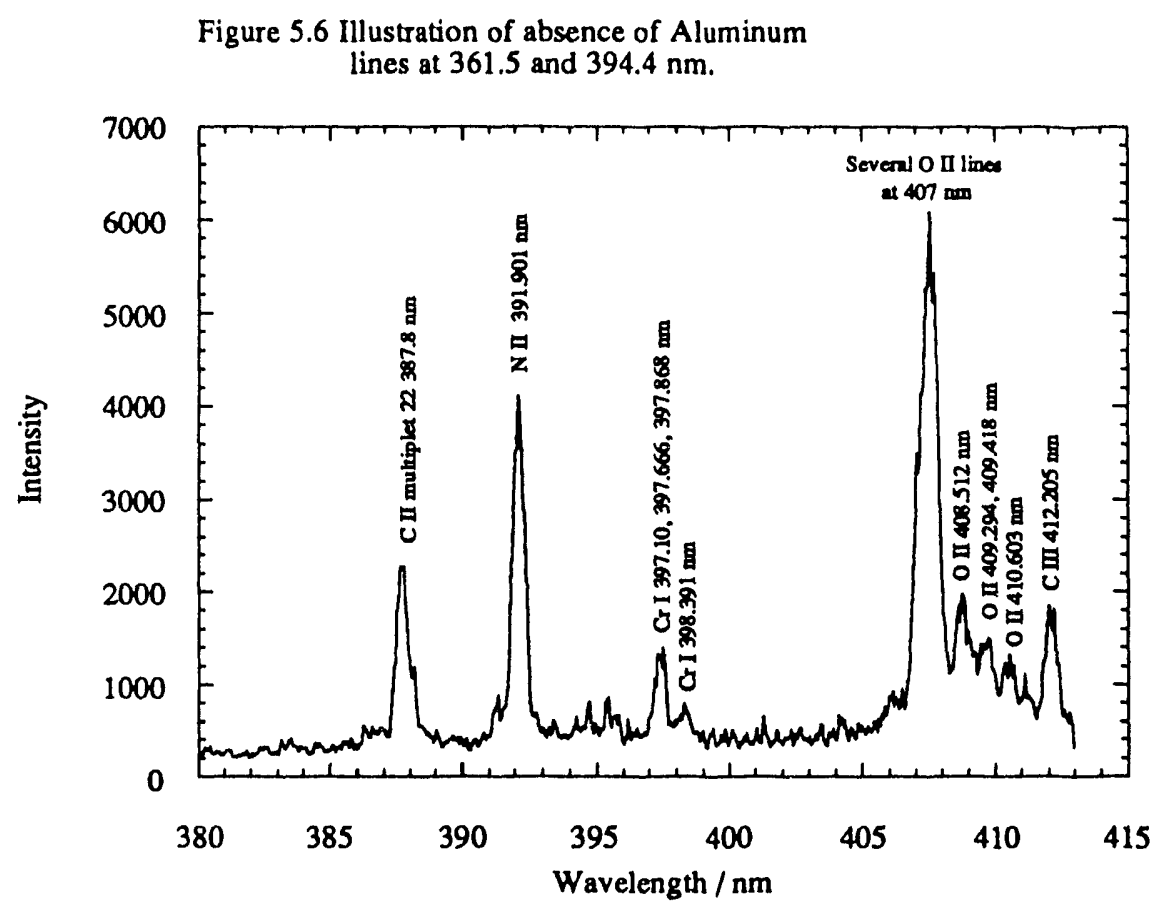

and were expected here. Carbon lines had not been previously identified, but the presence of carbon in the Delrin valve holder and in the electrical isolation inserts shown in Fig. 5.4 make its presence reasonable. Spectra from several other wavelength bands were also observed and in none of these spectra were there clear emission lines from aluminum. These spectra led to the conclusion that this aluminum target was not radiating at the time at which the data were acquired. A more detailed analysis might include the acquisition of spectra at a variety of times after arrival of the plasma armature at the target position, to see if the aluminum radiation is a function of time with which it is bombarded with plasma. 
5.5.4 Target examination with scanning electron microscope (SEM)

An SEM was used to qualitatively examine both the aluminum and stainless steel targets. Targets were observed both before and after being bombarded by the plasma armatures. It was difficult to detect any damage caused by the plasma armatures with the SEM. The surface compositions of the targets were also studied with the SEM. The aluninum targets showed no deposition of any of the plasma elements, while the stainless steel targets did show a small trace of copper after bombardment. It was not particularly surprising that copper was found on the stainless steel targets since the rails of the railgun were made of copper. It did seem strange however that their was no deposition on the aluminum targets. More careful analysis of this phenomenon would be required before any conclusions could be drawn as to why it occurred.

\subsubsection{Target deformation study}

This section outlines the effort that went into the estimation of how much energy was necessary to plastically deform the aluminum foil targets to the extent observed in these experiments.

The aluminum foil targets were stretched taught across the opening in the target holder prior to the railgun shots. After five railgun shots the aluminum foil had plastically bowed to a depth of about $7 \mathrm{~mm}$ from the face of the holder. This deflection represents a strain of about $25 \%$ for the shorter dimension of the opening in the target holder. This is probably the upper bound on the strain, as the strain was considerably less in the longer dimension of the opening and would also be 
corsiderably less if one were to define strain in terms of change in area of the taryet. For arguments sake the mean linear strain will be estimated at $10 \%$.

The strain energy for a material under tension can be expressed as 5.3

$$
U=\int_{L_{0}}^{4} P d x
$$

where $\mathrm{P}$ is the axial force and $\mathrm{x}$ is the direction in which the material is being strained. This integral has both elastic and plastic strain components. For ALCOA ${ }^{\mathrm{TM}}$ alloy 1100 aluminum, the modulus of elasticity $E$ is $70 \mathrm{GPa}$ and the yield strength $\mathrm{Y}$ is $95 \mathrm{MPa}$. The elastic strain estimated as $\mathrm{Y} / \mathrm{E}$ is about $0.1 \%$. Therefore, estimating the total linear strain at $10 \%$ shows that any strain energy calculations would be dominated by plastic deformation.

To quantitatively estimate the strain energy absorbed by the target it would be necessary to do the following two things. First, it would be necessary to impact the target with only a single railgun shot. With several shots fired, the amount of deformation incirred with each shot is not known. Second, stress-strain curves for aluminum foil would be necessary to carry out the integral in Eq. 1. Whether curves such as this exist is unknown. 


\section{CHAPTER 6}

\section{SUMMARY AND CONCLUSIONS}

\subsection{Summary}

The major emphasis of this dissertation was the development of systematic means by which to characterize railgun-generated plasma armatures. In order to do so it was first necessary to design, build, and debug the railgun system. This system included $0.85 \mathrm{~m}$ long, $25.4 \mathrm{~mm} \times 76 \mathrm{~mm}$ rectangular bore railgun. A $1.83 \mathrm{~m}$ long, $0.4 \mathrm{~m}$ diameter vacuum chamber was designed to house the railgun. The vacuum chamber was able to achieve vacuums in the $10^{-6}$ Torr range. An automated data acquisition system capable of acquiring data at up to $5 \mathrm{Msample} / \mathrm{sec}$ was specified, purchased, and successfully operated .

The plasma armatures were formed by the Paschen breakdown of hydrogen gas injected between the charged rails of the railgun. These armatures were then accelerated down the bore of the railgun by the resulting $\mathbf{J} \times \mathbf{B}$ force. Several sets of diagnostic devices were designed and built with which the armatures were characterized. A Rogowski coil was used to measure the railgun current as a function of time, and a maximum current of $25 \mathrm{kA}$ was reccrded $30 \mu \mathrm{s}$ after initiation of the railgun discharge. Phototransistors and $\stackrel{\circ}{\mathrm{B}-p r o b e s}$ were used to measure the velocities of the plasma armatures. Using these devices a maximum plasma front velocity of $150 \mathrm{~km} / \mathrm{s}$ was recorded at a distance of $0.60 \mathrm{~m}$ from the railgun breech. The velocity slowed to about $10 \mathrm{~km} / \mathrm{s}$ at a distance of $0.98 \mathrm{~m}$ from the breech. 
A gated, intensified, photodiode-array, spectral-acquisition system was used to measure the spectra emitted at several points within the plasma armatures. Near the front of the armatures the plasma contained oxygen, nitrogen, silicon, and carbon, in addition to hydrogen. The impurities in the armatures quickly decreased with increasing distance from the armature front. Electron density profiles of the plasma armatures were determined by matching the measured profiles of the $\mathrm{H}_{\beta}$ emission line with profiles arrived at by convolving theoretical $\mathrm{H}_{\beta}$ profiles with the instrumental-broadened $\mathrm{H}_{\beta}$ line obtained from a hydrogen lamp. The maximum measured electron density was $5.8 \times 10^{22} \mathrm{~m}^{-3}$. Electron temperature profiles of the plasma armatures were calculated using the intensities of O II emission lines. The maximum electron temperature of the armatures was found to be $4.1 \times 10^{4} \mathrm{~K}$.

A new method was devised by which the absolute calibration constant of the spectral acquisition system could be determined based on measured electron densities and temperatures. Once determined, this calibration constant was used to estimate the electron density of the plasma at points where the temperature was known, or conversely, to estimate the temperatures at points where the electron densities were known.

Finally, a CCD-based, spectral acquisition system was specified, purchased, and successfully operated. This system is capable of recording very low level optical emission at very high speeds (electronic shutter widths down to $10 \mathrm{~ns}$ ). Spectral measurements similar to those described above were conducted with this camera to verify its operation. It was then used to determine that the plasma reflected by an aluminum target contained no aluminum. 
The methods of plasma armature characterization demonstrated in this dissertation should be applicable to other railguns with similar operating characteristics. The temperature, electron density, and composition measurements of the railgun-generated plasma armatures are the most detailed and systematic measurements of their kind. Other researchers should find application of these methods useful in determining the operating characteristics of their railguns.

\subsection{Concluding Remarks}

This dissertation was devoted to the development of an plasma-armature railgun facility. Methods were detailed by which key parameters of plasma armatures could be measured. The usefulness of this facility is, however, far from exhausted. Many other experiments could be performed with the existing equipment, and with the purchase of only a couple more pieces of instrumentation still more interesting measurements of the plasma armature characteristics could be performed.

The existing equipment could be used study other characteristics of the plasma armatures. For instance, because of its ability to acquire two-dimensional optical images, the CCD camera could be used to measure the distribution of the plasma within the bore of the railgun. If one were trying to obtain a uniform plasma distribution for use in a material deposition study, this measurement would be vital. Because of the short gate width available on the CCD camera system it might also be possible to measure atomic properties of the plasma armatures such as transition probabilities. And the use of gases other than hydrogen might allow for the study of material deposition by plasma armatures on substrates of interest to the Film coating industry. Finally, with the existing hardware, parametric studies of how the plasma 
characteristics vary with changing voltages, valve pressures, chamber pressures, etc. could be studied.

There exists hardware which, when coupled to the CCD camera, would allow for simultaneous, multi-point, spectral acquisition. Specifically, a spectrometer which maintains its vertical resolution would be required along with the correct focussing optics. Princeton Instruments Inc. engineers claim to have resolved 18 spectra simultaneously on a single camera such as the one used here. The ability to measure multiple points simultaneously would allow for instance, for the entire temperature profile of a plasma armature to be measured on a single shot. This would be a significant improvement in the capability of the spectral acquisition system.

Finally, with the acquisition of the correct laser system, it would probably be possible to conduct fluorescence measurements at specific points within the plasma armatures. Appendix $\mathrm{V}$ is a feasibility analysis of such a two-photon LIF measurement. Based on the knowledge gained from the completion of this study, this LIF measurement would probably be sufficiently complex for another doctoral student to undertake it as his dissertation subject. 


\title{
APPENDIX I \\ EQUIPMENT SPECIFICATIONS
}

\begin{abstract}
A1.1 Vacuum pumping system
The roughing pump is a Welch Duo Seal Vacuum Pump (model 1374), made by Sargent-Welch Scientific Co. It is driven by a 1 HP General Electric motor. The cryogenic (cryo) pump is a Varian VK-12C with a helium-cooled compressor module.
\end{abstract}

Chamber pressure was monitored via two thermocouple gauges (Vacuum Gauge and Test Thermocouple Vacuum Gauges) and one ionization gauge (Granville-Phillips model \# 274002). One thermocouple gauge and the ionization gauge were situated on the cryo-pump side of the main gate valve (see Fig. 2.1), and the other thermocouple gauge was located on the main chamber side of the main gate valve. This scheme allowed for the cryo-pump pressure to be monitored independently of the main chamber pressure. Both the thermocouple and the ionization gauge outputs were monitored with a Granville-Phillips Series 270 Ionization Gauge Controller, mounted as shown in Fig. 2.1.

\section{A 1.2 Optical rails}

Newport Micro-Optical rails and mounts were used for positioning of the diagnostic equipment along the side of the railgun. These rails and mounts were 
chosen because of their small size and because they are made of anodized aluminum, which is a good vacuum material.

\section{A1.3 Hydrogen pressure gauge}

The pressure gauge shown in Fig. 2.4 is an Edwards Datametrics Barocel Pressure Sensor, Type 600A-1000,JT-R12-h22L-X, Range 10000 Torr. It was controlled using an Edwards Datarnetrics 1500 Digital Pressure Display and Power Supply.

\section{A1.4 Piezoelectric hydrogen valve}

The piezoelectrically-actuated valve used to inject hydrogen into the railgun bore was a Lasertechnics Model LPV Molecular Beam Valve. A driver for this valve was also purchased from Lasertechnics (LPV Valve Driver). We were never able to overcome the noise problems which caused the valve to remain open after actuation, so this valve was not used in any of the final experiments.

\section{A1.5 Sandia valve driver}

The power supply used to drive the solenoid valve obtained from Sandia National Laboratories was a Plasma Research Corporation Slapshot Pulser, Model $1 \mathrm{KS}$. The output voltage of this supply could be continuously adjusted from $100 \mathrm{~V}$ to $1200 \mathrm{~V}$. 


\section{A1.6 Capacitor bank}

The capacitor bank used to power the railgun contained three $170 \mu \mathrm{F}$ nominal capacitors. Two of these were $10 \mathrm{kV}$ Maxwell Laboratories Energy Discharge Capacitors, Cat. \# 30013, with measured capacitances of 177 and $173 \mu \mathrm{F}$. The other was a $10 \mathrm{kV}$ Sangamo Energy Discharge Capacitor, with a measured capacitance of $167 \mu \mathrm{F}$.

\section{A1.6 Ignitron}

The ignitron used to initiate discharge of the capacitor bank was a General Electrics \#GL-37207A.

\section{A1.7 Capacitor bank power supply}

The capacitor bank was charged using a Universal Voltronics Corporation High Voltage DC power supply (Model No. BAS 22-160, 22 kV, $160 \mathrm{~mA}$ ).

A1.8 Rogowski coil and current viewing resistor (CVR)

The Rogowski coil was constructed by wrapping magnetic wire around a standard BNC coaxial cable, $4.8 \mathrm{~mm}$ outer diameter. It was $300 \mathrm{~mm}$ long with 1 turn $/ 25 \mathrm{~mm}$. The CVR used to calibrate it was a T\&M Research Products Inc. K10000-20 Current Viewing Resistor. The measured resistance of this CVR was $0.4865 \mathrm{~m} \Omega$. 


\section{A1.9 Fast ionization gauge}

The ionization gauge used in the hydrogen propagation experiment was a Beam Dynamics, Inc. Model FIG-1 It has a $3 \mu$ s response time, a useful DC pressure range of $10^{-8}$ to $10^{-2}$ Torr, and a sensitivity of $1 \times 10^{5}$ Volt Torr $^{-1} \mathrm{~A}^{-1}$. Included in this purchase was the Model FC-1 Control Unit and the Model FH-1 Gauge Head.

\section{A1.10 Data acquisition system}

The computer used to drive the data acquisition system was a Compaq $386 / 20$ personal computer. A IEEE 488 card was installed in the computer so that it could communicate with the CAMAC crate. A VGA color graphics card was also installed in the computer so it could run the software which drives the waveform recorders.

Two LeCroy 6810 Waveform Recorders (6810's) was used for data acquisition. The 6810 module is a standard CAMAC module and a LeCroy GPIB module was used to interface the CAMAC with the computer.

\section{A1.11 Spectral acquisition system}

A Spex $1681 \mathrm{C}, 0.22 \mathrm{~m}$ spectrometer was used for spectral acquisition. This spectrometer could be fitted with either a 1200 groove/mm grating (Bausch \& Lomb 35-33-10-200) for narrow band, higher resolution spectral acquisition or a 300 groove/mm grating (Spex 1506 - 500A) for wide band, lower resolution spectral acquisition. This spectrometer was fitted with a Spex $1697 \mathrm{~F}$ inlet slit which is continuously adjustable from $25 \mu \mathrm{m}$ to $8 \mathrm{~mm}$. The He-Ne laser used for alignment of the spectral acquisition systems was built by Hughes Aircraft Company and had a 10 
$\mathrm{mW}$ output. The laser output was focussed on the fiber optic cable used for spectral acquisition inside the vacuum chamber. The fiber output was focussed on the inlet slit to the spectrometer, via a pair of achromatic lenses (Spindler and Hoyer, Inc., 30 $\mathrm{mm} / 11.5$ and $100 \mathrm{~mm} / 24)$.

\section{A1.12 Photodiode array system}

The photodiode array system used in the Chapter 3 and 4 experiments was a Tracor Northern, 512 element, intensified, gatable, linear, photodiode array system. It is described in detail in Ref. 2.3.

\section{A1.13 CCD camera system}

The CCD camera system used in Chapter 5 was a Princeton Instruments Inc, (P.I.), intensified, gatable, 2-dimensional, CCD camera system. The individual components of this system are listed below along with their description, specifications, and capabilities.

i) P.I. FG-100 Pulser and Intensifier

1) The gate width of the intensifier was continuously variable from $20 \mathrm{~ns}$ to $2500 \mathrm{~ns}$ or it could be set at a fixed width $10 \mathrm{~ns}$ (gate width was defined as the full width at hali maximum of the peak opening of the tube at its maximum gain). The delay was continuously adjustable from 20 ns to $1700 \mathrm{~ns}$, with fixed delay of $11 \mathrm{~ns}$ also available.

2) The intensifier had a quartz input window which allowed wide spectral acquisition (see 3 below).

3) It had an $18 \mathrm{~mm}$ diameter, red-blue-enhanced photocathode with the following spectral response (SR) characteristics:. 


$\begin{array}{cc}\begin{array}{c}\text { Wavelength } \\ \mathrm{nm}\end{array} & \begin{array}{c}\text { Response* } \\ \mathrm{mA} / \mathrm{W}\end{array} \\ 200 & 16 \\ 250 & 19 \\ 300 & 23 \\ 350 & 31 \\ 400 & 37 \\ 450 & 38 \\ 500 & 42 \\ 550 & 39 \\ 600 & 36 \\ 700 & 28 \\ 800 & 16 \\ 900 & 1\end{array}$

*The spectral response at each wavelength is guaranteed to be greater than or equal to the listed value.

5) Single microchannel plate.

6) P-20 phosphor screen.

7) Fiber optic output.

8) Typical luminous gain of $12,000 \mathrm{ft}$-lambert/ft-candle. 
ii) P.I. ICCD-576 (intensified CCD) Camera head

1) Fiber optic window input. The system was delivered with the fiber optic output of the intensifier coupled to the fiber optic input window of the CCD camera head. The CCD camera and intensifier were packaged as a single unit which was mounted directly onto the output of the spectrometer.

2) $384 \times 576,23 \mu \mathrm{m} \times 23 \mu \mathrm{m}$ pixels.

3) Geometric distortion of less than one pixel.

4) Dynamic range of 14 bits.

5) Response linear to within $1 \%$ for upper $95 \%$ of range.

6) Operating temperature $-35^{\circ} \mathrm{C}$ with tap water circulation. A Nitrogen purge was also required to prevent condensation on the faceplate of the intensifier.

7) Dark current less than or equal to 12 electrons/pixel/s.

8) Read out noise less than 25 electrons/pixel 100 kpixels/s.

iii) P.I. ST-130 Controller for camera head and intensifier.

1) Was used to cool and control the operation of the camera head/intensifier system.

2) Allowed the user to define pixel stripes (1 to 384 stripes) and bin size (pixels to be binned together ( 1 to 384 ) for each individual stripe). This binning capability allowed for great flexibility in how data was acquired (see Chapter 5). 
3) Skip mode which allowed skipping of irrelevant pixels along the 576 pixel row direction. This feature greatly enhanced the rate at which data could be analyzed.

4) Allowed for pixel summation along the 576 pixel row direction

iv) P.I. ICCD driver software, version 1.31.

1) This IBM-compatible software allowed for complete camera system and data acquisition setup. Several system configurations were provided by P.I. for driving the camera. One of these, named R1, was used for acquisition of the data discussed in Chapter 5.

2) Provided psuedo-real-time display of images from multiple, softwaredefined superpixels (bins).

3) Included vertical and horizontal zooming during image display.

4) Provided mathematical functions for frame manipulation, including addition and subtraction.

5) Graphics and plotting capability.

v) Computer

1) The controller was driven using an IBM PC AT computer. The PC memory was expanded to 6 Mbytes to avoid any compatibility problems with the controller. The Compaq 386/20 described above was originally intended to drive the controller, but was used to drive the LeCroy system instead. A minor drawback to this arrangement was the reduction in speed of the system. The IBM PC/AT is a 80286-based machine with an 80287 coprocessor. 
vi) Data Translation DT2861 Frame Grabbber Board

1) The primary use of this computer board is for image acquisition and manipulation. It was not necessary to use this board for spectral acquisition.

vii) Monitor

1) A Mitsubishi FA3425K Series Multisync color monitor was used for high-resolution display of the data.

A1.14 Wavelength calibration lamps and power supply

The iodine and hydrogen lamps used for wavelength calibration of the PDA system were built by Central Scientific Company (CENCO) and were powered by a $30 \mathrm{~mA}$ Transco Inc. Transformer (Catalog \# T7512).

\section{A1.15 Tungsten filament lamp and power supply}

The tungsten filament lamp used to obtain the instrumental response functions of the spectral acquisition systems was a General Electric 18A/T10/1P-6V, -8 filament, Med. Pref. base down, ASA code-EDT. This lamp was powered by a $1 \%$ stabilized power source (Sola Electric, 5.2 V DC, 15 A, cat \# 28-05-315).

\section{A1.16 Optical pyrometer}

The optical pyrometer used to determine the temperature of the tungsten filament lamp was made by Leeds \& Northrup Co. (Cat No. 8622). 


\section{APPENDIX II \\ DETAILS OF ELECTRON DENSITY CALCULATIONS \\ BY THE GRAPHICAL METHOD}

\section{A2.1 Introduction}

This appendix details the method by which the theoretical $\mathrm{H}_{\beta}$ profiles of Vidal et al. 3.6 were modified to allow for comparison with the experimentally-obtained $\mathrm{H}_{\beta}$ profiles. The theoretical $\mathrm{H}_{\beta}$ profiles account for Stark-broadening and Doppler broadening only. However, the instrumental broadening of our spectrographic system was measured and its FWHM was found to be about $4 \AA$. To account for this added broadening it was necessary to convolve the theoretical profiles with the instrumental profile before comparing them with our experimentally measured profiles. The Pascal computer programs used in these calculations were written using Borland's Turbo Pascal ${ }^{\top M}$ and are included at the end of this appendix in the order in which they are discussed.

\section{A2.2 Data Conversion}

The $\mathrm{H}_{\beta}$ lineshape data listed in Ref. 3.6 is listed at electron densities chosen in such a way that when they are plotted on a log-log scale they are equally spaced. They have been normalized such that if $I$ is the intensity of a line at a given wavelength $\Delta \lambda$ from the center of the $\mathrm{H}_{\beta}$ line $(\lambda=4861.3 \AA)$, then the normalized intensity $S$ and wavelength $\Delta \alpha$ are given by 


$$
\Delta \alpha=\Delta \lambda / \text { Fo, }
$$

and

$$
\mathrm{S}=\mathrm{I} \times \mathrm{Fo}
$$

where

$$
\mathrm{Fo}=1.29 \times 10^{-9} \mathrm{ne}^{-2 / 3}
$$

The first step necessary then was to convert this normalized S vs. $\Delta \alpha$ data into I vs. $\Delta \lambda$ form (see Program Conversion). The experimental data could not be normalized using Fo because the electron densities of the experimental data were not known a priori. The theoretical profiles were provided at electron densities chosen such that they were evenly spaced on a $\log -\log$ plot,. g.,. at $1 \times 10^{15}, 3.162 \times 10^{15}$, and $1 \times$ $10^{16} \mathrm{~cm}^{-3}$. At each electron density profiles were provided for temperatures of 2500 , $500,10000,20000$, and $40000 \mathrm{~K}$. Because plasma temperatures near $20,000 \mathrm{~K}$ were estimated using the Boltzmann plots of Chapter 3, the profiles at 20,000 K were used in this work. Also, the electron densities of the plasma armatures was roughly estimated to be in the $10^{16} \mathrm{~cm}^{-3}$ range, based on some crude full width at half maximum measurements of the experimental $\mathrm{H}_{\beta}$ profiles. Therefore, intermediate profiles were generated in the $10^{15}$ to $10^{17} \mathrm{~cm}^{-3}$ range.

\section{A2.3 Interpolation}

The next step was to calculate the theoretical intensities at wavelengths with which it would be possible to convolve the experimentally-obtained instrumental broadening function. The theoretical profiles were provided such that the data points would be evenly spaced on a log-log plot. It was necessary to interpolate points which were evenly spaced in linear space for convolution with the instrumental function. The instrumental function was measured using the photodiode array system 
and a hydrogen lamp, and was known at a spacing of $0.616 \AA$, the resolution of the photodiode array system. The program written to do this was adapted from Bevington. 3.8 See program Program Interp_Theoretical_Intensity. It employs a polynomial interpolation scheme (Eqs.13-11 and 13-12 of Ref. 3.8) in which four data points surrounding the desired point are used.

\section{A2.4 Intermediate curve generation}

Next, the same interpolation scheme was used to generate intermediate $\mathrm{H}_{\beta}$ profiles between those of Vidal et al. The profiles generated in section A2.3 at electron densities of $1 \times 10^{15}, 3.162 \times 10^{15}, 1 \times 10^{16}, 3.162 \times 10^{16}$, and $1 \times 10^{17}$ $\mathrm{cm}^{-3}$ and $20,000 \mathrm{~K}$ were used to generate the profiles at intermediate electron densities (see Program H_beta_curve_Interp). We also chose to generate these intermediate profiles at electron densities evenly spaced on log-log scale because plotting the data on a log-log scale allows for better comparison of the wings of the theoretical data with those of the experimental data. This is important because the theoretical profiles have been found to best match those obtained experimentally.in the wings. 3.6

\section{A2.5 Instrumental profile generation}

Before convolution of the theoretical profiles with the measured instrumental broadening function could be performed it was necessary to generate instrumental intensities at the same wavelengths as those of the theoretical profiles. In addition, it was necessary to subtract the background from the measured profile. See Program H_Beta_Profile for a listing of this routine. These profiles were also normalized by area. 


\section{A2.6 Convolution}

With the theoretical and instrumentally broadened profiles now known at wavelengths of $\Delta \lambda=0,1,2,3, \ldots .50 \AA$ from the center of the $\mathrm{H}_{\beta}$ line it was now possible to convolve the two. The accuracy of the convolution routine was verified by convolving two Lorentzian curves. The convolution of two Lorentzian curves is also Lorentzian, with a FWHM equal to the sum of the FWHM's of the convolved curves. The convolution routine was found to produce this result (see Program Convolution). Each theoretical curve generated above was convolved with the measured, instrumental broadening function measured using an liydrogen lamp. The convolved profiles were area normalized. Area normalization was found to produce the best match between the railgun-generated $\mathrm{H}_{\beta}$ profiles and the convolved profiles.

\section{A2.7 Railgun shot data manipulation}

The final step in the process was to format the railgun spectral data for comparison with the above profiles. This formatting included area normalization of the data as well as converting the abscissa from pixels to Angstroms (see Program Wing_create). The profiles were now ready for graphical comparison with the theoretical curves. The software package Kaleidagraph ${ }^{T M}$ was found to be easy to use for this purpose. 
Program Conversion;

\{ This program converts the line strengths and dimensionless wavelengths in (Vidal,Cooper,and Smith) to normalized intensities and wavelengths in angstroms for comparison with the experimental data.)

\{\$U Graph2D)

uses Memtypes, Quickdraw, Graph2D;

type

wave $=$ array $[1 . .50]$ of real;

var

$\mathrm{i}, \mathrm{j}, \mathrm{k}, \mathrm{kk}$ :integer;

a,b,c,d:text;

ne,Fo,dd:double;

s,Alpha,int,l:wave;

$\{* *+1$

Procedure Normalize (k:integer, var s:wave);

\{This procedure normalizes the array s of length $\mathrm{k}$ to unity\}

var

$\mathrm{i}, \mathrm{j}:$ integer,

max:double;

begin

$\max :=\mathrm{s}[1]$;

for $\mathrm{i}:=2$ to $\mathrm{k}$ do

if $s[i]>\max$ then $\max :=s[i]$;

for $i:=1$ to $k$ do

$\mathrm{s}[\mathrm{i}]:=\mathrm{s}[\mathrm{i}] / \max$;

end; \{of procedure normalize \}

$\{* * * * * * * * * * * * * * * * * * * * * * * * * * * * * * * * * * * * * * * * * * * * * * * * * * * * * * * * * * * * * * * *\}$

Procedure Match (k:integer; yy:wave; var xx:ar);

(Used to convert array lengths for use in procedure Splot. All arrays plotted using Graph2D must have arrays of type "ar"

Begin

for $\mathrm{i}:=1$ to $\mathrm{k}$ do $\operatorname{xx}[\mathrm{i}]:=y y[i]$; 
end; \{Procedure Match\}

$\{* * * * * * * * * * * * * * * * * * * * * * * * * * * * * * * * * * * * * * * * * * * * * * * * * * * * * * * * * * * * * * * *\}$

Procedure Splot (k:integer, r,laml:wave);

(Plot using graghics package Graph2D of Borland's Turbo Pascal ${ }^{\mathrm{TM}}$. "Drawline" is a command which sends an $\mathrm{X}-\mathrm{Y}$ variable set to the graphics package for plotting.)

var

i,n:integer,

$\mathrm{xmax}, \mathrm{xmin}, \mathrm{ymax}, \mathrm{ymin}: \mathrm{real}$;

$x x, y y: a r$;

Begin

clearscreen;

$\mathrm{n}:=\mathrm{k}$;

-anlax: $=0$;

$x \min :=0$;

$y \max :=0$;

ymin: $=0$;

Match(k,laml,xx);

$\operatorname{Match}(k, r, y y)$;

drawline 1 (xx,yy,n,xmax,xmin,ymax,ymin);

readlin;

End; \{Procedure Splot\}

$\{* * * * * * * * * * * * * * * * * * * * * * * * * * * * * * * * * * * * * * * * * * * * * * * * * * * * * * * * * * * * * * * *\}$

Begin \{Main\}

$\mathrm{kk}:=25$;

reset(a,'alpha.dat');

for $\mathrm{i}:=1$ to $\mathrm{kk}$ do

readln(a, alpha[i]);

close(a);

reset(a,'ss 1.dat');

Rewrite(b,'i1.dat');

Rewrite(c,'11.dat');

readln(a,k);

readln(a,ne); 


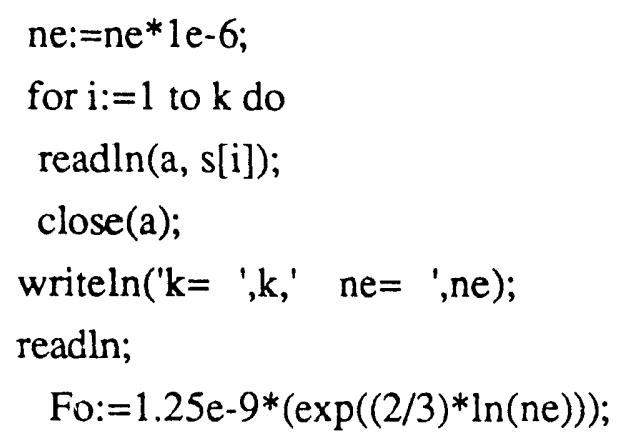

\{Convert $S$ and alpha to I and lambda respectively\}

For $\mathrm{i}:=1$ to $\mathrm{k}$ do

Begin

1[i]:=alpha[i]*Fo;

int $[\mathrm{i}]:=\mathrm{s}[\mathrm{i}] / \mathrm{Fo}$;

End;

dd:=int[1];

for $\mathrm{i}:=1$ to $\mathrm{k}$ do

$\operatorname{int}[\mathrm{i}]:=\operatorname{int}[\mathrm{i}] / \mathrm{dd}$;

for $i:=1$ to $k$ do

begin

writeln(b,int[i]:16:8);

writeln(c,1[i]:16:8);

end;

Close(b);

close (c);

$\mathrm{k}:=15$;

Splot(k,int,1);

End. 
Program Interp_Theoretical_Intensity;

\{This program calculates the theoretical intensities at wavelengths chosen for comparison with the experimental values, namely, at delta lambda $=1,2,3 \ldots 50$ angstroms from the center of the $\mathrm{H}$ beta line.)

\{\$U Graph2D)

$\{\$ I-\}$

uses Memtypes, Quickdraw, Graph2D;

type

wave $=$ array $[1 . .75]$ of real;

$\mathrm{f}=$ file of real;

var

$\mathrm{i}, \mathrm{j}, \mathrm{k}, \mathrm{kk}, \mathrm{ii}, \mathrm{t}$ :integer;

a,b,c,d:text;

int, w,intc, ww, $x, y:$ wave;

p:array [1..75] of integer;

$\mathrm{xx}, \mathrm{yy}:$ real;

ss,tt:ar;

$\{* * * * * * * * * * * * * * * * * * * * * * * * * * * * * * * * * * * * * * * * * * * * * * * * * * * * * * * * * * * * * * * *\}$

Procedure Match (k:integer; qq:wave; var $\mathrm{xx}: \mathrm{ar}$ );

Begin

for $\mathrm{i}:=1$ to $\mathrm{k}$ do

$x x[i]:=q q[i] ;$

end; \{Procedure Match\}

$\{* * * * * * * * * * * * * * * * * * * * * * * * * * * * * * * * * * * * * * * * * * * * * * * * * * * * * * * * * * * * * * * *\}$

Procedure Splot (k:integer; w,int:wave; kk:integer, ww,intc:wave);

\{Plot using graghics package Graph2D\}

var

i,n:integer;

$x \max , x \min , y \max , y \min$ :real;

$x x, y y: a r$;

Begin

clearscreen;

$\mathrm{xmax}:=0$; 


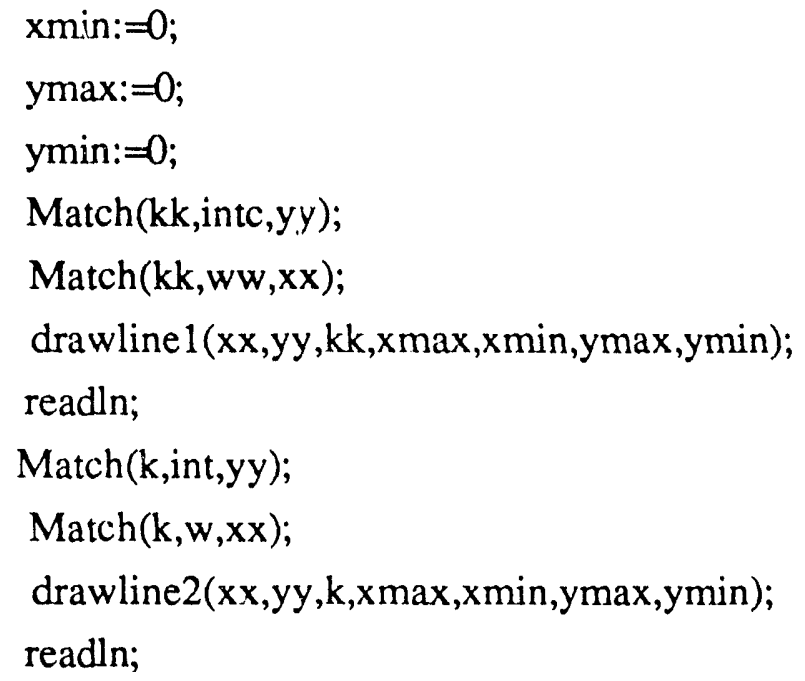

Procedure Interpolate(x,y:wave; xx:real; var yy:real; t:integer);

\{Interpolation scheme taken from Bevington ${ }^{3.9}$, eq. 13-11 and 13-12\}

var

i:integer,

$\mathrm{d} 2, \mathrm{~d} 3, \mathrm{~d} 4, \mathrm{~d}, \mathrm{a} 1, \mathrm{a} 2, \mathrm{a} 3, \mathrm{a} 4$, sum:double;

Begin

for $i:=1$ to 4 do

begin

$x[i]:=\ln (x[i])$;

$y[\mathrm{i}]:=\ln (\mathrm{y}[\mathrm{i}])$;

end;

$\mathrm{xx}:=\ln (\mathrm{xx})$;

$y y:=\ln (y y)$;

$\mathrm{d}:=(\mathrm{xx}-\mathrm{x}[1]) /(\mathrm{x}[2]-\mathrm{x}[1])$;

$\mathrm{d} 2:=(\mathrm{x}[2]-\mathrm{x}[1]) /(\mathrm{x}[2]-\mathrm{x}[1])$;

$\mathrm{d} 3:=(\mathrm{x}[3]-\mathrm{x}[1]) /(\mathrm{x}[2]-\mathrm{x}[1])$;

$\mathrm{d} 4:=(\mathrm{x}[4]-\mathrm{x}[1]) /(\mathrm{x}[2]-\mathrm{x}[1])$;

a1:=y[1];

a2: $=(y[2]-a 1) / d 2$;

a3:=(y[3]-a $2 * d 3-a 1) /(d 3 *(d 3-d 2))$; 
$\mathrm{a} 4:=\mathrm{y}[4]-\mathrm{a} 3 * \mathrm{~d} 4 *(\mathrm{~d} 4-\mathrm{d} 2)-\mathrm{a} 2 * \mathrm{~d} 4-\mathrm{a} 1$

$\mathrm{a} 4:=\mathrm{a} 4 /(\mathrm{d} 4 *(\mathrm{~d} 4-\mathrm{d} 2) *(\mathrm{~d} 4-\mathrm{d} 3))$;

$y y:=a 1+a 2 * d+a 3 * d *(d-d 2)+a 4 * d *(d-d 2) *(d-d 3) ;$

yy:=exp(yy);

End; \{of Interpolation \}

$\{* * * * * * * * * * * * * * * * * * * * * * * * * * * * * * * * * * * * * * * * * * * * * * * * * * * * * * * * * * * * * * * *\}$

Begin \{Main

\{Number of points at which we need to calculate the intensity

$\mathrm{kk}:=51$;

\{Number of points at which theoretical values are available\}

$\mathrm{k}:=2.1$;

\{Read wavelengths $c^{\text {t }}$ which we need to calculate the intensity\}

reset(a,'w.dat');

for $\mathrm{i}:=1$ to $\mathrm{kk}$ do

$\operatorname{readln}(\mathrm{a}, \mathrm{ww}[\mathrm{i}])$;

close(a);

\{Read theoretical values of intensity\}

reset(a,'i6.dat'); $\left\{^{*}\right\}$

for $i:=1$ to $k$ do

readln(a, int [i]);

close(a);

\{Read wavelengths at which theoretical intensities are avalable $\}$

reset(a,'16.dat'); (*) $^{*}$

for $i:=1$ to $k$ do

$\operatorname{readln}(\mathrm{a}, \mathrm{w}[\mathrm{i}])$;

close(a);

Rewrite(b,'intc6.dat'); $\quad$ * $^{*}$

(Read index for interpolation. Index set such that interpolated points

lie in between two middle points of 'nose selected\}

reset(a,'p6.dat'); * $\}^{*}$

for $\mathrm{i}:=1$ to $\mathrm{kk}$ do

readln(a,p[i]);

close(a); 


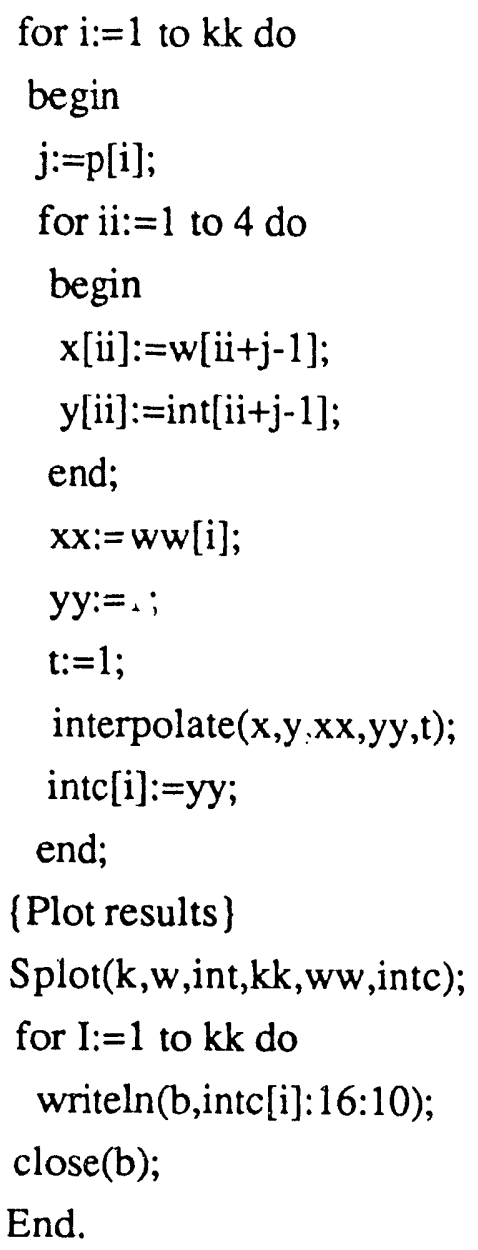

Program H_beta_curve_Interp;

(This program generates theoretical $\mathrm{H}$ beta intensity vs wavelength curves at equal spacing between the points given in Ref.3.1 at the delta wavelengths in w.dat. It generates the curves between ne $=3.16 \mathrm{e} 20$ and $3.16 \mathrm{e} 21 \mathrm{~m}-3$.

\{\$U Graph2D\}

$\{\$ R+, I-\}$

uses Memtypes, Quickdraw, Graph2D;

type

wave = array[1..51] of real;

lwave = array[1..17] of real; 
dwave =array $[1 . .17,1 . .51]$ of real;

file_name $=$ string[20];

$\mathrm{ff}=\operatorname{array}[1 . .17]$ of file_name;

var

$\mathrm{i}, \mathrm{j}, \mathrm{k}, \mathrm{kk}, \mathrm{ii}, \mathrm{iii}$ :integer;

a,b:text;

int, $w$, intc, $w w, x, y$, intc 1 , intc2, intc3, intc4, intc5, intc6: wave;

nne:array[1..6] of real;

ne:lwave;

iintc:dwave;

p:array[1..51] of integer;

$\mathrm{xx}, \mathrm{yy}, \mathrm{t}:$ real;

name:file_name;

$\{* * *$

Procedure Match (k:integer; yy:wave; var $x x: a r) ;$

\{See Match routine above\}

end; \{Procedure Match\}

$\{* * * * * * * * * * * * * * * * * * * * * * * * * * * * * * * * * * * * * * * * * * * * * * * * * * * * * * * * * * * * * * * *\}$

Procedure Norm(k,kk:integer; w:wave; var intc:dwave);

var

$\mathrm{i}, \mathrm{ii}, \mathrm{jj}:$ integer;

max,sum1:real;

begin

for $\mathrm{i}:=1$ to $\mathrm{kk}$ do begin

sum $1:=0$;

for $\mathrm{j}:=2$ to $\mathrm{k}$ do begin

sum $1:=\operatorname{sum} 1+((\operatorname{iintc}[\mathrm{i}, \mathrm{j}]+\mathrm{iintc}[\mathrm{i}, \mathrm{j}-1]) / 2) *(w[j]-w[j-1])$;

for $\mathrm{j}:=1$ to $\mathrm{k}$ do

iintc $[i, j]:=i i n t c[i, j] /$ sum 1 ;

end;

end; \{of Norm \}

$\{* * * * * * * * * * * * * * * * * * * * * * * * * * * * * * * * * * * * * * * * * * * * * * * * * * * * * * * * * * * * * * * *\}$

Procedure Splot (k:integer; w:wave;iintc:dwave); 
\{Plot using graghics package Graph2D)

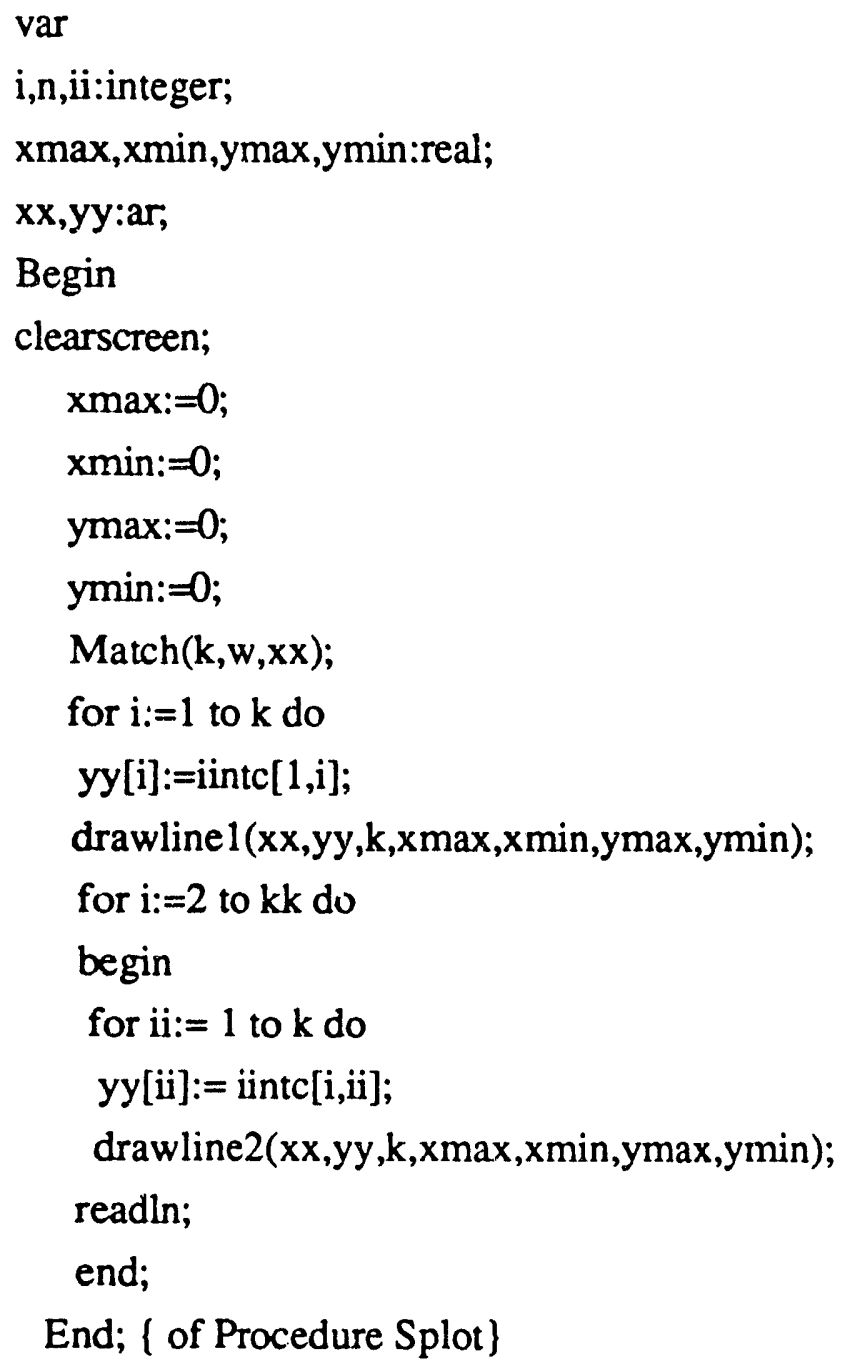

Procedure Interpolate(x,y:wave; $x x: r e a l ;$ var yy:real);

\{see above interpolation routine\}

End; \{of Interpolation \}

$\{* * * * * * * * * * * * * * * * * * * * * * * * * * * * * * * * * * * * * * * * * * * * * * * * * * * * * * * * * * * * * * * *\}$

Procedure look(call:file_name;k:integer; var intc:wave);

var

I:integer,

c:text;

begin 


$$
\begin{aligned}
& \text { reset(c,call); } \\
& \text { for } \mathrm{i}:=1 \text { to } \mathrm{k} \text { do } \\
& \text { readln(c,intc[i]); } \\
& \text { close(c); } \\
& \text { end; }\{\text { of look }\}
\end{aligned}
$$

$\{* * * * * * * * * * * * * * * * * * * * * * * * * * * * * * * * * * * * * * * * * * * * * * * * * * * * * * * * * * * * * * * *\}$

Procedure writeoutput( k,kk:integer;iintc:dwave);

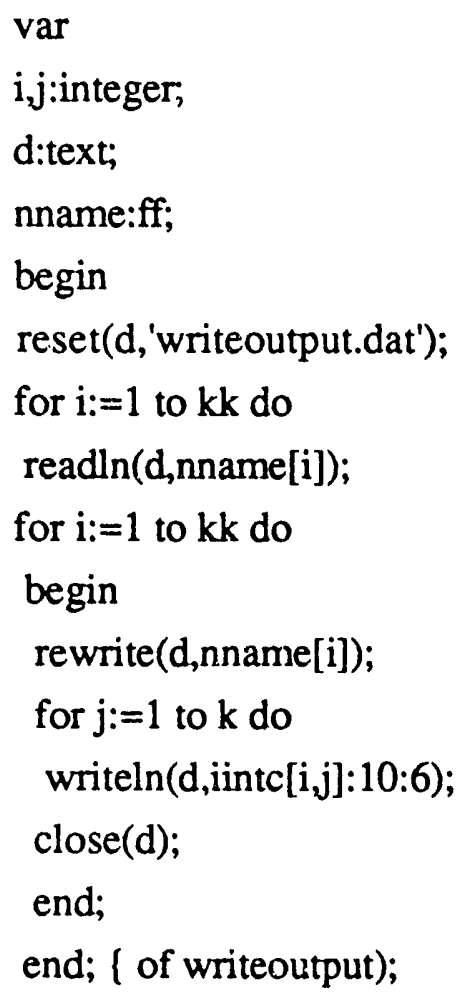

(Number of wavelength points at which we need to calculate the intensity) $\mathrm{k}:=51$;

\{Number of electron-density points at which we need to calculate profiles\} $\mathrm{kk}:=17$;

\{Read wavelengths at which we need to calculate the intensity\} reset(a,'w.dat');

for $\mathrm{i}:=1$ to $\mathrm{k}$ do $\operatorname{readln}(a, w[i])$; 
close(a);

[Read theoretical values of intensity at tabulated theoretical values]

name:='intc1.dat';

look(name,k,intc1);

name:='intc2.dat';

look(name,k,intc2);

name:='intc3.dat';

look(name,k,intc3);

name:='intc4.dat';

look(name,k,intc4);

name:='intc5.dat';

look(name,k,intc5);

name:='intc6.dat';

look(name,k,intc6);

[Assign theoretical ne values at which profiles are available]

$\mathrm{t}:=\exp ((1 / 16) * \ln (10))$

ne[1]:=10;

for $\mathrm{i}:=2$ to $\mathrm{kk}$ do

begin

ne[i]:=ne[i-1]*t;

end;

(Write theoretical ne values at which profiles are available)

nne[1]:=3.162;

nne[2]: $=10$;

nne[3]:=nne[1]*10;

nne[4]:=nne[2]*10;

nne[5]:=nne[3]*10;

nne[6]:=nne[4]*10;

\{Calculate the interpolated values of intensity at these electron densities\}

for $i:=1$ to $k$ do

begin

iintc[1,i]:=intc2[i];

iintc $[9, \mathrm{i}]:=$ intc $3[\mathrm{i}]$; 


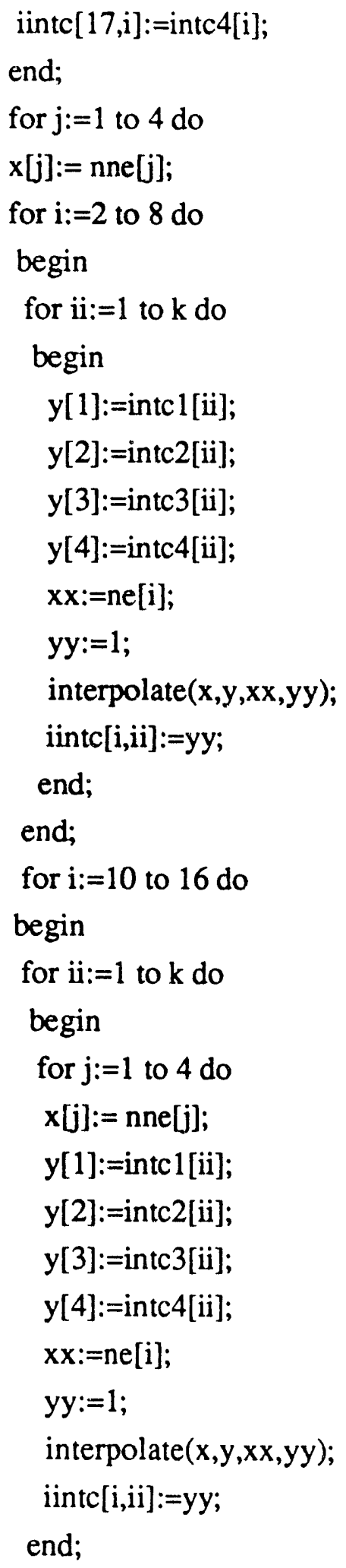


end;

$\operatorname{Norm}(k, k k, w, i i n t c)$;

rewrite(b,'results.dat');

for $i:=1$ to $k$ do

begin

write(b,w[i],' ');

for $\mathrm{j}:=1$ to $\mathrm{kk}$ do

write(b,iintc[j,i]:10:8,' ');

writeln(b);

end;

close(b);

readln;

writeoutput(k,kk,iintc);

\{Plot results\}

Splot(k,w,iintc);

End.

Program H_Beta_Profile;

(This program reads the red and blue wings of the $H$ beta profile from the hydrogen lamp, averages them, and then computes the intensities at the wavelengths necessary for convolution. The output is area-normalized.\}

\{\$U Graph2D\}

$\{\$ I-, \mathrm{r}+\}$

uses Memtypes, Quickdraw, Graph2D;

type

wave $=\operatorname{array}[1 . .150]$ of real;

inten $=\operatorname{array}[1 . .512]$ of real;

var

$\mathrm{i}, \mathrm{j}, \mathrm{k}, \mathrm{kk}, \mathrm{ii}, \mathrm{t}:$ integer;

a,b,c:text;

wint,wpix,s1,s2,s, intc, $x, y:$ wave;

p:array[1..52] of integer;

$\mathrm{xx}, \mathrm{yy}, \mathrm{d}, \mathrm{la}$, sum:real; 
$\mathrm{y} 1, \mathrm{y} 2, \mathrm{x} 1, \mathrm{x} 2$,wa:wave;

$\mathrm{u}, \mathrm{v}, \mathrm{r}:$ inten;

$\{* * * * * * * * * * * * * * * * * * * * * * * * * * * * * * * * * * * * * * * * * * * * * * * * * * * * * * * * * * * * * * * *\}$

Procedure Norm(k:integer, wint:wave; var x:wave);

\{This procedure normalizes the instrumental data by the total area under the Hbeta curve)

var

i:integer,

sum1:real;

begin

sum $1:=0$;

for $i:=2$ to $k$ do begin

$\operatorname{sum} 1:=\operatorname{sum} 1+((x[\mathrm{i}]+x[\mathrm{i}-1]) / 2) *(\operatorname{wint}[\mathrm{i}]-\operatorname{wint}[\mathrm{i}-1])$;

end;

writeln('sum ',sum1:8:7);

readln;

for $\mathrm{i}:=1$ to $\mathrm{k}$ do

$x[i]:=x[i] /$ sum 1 ;

end; \{of Norm \}

$\{* * * * * * * * * * * * * * * * * * * * * * * * * * * * * * * * * * * * * * * * * * * * * * * * * * * * * * * * * * * * * * * *\}$

Procedure Match (k:integer; yy:wave; var xx:ar);

\{see above for Match routine\}

end; \{Procedure Match\}

$\{* * * * * * * * * * * * * * * * * * * * * * * * * * * * * * * * * * * * * * * * * * * * * * * * * * * * * * * * * * * * * * * *\}$

Procedure Splot (k:integer; w,int:wave; kk:integer; ww,intc:wave);

\{see above for splot routine\}

End; \{ of Procedure Splot \}

$\{* * * * * * * * * * * * * * * * * * * * * * * * * * * * * * * * * * * * * * * * * * * * * * * * * * * * * * * * * * * * * * * * *)\}$

Procedure Interpolate(i:Integer,x,y:wave; xx:real; var yy:real);

\{see above for interpolation routine\}

End; \{of Interpolation \}

$\{* * * * * * * * * * * * * * * * * * * * * * * * * * * * * * * * * * * * * * * * * * * * * * * * * * * * * * * * * * * * * * * *\}$

Begin \{Main\} 
\{Number of points at which we need to calculate the intensity\}

$\mathrm{kk}:=51$;

\{Number of points at which experimental values are available\}

$\mathrm{k}:=86$;

\{Wavelength per pixel\}

$\mathrm{d}:=0.616$;

\{Read wavelengths at which we need to calculate the interpolated intensities\}

reset(a,'w.dat');

for $\mathrm{i}:=1$ to $\mathrm{kk}$ do

readln(a,wint[i]);

close(a);

\{Create the delta lambdas from the center of the $\mathrm{H}$ beta line

corresponding to the intensities in $\mathrm{s} 1$ and $\mathrm{s} 2$ \}

wpix[1]: $=0.01$;

for $i:=1$ to $k+1$ do

wpix $[\mathrm{i}+1]:=(\mathrm{d} / 2)+(\mathrm{i}-1) * \mathrm{~d} ;$

\{Read experimental profile of hydrogen beta\}

\{Skip the unused portion of the data\}

reset(a,'c4exp.dat');

reset(b,'res.dat');

for $i:=1$ to 554 do

begin

readln $(\mathrm{a}, \mathrm{u}[\mathrm{i}])$;

$\mathrm{u}[\mathrm{i}]:=\mathrm{u}[\mathrm{i}]-82$;

end;

(Read the response function data up to pixel 510. The remaining points are assigned using fourth order polynomial curve fit to the first 510 points.)

for $\mathrm{i}:=1$ to 510 do

readln(b,v[i]);

for $\mathrm{i}:=511$ to 555 do

$v[\mathrm{i}]:=1.9012-7.8056 \mathrm{e}-3 *_{\mathrm{i}}+2.5258 \mathrm{e}-5 *_{\mathrm{i}} *_{\mathrm{i}}-4.1031 \mathrm{e}-8 *_{\mathrm{i}} *_{\mathrm{i}} *_{\mathrm{i}}+2.9417 \mathrm{e}-$

$11 *{ }^{2} * \mathrm{i} * \mathrm{i} * \mathrm{i}$

for $i:=1$ to 555 do 


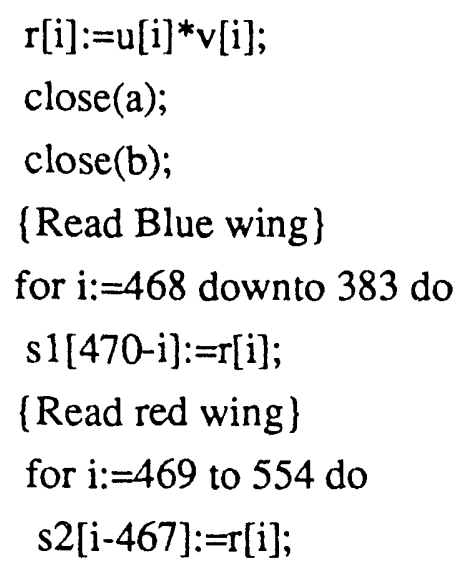

[Average the blue and red wing intensities\}

for $\mathrm{i}:=2$ to $\mathrm{k}$ do

begin

$\mathrm{s}[\mathrm{i}]:=0.5 *(\mathrm{~s} 1[\mathrm{i}]+\mathrm{s} 2[\mathrm{i}])$;

end;

$\{$ Calculate intensity at delta lambda $=0$ \}

for $\mathrm{ii}:=1$ to $4 \mathrm{do}$

begin

$\mathrm{x}[\mathrm{ii}]:=$ wpix $[\mathrm{ii}+1]$;

$y[i i]:=s[i i+1]$;

end;

$\mathrm{xx}:=0.01$;

yy:=1;

$\mathrm{i}:=1$;

interpolate $(\mathrm{i}, \mathrm{x}, \mathrm{y}, \mathrm{xx}, \mathrm{yy})$;

$s[1]:=y y$;

\{Normalize the wing data\}

norm(k,wpix,s);

\{cp.dat contains the index for finding the intensities corresponding to the wavelengths in w.dat

reset(a,'cr.dat');

for $\mathrm{i}:=1$ to $\mathrm{kk}$ do

$\operatorname{readln}(\mathrm{a}, \mathrm{p}[\mathrm{i}])$;

close(a); 
[Calculate intensities at specified wavelengths using interpolation \}

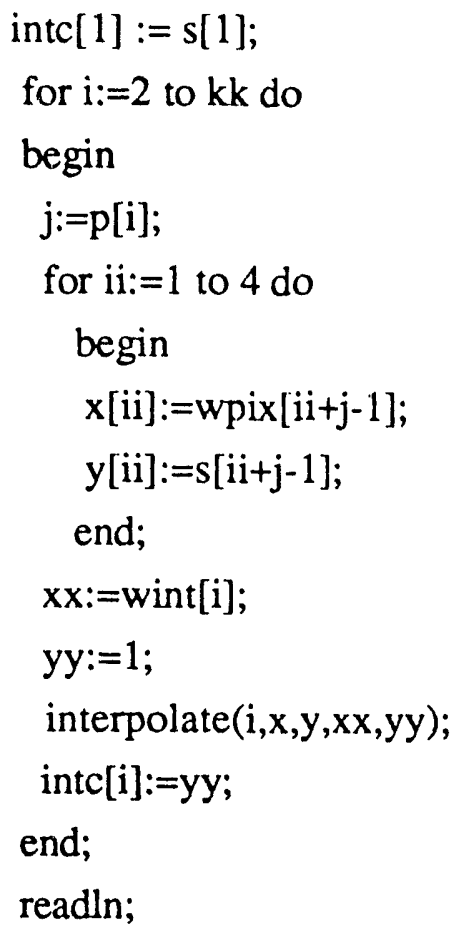

[output average wing intensities at points needed for convolution \} for $\mathrm{i}:=1$ to $\mathrm{kk}$ do $\{*\}$

$y 1[i]:=i n t c[k k-i+1]$;

ii: $=0$;

for $\mathrm{i}:=2$ to $\mathrm{kk}$ do

begin

ii: $=\mathrm{ii}+1$;

yl[kk+ii]:=intc[i];

end;

$\mathrm{ii}:=2 * \mathrm{kk}-1$;

reset(a,'wave101.dat');

for $\mathrm{i}:=1$ to ii do

readln(a,wa[i]);

close (a);

norm(ii,wa,y1);

norm(ii,wa,y1);

rewrite (a,'c44.dat'); 
for $\mathrm{i}:=1$ to $2^{*} \mathrm{kk}-1$ do begin

writeln(a,y1[i]:10:8);

end;

close(a);

Splot(10,wint,intc,43,wpix,s);

End.

Program Convolution;

\{This program is used to convolve the theoretical $\mathrm{H}$ beta profiles with the instrumental function.)

\{\$U Graph2D\}

$\{\$ I-, R+\}$

uses Memtypes, Quickdraw, Graph2D;

type

$\mathrm{t} 1$ = array [1..103] of double;

$\mathrm{t} 2=\operatorname{array}[1 . .205]$ of double;

file_name =string[20];

$\mathrm{ff}=\operatorname{array}[1 . .34]$ of file_name;

$\mathrm{t} 4=\operatorname{array}[1.34,1 . .103]$ of real;

var

i,imax,jmax,j,n,ij:integer;

$\mathrm{v}, \mathrm{xmax}, \mathrm{xmin}, \mathrm{ymax}, \mathrm{ymin}, \max$,averag,sigma:double;

$\mathrm{x}, \mathrm{xx}, \mathrm{y}, \mathrm{zz}$, lam,w:t 1 ;

inname,outname:ff;

a,c,d,e,aa,bb:text;

out: $t 4$;

$\{* * * * * * * * * * * * * * * * * * * * * * * * * * * * * * * * * * * * * * * * * * * * * * * * * * * * * * * * * * * * * * * *\}$

Procedure Match (k:integer; yy:t1; var xx:ar);

Begin

for $\mathrm{i}:=1$ to $\mathrm{k}$ do

$\mathrm{xx}[\mathrm{i}]:=\mathrm{yy}[\mathrm{i}]$; 
end; (Procedure Match)

$\{* * * * * * * * * * * * * * * * * * * * * * * * * * * * * * * * * * * * * * * * * * * * * * * * * * * * * * * * * * * * * * * *\}$

Procedure Norm(imax:integer; lam:t1; var $\mathrm{x}: \mathrm{t} 1$ );

(See above area normalization routine)

end; \{of Norm \}

$\{* *$

Procedure Splot (k:integer; $\mathrm{x}, \mathrm{y} 1, \mathrm{y} 2, \mathrm{y} 3: \mathrm{t} 1)$;

End; \{ of Procedure Splot \}

$\{* * * * * * * * * * * * * * * * * * * * * * * * * * * * * * * * * * * * * * * * * * * * * * * * * * * * * * * * * * * * * * * *\}$

Procedure Convolution (imax:integer; z2,z3:t1; var zz:t1);

(Convolution in performed by multiplying each $\mathrm{z} 1$ value by the entire $\mathrm{z} 2$ array, storing this data in an array $z 4$, and then assigning these values to the appropriate positions in another array $z 1$ so that they can be summed, giving the convolved profile in a $1 D$ array $z 1$. The array $z z$ is used so that the output array is of the same length as the input arrays. \}

var

$\mathrm{i}, \mathrm{ii}, \mathrm{j}:$ integer,

sum:real;

$\mathrm{z} 4: \mathrm{t} 1$

z1:t2;

Begin

for $\mathrm{i}:=1$ to $2 *$ imax -1 do

$z 1[i]:=0.0$;

for $i:=1$ to imax do

begin

for $j:=1$ to imax do

$z 4[j]:=z 2[i] * z 3[j]$;

for $\mathrm{j}:=1$ to imax do

$z 1[j+i-1]:=z 1[j+i-1]+z 4[j]$;

end;

$\mathrm{j}:=\operatorname{trunc}(\operatorname{imax} / 2)$;

for $\mathrm{i}:=1$ to imax do

$\mathrm{zz}[\mathrm{i}]:=\mathrm{z} 1[\mathrm{i}+\mathrm{j}]$; 
end; \{of convolution\}

$\{* * * * * * * * * * * * * * * * * * * * * * * * * * * * * * * * * * * * * * * * * * * * * * * * * * * * * * * * * * * * * * * *\}$

Begin \{Main\}

reset(aa,'input_name.dat');

reset(bb,'output_name.dat');

For $\mathrm{ij}:=1$ to 34 do

Begin

readln(aa,inname[ij]);

readln(bb,outname[ij]);

End;

close(aa);

close(bb);

imax:=101; \{lengths of data sets $\}$

reset(a,'wave101.dat');

reset(d,'c44.dat');

\{averag: $=0.0$;

sigma: $=4.0 ;\}$

for $i:=1$ to imax do

begin;

readln $(\mathrm{a}, \operatorname{lam}[\mathrm{i}])$;

readln $(\mathrm{d}, \mathrm{y}[\mathrm{i}])$;

(This section of code can be used to generate a Gaussian curve

to replace the instrument response function.\}

( v:=(lam[i]-averag)/sigma;

$y[\mathrm{i}]:=0.3989422804 /$ sigma* $^{*} \exp (-\operatorname{sqr}(\mathrm{v}) / 2.0)$;

$\mathrm{x}[\mathrm{i}]:=\mathrm{y}[\mathrm{i}] ;\}$

end;

close(a);

close(d);

For $\mathrm{ij}:=1$ to 34 do

Begin

rewrite(e,outname[ij]);

reset(c,inname[ij]); 
Clearscreen;

$\{$ Read theoretical $\mathrm{H}$ beta profile (function of $\mathrm{Ne}$ ) \}

for $\mathrm{i}:=1$ to trunc $(\operatorname{imax} / 2)+1$ do

readln $(c, x[i])$;

close (c);

\{Create symmetric theoretical $\mathrm{H}$ beta profile data (function of $\mathrm{Ne}$ ).

rewrite(c,'d1.dat');

for $i:=1$ to trunc(imax $/ 2)+1$ do begin

writeln(c,x[trunc(imax/2+1)-i+1]:10:9);

(writeln(i:3,' ',x[trunc(imax/2+1)-i+1]:10:9);

readln; \}

end;

for $i:=2$ to trunc(imax $/ 2)+1$ do begin

writeln(c,x[i]:10:9);

( writeln(i:3,' ',x[i]:10:9);

readln; \}

end;

close(c);

reset(a,'d1.dat');

for $i:=1$ to imax do

readln $(\mathrm{a}, \mathrm{x}[\mathrm{i}])$;

close(a);

Norm(imax,lam,x);

Norm(imax,lam,x);

readln;

clearscreen;

\{Perform convolution\}

for $i:=1$ to imax do

$\mathrm{zz}[\mathrm{i}]:=0.0$;

convolution(imax, $\mathrm{x}, \mathrm{y}, \mathrm{zz}$ );

\{for $\mathrm{i}:=1$ to imax do begin

writeln(i:3,' ',x[i]:7:6,' ',y[i]:7:6,' ',zz[i]:7:6);

readln; 
end; \}

\{Plot the results\}

\{Splot(imax,lam,y,zz,x);

readln; \}

clearscreen;

reset(a,'w.dat');

for $i:=1$ to trunc $(\operatorname{imax} / 2+1)$ do begin

$x x[i]:=z z[\operatorname{trunc}(\operatorname{imax} / 2+1)-i+1]$;

readln $(a, w[i])$;

end;

close(a);

Norm(trunc(imax $/ 2+1), w, x x)$;

Norm(trunc(imax $/ 2+1), w, x x)$;

readln;

clearscreen;

for $\mathrm{i}:=1$ to trunc(imax $/ 2+1)$ do begin

$\operatorname{out}[\mathrm{ij}, \mathrm{i}]:=x \times[\mathrm{i}]$;

writeln(e,out[ij,i]:10:9);

end;

close (e);

end;

\{Write results in output file area.theoret.dat for plotting

rewrite(e,'theo.area.dat');

for $\mathrm{i}:=1$ to trunc $(\mathrm{imax} / 2+1)$ do begin

write(e,w[i]:5:4,' ');

for $\mathrm{j}:=1$ to 34 do begin

write(e,out [j,i]:10:9,' ');

end;

writeln(e);

end;

close(e);

\{Output results to output file for plotting results of convolution\}

\{rewrite(d,'convout1.dat'); 
for $i:=1$ to imax do

writeln(d,lam[i]:5:4,' ',x[i]:10:9,' ',y[i]:10:9,' ',zz[i]:10:9);

close(d);

End.

Program Wing_create;

(This program reads the red and blue wings of the $\mathrm{H}$ beta profile fromthe spectra taken from the railgun shots, averages them, and then computes the intensities at the wavelengths necessary for convolution \}

\{\$U Graph2D\}

\{\$I-,r+\}

uses Memtypes, Quickdraw, Graph2D, Sane;

type

wave $=$ array $[1 . .150]$ of real;

file_name =string[20];

$\mathrm{ff}=\mathrm{array}[1 . .20]$ of file_name;

nwave $=$ array $[1 . .17,1 . .75]$ of real;

var

$\mathrm{i}, \mathrm{j}, \mathrm{k}, \mathrm{kk}, \mathrm{ij}$ :integer,

aa,bb:text;

s1,s2,ww,u:wave;

s:nwave;

$x x, y y, d$ :real;

inname:ff;

$\{* * * * * * * * * * * * * * * * * * * * * * * * * * * * * * * * * * * * * * * * * * * * * * * * * * * * * * * * * * * * * * * *)\}$

Procedure Norm(k,ij:integer; d:real; var x:wave);

var

i:integer,

max,suml :real;

begin

$\operatorname{sum} 1:=0$ 
for $\mathrm{i}:=2$ to $\mathrm{k}$ do

$$
\text { sum } 1:=\operatorname{sum} 1+((x[i-1]+x[i]) / 2) * d ;
$$

for $\mathrm{i}:=1$ to $\mathrm{k}$ do

$\mathrm{x}[\mathrm{i}]:=\mathrm{x}[\mathrm{i}] /$ sum 1 ;

end; \{ of Norm \}

$\{* * * * * * * * * * * * * * * * * * * * * * * * * * * * * * * * * * * * * * * * * * * * * * * * * * * * * * * * * * * * * * * *\}$

Procedure Match (k:integer, yy:wave; var xx:ar);

[See Match above)

end; \{Procedure Match\}

$\{* * * * * * * * * * * * * * * * * * * * * * * * * * * * * * * * * * * * * * * * * * * * * * * * * * * * * * * * * * * * * * * * * * *\}$

Procedure Splot (k,ii:integer, w,s1,s2:wave;s:nwave);

\{See plotting routine above\}

End; \{ of Procedure Splot\}

$\{* * * * * * * * * * * * * * * * * * * * * * * * * * * * * * * * * * * * * * * * * * * * * * * * * * * * * * * * * * * * * * * *\}$

Begin \{Main\}

reset(aa,'outname.dat');

For $\mathrm{ij}:=1$ to 17 do

begin

readln(aa,inname[ij]);

end;

close(aa);

\{Number of points at which we need to calculate the intensity\}

$\mathrm{kk}:=51$;

\{Wavelength per pixel\}

$\mathrm{d}:=0.616$;

\{Number of points at which experimental values are available \}

$\mathrm{k}:=74$;

For $\mathrm{ij}:=1$ to $17 \mathrm{do}$

Begin

\{Read experimental profile of hydrogen beta\}

[Skip the unused portion of the data]

reset(bb,inname[ij]);

for $\mathrm{j}:=1$ to $510 \mathrm{do}$ 
begin

readln $(b b, u[j])$;

end;

close(bb);

\{Write red wing

for $i:=437$ to $510 \mathrm{do}$

$\mathrm{s} 2[\mathrm{i}-436]:=\mathrm{u}[\mathrm{i}]$;

\{Write Blue wing)

for $i:=436$ downto 361 do

$\mathrm{s} 1[437-\mathrm{i}]:=\mathrm{u}[\mathrm{i}]$;

reset(bb,inname[ij]);

\{Normalize the wing data by area\}

norm(k,ij,d,s1);

norm(k,ij,d,sl);

norm(k,ij,d,s2);

norm(k,ij,d,s2);

\{Average the blue and red wing intensities\}

for $i:=1$ to $k$ do

begin

$\mathrm{s}[\mathrm{ij}, \mathrm{i}]:=0.5 *(\mathrm{~s} 1[\mathrm{i}]+\mathrm{s} 2[\mathrm{i}])$;

end;

for $\mathrm{i}:=1$ to $\mathrm{k}+1$ do

$\mathrm{ww}[\mathrm{i}]:=((\mathrm{d} / 2)+(\mathrm{i}-1) * \mathrm{~d}) / 10.0$;

\{Splot(k,ij,ww,s1,s2,s);\}

end;

\{output average wing intensities.\}

rewrite(aa,'wings_area.dat');

for $i:=1$ to $k$ do

begin

write(aa,ww[i]:5:4,' ');

for $\mathrm{j}:=1$ to $17 \mathrm{do}$

write(aa,s[j,i]:10:8,' ');

writeln(aa); 
end;

close(aa);

End. 


\section{APPENDIX III \\ EFFECT OF BOUNDARY LAYERS ON TEMPERATURE DETERMINATION VIA BOLTZMANN PLOTS}

For the experiments described in Chapters 3 and 4 spectral data was acquired through the glass side walls of the railgun. The characterization of the plasma armatures in these Chapters assumed a uniform plasma distribution along the line of sight. However, there are actually cooler boundary layers along the glass side-walls, and the temperature in the plasma core may also be non-uniform. Hence, the temperatures determined in these chapters was averaged along the line-of-sight. The effects of this averaging are examined here.

\section{Case I}

First, let us assume that the plasma temperature within the railgun was uniform except for thin boundary layers at the glass side-walls. Divide the plasma cross-section in the direction of observation into eleven cells of equal length and assume that PLTE exists in these cells, i. e., the excited state populations of $\mathrm{O}^{+}$ considered are in thermal equilibrium with electrons and that the plasma is optically thin. If total line intensities $S_{1}$ and $S_{2}$ are observed at wavelengths $\lambda_{1}$ and $\lambda_{2}$ for the calculation of temperature via a Boltzmann plot, then

$$
S_{1}=S_{1}(1)+S_{1}(2)+\ldots+S_{1}(11)
$$




$$
\text { and } S_{2}=S_{2}(1)+S_{2}(2)+\ldots+S_{2}(11)
$$

where superscripts denote the cell numbers and subscripts denote wavelengths. The temperature $T^{*}$ inferred from a line-of-sight measurement is defined by

$$
\frac{S_{1}}{S_{2}} \equiv \frac{c_{1}}{c_{2}} \exp \left(-\frac{E_{1_{1}}-E_{l_{2}}}{k T^{*}}\right)
$$

where $c_{1}=\left(A_{1} g_{1} / \lambda_{1}\right), c_{2}=\left(A_{2} g_{2} / \lambda_{2}\right)$. Assume that the temperatures of $T^{(1)}$ and $\mathrm{T}^{(11)}$ are $1.5 \times 10^{4} \mathrm{~K}$ and that the rest of the cells have a temperature of $\mathrm{T}^{(2)}=3.0 \times 10^{4}$ $K$. Also assume that $E_{l_{1}}=200,000 \mathrm{~cm}^{-1}$, and $E_{l_{2}}=180,000 \mathrm{~cm}^{-1}$, typical values for $\mathrm{O}^{+}$. It follows from Eq. A3.1 that

$$
\begin{aligned}
\frac{S_{1}}{S_{2}} & =\frac{2\left(S_{1}(1)\right)+9\left(S_{1}(2)\right)}{2\left(S_{2}(1)\right)+9\left(S_{2}{ }^{(2)}\right)} \\
& =\frac{c_{1}\left[2 \exp \left(-E_{1_{1}} / \mathrm{kT}^{(1)}\right)+9 \exp \left(-\mathrm{E}_{1_{1}} / \mathrm{kT}^{(2)}\right)\right]}{c_{2}\left[2 \exp \left(-\mathrm{E}_{1_{2}} / \mathrm{kT}^{(1)}\right)+9 \exp \left(-\mathrm{E}_{\mathrm{l}_{2}} / \mathrm{kT}^{(2)}\right)\right]} \\
& =\frac{c_{1}}{c_{2}} \exp \left(-\frac{\mathrm{E}_{1_{1}}-\mathrm{E}_{1_{2}}}{\mathrm{kT} \mathrm{T}^{(2)}}\right) \frac{1+(9 / 2) \exp \left[\left(-\mathrm{E}_{1} / \mathrm{k}\right)\left(1 / \mathrm{T}^{(2)}-1 / \mathrm{T}^{(1)}\right)\right]}{1+(9 / 2) \exp \left[\left(-\mathrm{E}_{\mathrm{l}_{2}} / \mathrm{k}\right)\left(1 / \mathrm{T}^{(2)}-1 / \mathrm{T}^{(1)}\right)\right]}
\end{aligned}
$$

Inserting numerical values into A3.3 gives

$$
\begin{aligned}
\frac{S_{1}}{S_{2}} & =\frac{c_{1}}{c_{2}} \exp \left(-\frac{E_{l_{1}}-E_{l_{2}}}{k T^{(2)}}\right) \frac{1+3.3 \times 10^{-4}}{1+8.6 \times 10^{-4}} \\
& \approx \frac{c_{1}}{c_{2}} \exp \left(-\frac{E_{l_{1}}-E_{l_{2}}}{k T^{(2)}}\right) .
\end{aligned}
$$


Hence the temperature $T^{*}$ that would be inferred from the line-of-sight measurement is very close to the plasma core temperature of $3.0 \times 10^{4} \mathrm{~K}$ and is not affected by the presence of the boundary layers.

Case II

In this case assume the following temperature profile: $\mathrm{T}^{(1)}=\mathrm{T}^{(11)}=1.5 \times 10^{4}$ $\mathrm{K}, \mathrm{T}^{(2)}=\mathrm{T}^{(10)}=2.0 \times 10^{4} \mathrm{~K}, \mathrm{~T}^{(3)}=\mathrm{T}^{(9)}=2.3 \times 10^{4} \mathrm{~K}, \mathrm{~T}^{(4)}=\mathrm{T}^{(8)}=2.6 \times 10^{4} \mathrm{~K}$, $\mathrm{T}^{(5)}=\mathrm{T}^{(7)}=2.9 \times 10^{4} \mathrm{~K}$, and $\mathrm{T}^{(6)}=3.0 \times 10^{4} \mathrm{~K}$. Using Eqs. $\mathrm{A} 1$ and $\mathrm{A} 2$ and following the same procedure as in Case $\mathrm{I}$, one obtains $\mathrm{T}^{*}=2.7 \times 10^{4} \mathrm{~K}$. Note that this temperature is higher than the average temperature of $2.3 \times 10^{4} \mathrm{~K}$, calculated by weighting the temperature of each cell equally. This example shows that the temperature calculated via a Boltzmann plot is closer to the maximum temperature in the plasma because of the non-linear dependence of emission on temperature. 


\section{APPENDIX IV}

\section{ERROR ESTIMATES FOR IN-SITU TEMPERATURE CALCULATIONS}

\section{A4.1 Introduction}

The method used to estimate the uncertainties in the in-situ electron temperatures and densities of Chapter 3 is outlined in this appendix.

A4.2 Temperature Uncertainties

Using Eqs. $12-14$ of of Section 3.9 it can be shown that the electron temperature can be expressed as

$$
\mathrm{T}_{\mathrm{e}} \mathrm{3}^{3 / 2} \exp \left[-\mathrm{E}^{\prime} / \mathrm{kT}_{\mathrm{e}}\right]=\mathrm{B}\left(\mathrm{n}_{\mathrm{e}}^{2} \mathrm{~A} / \mathrm{SX}\right)
$$

where $\mathrm{B}$ is a constant, and $\mathrm{E}^{\prime}=\mathrm{E}_{\mathrm{i}}^{\mathrm{H}}-\mathrm{E}_{\mathrm{l}}^{\mathrm{H}}-\Delta \mathrm{E} . \mathrm{E}_{\mathrm{i}}^{\mathrm{H}}$ is the ionization energy, $\mathrm{E}_{\mathrm{l}}^{\mathrm{H}}$ is the upper energy level of the $\mathrm{H}_{\beta}$ transition, and $\Delta \mathrm{E}$ is the lowering of ionization energy due to plasma-particle interaction near the ionization limit. Letting the left hand side of Eq. $A 4.1=f\left(T_{e}\right)$, one can write an expression for the variance in $f$ as follows: ${ }^{3.9}$ :

$$
\sigma_{\mathrm{f}}^{2}=\sigma_{\mathrm{n}_{\mathrm{e}}}{ }^{2}\left(\partial \mathrm{f} / \partial \mathrm{n}_{\mathrm{e}}\right)^{2}+\sigma_{\mathrm{A}}^{2}(\partial \mathrm{f} / \partial \mathrm{A})^{2}+\sigma_{\mathrm{S}}^{2}(\partial \mathrm{f} / \partial \mathrm{S})^{2}+\sigma_{\mathrm{X}}{ }^{2}(\partial \mathrm{f} / \partial \mathrm{X})^{2}
$$

where $\sigma$ is the estimated error (Note that in the formulation of Ref. $3.9 \sigma$ is the standard deviation in the measurement at a single point and that we have neglected 
$\sigma_{\mathrm{n}_{\mathrm{e}}, \mathrm{A}}, \sigma_{\mathrm{n}_{\mathfrak{e}}, \mathrm{s}}$, etc., which are uncorrelated fluctuations.). Combining Eqs. A4.1 and A4.2 gives

$$
\sigma_{\mathrm{f}}^{2 / \mathrm{f}^{2}}=4 \sigma_{\mathrm{n}_{\mathrm{e}}}^{2 / \mathrm{n}_{\mathrm{e}}}{ }^{2}+\sigma_{\mathrm{A}}^{2} / \mathrm{A}^{2}+\sigma_{\mathrm{S}}^{2} / \mathrm{S}^{2}+\sigma_{\mathrm{X}}{ }^{2} / \mathrm{X}^{2}
$$

Equation A4.3 is an expression for the error in $\mathrm{f}$ which must be related back to the error in T. Recall that

$$
f=T_{e}^{3 / 2} \exp \left[-E^{\prime} / k T_{e}\right]
$$

It follows that

$$
\partial f / \partial T_{e}=\exp \left[-E^{\prime} / k T_{e}\right]\left\{1.5 T_{e} e^{1 / 2}+E^{\prime} /\left(k T_{e}{ }^{1 / 2}\right)\right\}
$$

and that

$$
\partial f / f=\left[3 / 2+E^{\prime} / k T_{e}\right] \partial T_{e} / T_{e}
$$

Assuming that $\sigma_{\zeta}=\partial \zeta$, it follows that

$$
\begin{gathered}
\sigma_{\mathrm{f}} / \mathrm{f}=\left[3 / 2+\mathrm{E}^{\prime} / \mathrm{k} \mathrm{T}_{\mathrm{e}}\right] \sigma_{\mathrm{Te}} / \mathrm{T}_{\mathrm{e}} \text {, or } \\
\sigma_{\mathrm{Te}}{ }^{2} / \mathrm{T}_{\mathrm{e}}^{2}=\left[3 / 2+\mathrm{E}^{\prime} / \mathrm{k}^{\prime} \mathrm{T}_{\mathrm{e}}\right]^{-2} \sigma_{\mathrm{f}^{2} / \mathrm{f}^{2}}
\end{gathered}
$$

Substituting Eq. A4.3 into Eq. A4.4 gives

$$
\frac{\sigma_{\mathrm{Te}^{2}}}{\mathrm{~T}_{\mathrm{e}}^{2}}=\left(\frac{3}{2}+\frac{\mathrm{E}^{\prime}}{\mathrm{kT}_{\mathrm{e}}}\right)^{-2}\left(4 \frac{\sigma^{2} \mathrm{ne}_{\mathrm{e}}}{\mathrm{ne}_{\mathrm{e}}^{2}}+\frac{\sigma^{2} \mathrm{~A}}{\mathrm{~A}^{2}}+\frac{\sigma^{2} \mathrm{~S}}{\mathrm{~S}^{2}}+\frac{\sigma^{2} \mathrm{X}}{\mathrm{X}^{2}}\right) .
$$

The uncertainties $\frac{\sigma_{\mathrm{n}_{\mathrm{e}}}}{\mathrm{n}_{\mathrm{e}}}, \frac{\sigma_{\mathrm{A}}}{\mathrm{A}}, \frac{\sigma_{\mathrm{S}}}{\mathrm{S}}$, and $\frac{\sigma_{\mathrm{X}}}{\mathrm{X}}$ were estimated to be $20 \%, 25 \%$, $10 \%$, and $40 \%$ respectively. The uncertainty in the electron density $n_{e}$ is discussed in 
Section 3.7 and the uncertainty in the Einstein A coefficient was taken from Ref. 3.1. The uncertainty in the area under the $\mathrm{H}_{\beta}$ line was estimated by visual inspection. This estimate is believed to be conservative. Because all of the uncertainties on the right hand side of Eq. A4.5 have been estimated, the only remaining aspect of the equation yet to be discussed is E'. Recall that

$$
E^{\prime}=E_{i} H-E_{l}^{H}-\Delta E \text {. }
$$

$\mathrm{E}_{\mathrm{i}} \mathrm{H}$ and $\mathrm{E}_{\mathrm{l}}^{\mathrm{H}}$ are $13.6 \mathrm{eV}$ and $12.7 \mathrm{eV}$ respectively. $\Delta \mathrm{E}$ can be estimated as follows. From Ref. 3.4

$$
\Delta \mathrm{E}=\mathrm{Z} \mathrm{e}^{2} / 4 \pi \varepsilon_{\mathrm{o}} \rho_{\mathrm{d}}
$$

where $Z=1$ for a neutral species, e is the charge of an electron, $\varepsilon_{0}$ is the permittivity of free space, and $\rho_{d}$ is the Debye radius. The Debye radius can be estimated as 3.4

$$
\rho_{d}=\left(\varepsilon_{0} k T / n_{e} e^{2}\right)^{1 / 2}
$$

For an electron density of $4 \times 10^{22} \mathrm{~m}^{-3}$ and a temperature of $25,000 \mathrm{~K}, \rho_{\mathrm{d}}=1.72 \times$ $10^{-7} \mathrm{~m}$, and it follows that $\Delta \mathrm{E}=1.33 \times 10^{-21} \mathrm{~J}$ or $.0083 \mathrm{eV}$. Therefore, changes in $\Delta \mathrm{E}$ due to varying temperatures and electron densities will be negligible compared to $E_{i}{ }^{H}$ and $E_{1}{ }^{H}$, so Eq. A4.5 can be solved directly as

$$
\begin{gathered}
\sigma_{\mathrm{Te}}=\mathrm{T}_{\mathrm{e}}\left(\frac{3}{2}+\frac{\mathrm{E}_{\mathrm{i}} \mathrm{H}-\mathrm{E}_{1} \mathrm{H}}{k T_{\mathrm{e}}}\right)^{-1}\left(4 \frac{\sigma^{2} \mathrm{n}_{\mathrm{e}}}{\mathrm{n}_{\mathrm{e}}^{2}}+\frac{\sigma^{2} \mathrm{~A}}{\mathrm{~A}^{2}}+\frac{\sigma^{2} \mathrm{~S}}{\mathrm{~S}^{2}}+\frac{\sigma^{2} \mathrm{X}}{\mathrm{X}^{2}}\right)^{1 / 2}, \\
\text { or } \sigma_{\mathrm{Te}}=\mathrm{T}_{\mathrm{e}}\left(\frac{3}{2}+\frac{10000}{\mathrm{~T}_{\mathrm{e}}}\right)^{-1}\left(4 \frac{\sigma^{2} \mathrm{n}_{\mathrm{e}}}{\mathrm{n}_{\mathrm{e}}^{2}}+\frac{\sigma^{2} \mathrm{~A}}{\mathrm{~A}^{2}}+\frac{\sigma^{2} \mathrm{~S}}{\mathrm{~S}^{2}}+\frac{\sigma^{2} \mathrm{X}}{\mathrm{X}^{2}}\right)^{1 / 2},
\end{gathered}
$$

Inserting the uncertainties into Eq. A4.7 gives 


$$
\sigma_{\mathrm{Te}}=0.522 \mathrm{~T}_{\mathrm{e}}\left(\frac{3}{2}+\frac{10000}{\mathrm{~T}_{\mathrm{e}}}\right)^{-1}
$$

Using Eq. A4.8, the total estimated uncertainties in the in-situ temperatures were calculated. Table A4.1 below lists the uncertainties in the Chapter 3 temperatures calculated using Eq. A4.8. These are the uncertainties shown in Figs. 3.14 and 3.15.

Table A4.1: Uncertainties in In-Situ Temperature Calculations

$\begin{array}{ccc}\text { Time } & \begin{array}{c}\text { Temperature } \\ \left(/ 10^{3} \mathrm{~K}\right)\end{array} & \begin{array}{c}\sigma_{\mathrm{Te}} \\ \left(/ 10^{3} \mathrm{~K}\right)\end{array} \\ \mathrm{E} & 6.5 & 1.3 \\ \mathrm{~F} & 19 & 5.9 \\ \mathrm{G} & 9.3 & 2.2 \\ \mathrm{H} & 11.2 & 2.9\end{array}$

\section{A4.3 Electron Density Uncertainties}

Solving Eq. A4.1 for electron density $\left(\mathrm{n}_{\mathrm{e}}\right)$ and following the above outline, the uncertainty in $n_{e}$ can be written as

$$
\sigma_{\mathrm{ne}}=\frac{\mathrm{n}_{\mathrm{e}}}{4}\left(\frac{\sigma^{2} \mathrm{~S}}{\mathrm{~S}^{2}}+\frac{\sigma^{2} \mathrm{X}}{\mathrm{X}^{2}}+\frac{\sigma^{2} \mathrm{~A}}{\mathrm{~A}^{2}}+\frac{\sigma^{2} \mathrm{Te}}{\mathrm{T}_{\mathrm{e}}^{2}}\left(\frac{3}{2}+\frac{\mathrm{E}_{\mathrm{i}}^{\mathrm{H}}-\mathrm{E}_{\mathrm{l}}^{\mathrm{H}}}{\mathrm{kT} \mathrm{T}_{\mathrm{e}}}\right)^{2}\right)^{1 / 2}
$$

Using the temperatures from the Boltzmann plots and assuming an uncertainty in these temperatures of $20 \%$, Eq. A4.9 was used to estimate the uncertainties in the 
electron densities estimated using the absulute calibration constant at times A-C (see Table A4.2).

Table A4.2: Uncertainties in In-Situ Electron Density Calculations

$\begin{array}{cccc}\text { Time } & \begin{array}{c}\text { Temperature } \\ \left(/ 10^{3} \mathrm{~K}\right)\end{array} & \begin{array}{c}\text { Electron Density } \\ \left(/ 10^{22} \mathrm{~m}^{-3}\right)\end{array} & \begin{array}{c}\sigma_{\mathrm{Te}} \\ \left(/ 10^{3} \mathrm{~K}\right)\end{array} \\ \mathrm{A} & 20.9 & 2.1 & 0.40 \\ \mathrm{~B} & 30.8 & 3.2 & 0.68 \\ \mathrm{C} & 19.4 & 3.8 & 0.72\end{array}$




\section{APPENDIX V}

\section{FEASIBILITY ANALYSIS OF TWO-PHOTON, LASER-INDUCED FLUORESCENCE STUDIES OF RAILGUN-GENERATED, HYDROGEN PLASMAS}

The following calculations demonstrate the feasibility of using an intensified 2-D CCD camera (e.g. Princeton Instruments ICCD-576S/B) to detect the signal from a two-photon excitation of the hydrogen plasma generated by the railgun.

To determine the detectable fluorescence signal $(H$ transition from $3 D \rightarrow 2 P$ ) we must know/calculate the quenching rate, photoionization rate, and 2-photon excitation rate. Because quenching cross-sections are not very well known the principle of detailed balancing will be used to calculate them. This principle requires that for a system at equilibrium, each individual molecular process and its inverse proceed, on the average, at the same rate. ${ }^{A 5.1}$ Using this principle one can, in theory, calculate the de-excitation cross-section for electron-atom impact if one knows the excitation cross-section for the inverse process. Because the excitation cross-section $\left(\sigma_{13}\right)$ is a function of the kinetic energy (KE) of the electron-atom system, the first step is to calculate the $\mathrm{KE}$ of the exciting electrons. By conservation of energy

$$
E_{1}=E_{3}+E_{13}
$$


where $E_{3}$ and $E_{1}$ are the system KE's before and after collision and $E_{13}$ is the internal excitation energy. $E_{13}$ can be calculated using the Balmer equation

$$
\begin{gathered}
E_{13}=E_{3_{B}}-E_{1_{B}} \\
E_{13}=E_{\mathrm{c}}\left(\frac{1}{n_{3}^{2}}\right)-E_{\mathrm{c}}\left(\frac{1}{n_{1}^{2}}\right)
\end{gathered}
$$

where $E_{\mathrm{c}}$ is the energy required for excitation of the atom from ground state to the continuum. For hydrogen $E_{\mathrm{c}}=-13.6 \mathrm{eV}$, so

$$
\begin{aligned}
& E_{13}=-13.6\left(\frac{1}{3^{2}}-\frac{1}{1^{2}}\right) \\
& E_{13}=12.1 \mathrm{eV}
\end{aligned}
$$

The electron temperature in railgun plasmas can be estimated from experimental data. A5.2 These data indicate that the railgun plasma is in partial local thermodynamic equilibrium (PLTE) with excited state populations determined by the electron temperature. This temperature is somewhat greater than the heavy particle kinetic temperature. This fact allows us to calculate the $\mathrm{KE}$ at state 3 , leaving $E_{1}$ as the only unknown in Eq. 1. Rewriting Eq. 1 as

$$
E_{1}=\frac{1}{2} \mathrm{~m}^{*} \bar{g}^{2}+E_{13}
$$

and assuming that

where

$$
\begin{gathered}
\frac{1}{2} \mathrm{~m}^{*} \bar{g}^{2}=\frac{3}{2} k T_{e}, \\
m^{*}=\frac{m_{\text {atom }}+m_{\mathrm{e}}}{m_{\text {atom }} * m_{\mathrm{e}}} \cong m_{\mathrm{e}},
\end{gathered}
$$


$\mathrm{g}$ is the mean relative speed, $k$ is Boltzmann's constant, and $T_{e}$ is the electron temperature. Substituting into Eq. 1 we get :A5.3

$$
E_{1}=\frac{3}{2} k T_{e}+E_{13}
$$

Assuming an electron temperature of $25,000 \mathrm{~K}$, Eq. 3 gives:

$$
\begin{aligned}
& E_{1}=\frac{3}{2}\left(1.38 \times 10^{-23} \mathrm{~J} / \mathrm{K}\right)\left(\frac{\mathrm{eV}}{1.6 \times 10^{-19} \mathrm{~J}}\right)\left(2.5 \times 10^{-4} \mathrm{~K}\right)+12.1 \mathrm{eV} \\
& E_{1}=15.3 \mathrm{eV} .
\end{aligned}
$$

So, the excitation cross-section $\sigma_{13}$ must be obtained ior a relative $\mathrm{KE}$ of $15.3 \mathrm{eV}$ between the neutral, ground state atoms and the electrons. From Calloway, A5.4 $\sigma_{13}\left(\pi a_{0}^{2}\right)=1.0$ at $E=15.3 \mathrm{eV}$, where $a_{0}$ is the Bohr radius. Thus,

$$
\sigma_{13}=1.0 \pi\left(0.53^{\circ} \mathrm{A}\right)^{2}=0.88^{\circ} A^{2}=8.8 \times 10^{-21} \mathrm{~m}^{2}
$$

Using the same logic, $\sigma_{23}$ will be obtained. First, calculate the excitation energy $E_{23}$, then use conservation of energy between states 2 and 3 to get $E_{2}$, which yields $\sigma_{23}$

$$
\begin{aligned}
& E_{23}=-13.6\left(\frac{1}{3^{2}}-\frac{1}{2^{2}}\right)=1.89 \mathrm{eV} \\
& E_{2}=E_{3}+E_{23}=3.23+1.89=5.12 \mathrm{eV}=0.377 R y
\end{aligned}
$$

At an impact energy $E_{2}=0.38 R y$ the total excitation cross-section for the $2_{p o-3}$ excitation is $\sigma_{2 p o-3}=74.2 \pi a_{0}^{2}$. A5.5 At the same impact energy the $2 s-3$ excitation cross-section is $\sigma_{2 s-3}=6.41 \pi a_{0}^{2}$. A5.6 Thus, the total excitation cross-section from state 2 to state 3 is 


$$
\begin{aligned}
\sigma_{23} & =\sigma_{2 s-3}+\sigma_{2 p_{n-3}} \\
& =1.38 .3 \pi a_{0}^{2} \\
\sigma_{23} & =1.22 \times 10^{-18} \mathrm{~m}^{2} .
\end{aligned}
$$

Now, using detailed balancing we can calculate the de-excitation cross-sections. For transition 1-3, detailed balancing gives

$$
g_{3} E_{3} \sigma_{31}=g_{1} E_{1} \sigma_{13} \text {, }
$$

where $g_{\mathrm{n}}$ is the degeneracy of energy level and $E_{\mathrm{n}}$ is the kinetic energy of the

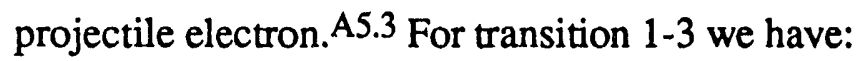

$$
\begin{aligned}
g_{1} & =2(1)^{2}=2 \\
g_{2} & =2(3)^{2}=18 \\
E_{1} & =15.3 \mathrm{eV} \\
E_{3} & =\frac{3}{2} k T=3.2 \mathrm{eV} \\
\sigma_{31} & =\frac{g_{1}}{g_{3}} \frac{E_{1}}{E_{3}} \sigma_{13} \\
& =\left(\frac{2}{18}\right)\left(\frac{15.3}{3.2}\right)\left(8.8 \times 10^{-21}\right) \\
\sigma_{31} & =4.67 \times 10^{-21} \mathrm{~m}^{2} .
\end{aligned}
$$

Similarly, for transitions $2-3$

$$
\begin{gathered}
g_{3} E_{3} \sigma_{32}=g_{2} E_{2} \sigma_{23} . \\
\sigma_{32}=\frac{g_{2} E_{2} \sigma_{23}}{g_{3} E_{3}} \\
E_{2}=5.12 \mathrm{eV}
\end{gathered}
$$




$$
\begin{gathered}
g_{2}=2(2)^{2}=8 \\
\sigma_{32}=\left(\frac{8}{18}\right)\left(\frac{5.12}{3.2}\right)\left(1.22 \times 10^{-18}\right) \\
\sigma_{32}=8.67 \times 10^{-19} \mathrm{~m}^{2} .
\end{gathered}
$$

Next we need to calculate the quenching rate. It is given by

$$
Q=n_{e} \sigma \bar{g}_{3},
$$

where $n_{e}$ is the number of electrons, $\sigma$ is the quenching cross-section, and $\bar{g}_{3}$ is the mean relative velocity between the electrons and the state 3 atoms.

$$
\begin{aligned}
\bar{g}_{3} & =\left(\frac{8 k T}{\pi m_{e}}\right)^{\frac{1}{2}} \\
& =\left(\frac{8\left(1.38 \times 10^{-2}\right)\left(2.5 \times 10^{4}\right)}{\pi\left(9.1 \times 10^{-31}\right)}\right)^{\frac{1}{2}} \\
\bar{g}_{3} & =9.8 \times 10^{5} \mathrm{~m} / \mathrm{s} .
\end{aligned}
$$

Assuming a uniform plasma distribution, the number density is calculated as follows:

$$
\begin{gathered}
n_{\mathrm{H}_{\mathrm{atoms}}}=\frac{N_{H}}{V}=\frac{1.3 \times 10^{19}}{3 \times .025 \times .075} \\
n_{H_{\text {stoms }}}=2.3 \times 10^{21} \mathrm{~m}^{-3},
\end{gathered}
$$

where the volume was assumed for a 1 meter long, $2.5 \times 7.5 \mathrm{~cm}$ cross section railgun bore. The length was multiplied by three based on the velocity and the time which the phototransistors detected an optical signal. Assuming the combined ionization of $\mathrm{H}, \mathrm{O}, \mathrm{Si}$, and $\mathrm{Cu}$ is equal to the number density of $\mathrm{H}$ atoms, let 


$$
n_{e}=n_{H}=2.3 \times 10^{21} \mathrm{~m}^{-3}
$$

Substituting the above values into Eq. 4 gives

$$
\begin{aligned}
Q_{32} & =n_{e} \sigma_{32} \bar{g}_{3} \\
& =\left(2.3 \times 10^{21}\right)\left(8.67 \times 10^{-19}\right)\left(9.8 \times 10^{5}\right) \\
Q_{32} & =1.95 \times 10^{9} s^{-1} .
\end{aligned}
$$

Similarly,

$$
\begin{aligned}
Q_{31} & =n_{e} \sigma_{31} \bar{g}_{3} \\
& =\left(2.3 \times 10^{21}\right)\left(4.67 \times 10^{-21}\right)\left(9.8 \times 10^{5}\right) \\
Q_{31} & =1.05 \times 10^{7} s^{-1}
\end{aligned}
$$

The next step is to calculate the excitation rate. From Eckbreth $\mathrm{A5.7}$

$$
W_{13}=\frac{\alpha_{13} I_{1}^{2}}{h v_{1}},
$$

where $\alpha_{13}$ is the 2-photon excitation cross-section, $I$ is the laser intensity, $h$ is Planck's constant and $v \mid$ is the laser frequency. The laser intensity can be expressed as

$$
I_{1}=\frac{E}{A t}
$$

Assuming that a laser energy of $2 \mathrm{~mJ}$ can be achieved at $205 \mathrm{~nm}$ (Bokor et al.), that the focal spot size is $10^{-3} \mathrm{~cm}^{2}$, and that the laser pulse duration is $5 \mathrm{~ns} A 5.8$, we get 


$$
\begin{aligned}
& I_{1}=\frac{2 \times 10^{-3}}{\left(10^{-3}\right)\left(5 \times 10^{-9}\right)} \\
& I_{1}=4 \times 10^{8} \frac{\mathrm{W}}{\mathrm{cm}^{2}}
\end{aligned}
$$

From Bischel ${ }^{\mathrm{A} 5.9}$ the 2-photon excitation cross-section is estimated to be $\alpha_{13}=0.5$ $x 10^{-27} \mathrm{~cm}^{4} / W$. The frequence of a laser operating at $205 \mathrm{~nm}$ is

$$
v_{1}=\frac{c}{\lambda}=\frac{3 \times 10^{8}}{205 \times 10^{-9}}=1.46 \times 10^{15} s^{-1}
$$

Substituting these values into Eq. 6 gives

$$
\begin{aligned}
W_{13} & =\frac{\alpha_{13} I_{I}^{2}}{h v_{1}} \\
& =\frac{\left(0.5 \times 10^{-27} \frac{\mathrm{cm}^{4}}{W}\right)\left(4 \times 10^{8} \frac{\mathrm{W}}{\mathrm{cm}^{2}}\right)^{2}}{\left(6.63 \times 10^{-34} \mathrm{~J} \cdot \mathrm{s}\right)\left(1.46 \times 10^{15} \mathrm{~s}^{-1}\right)} \\
W_{13} & =8.3 \times 10^{7} \mathrm{~s}^{-1}
\end{aligned}
$$

Next we need to estimate the photoionization rate. It can be expressed as

$$
P_{3 c}=\frac{\alpha_{p} I}{n \eta}
$$

where $\alpha_{p}$ is the photoionization cross-section. From Griem, ${ }^{3.4}$ the photoionization cross-section at $200 \mathrm{~nm} \alpha_{p}=0.144 \times 10^{-17} \mathrm{~cm}^{2}$. Assuming that the cross-section at $205 \mathrm{~nm}$ is the same

$$
P_{3 c}=\frac{\left(0.144 \times 10^{-17}\right)\left(4 \times 10^{8}\right)}{\left(6.63 \times 10^{-34}\right)\left(1.46 \times 10^{15}\right)}=6.0 \times 10^{8} s^{-1}
$$


We can now write an expression for $N_{3}(t)$. Writing the rate equation $d N_{3} / d t$ gives

$$
\frac{d N_{3}}{d t}=W_{13} N_{1}-\left(A_{31}+A_{32}+Q_{31}+Q_{32}+P_{3 c}\right) N_{3}
$$

Letting $B=A_{31}+A_{32}+Q_{31}+Q_{32}+P_{3 c}$ and $N_{1}^{0}=N_{1}+N_{3}$ we get

$$
\frac{d N_{3}}{d t}=W_{13} N_{1}^{0}-C N_{3}
$$

where $C=B+W_{13}$. Now, since $W_{13} N_{1}^{0}=$ constant, let $z=W_{13} N_{1}^{0}-C N_{3}$, $d z=-C d N_{3}$, we get

$$
-\frac{1}{C} \frac{d z}{z}=d t
$$

Integrating gives

$$
\begin{aligned}
& -\frac{1}{C} \int_{W_{1} N_{1}^{0}}^{W_{1} N_{1}^{0}-C N_{3}\left(\tau_{L}\right)} \frac{d z}{z}=\int_{0}^{\tau_{L}} d t \\
& N_{3}\left(\tau_{L}\right)=\frac{W_{13} N_{1}^{0}\left(1-e^{\left.-C_{\tau_{L}}\right)}\right.}{C} .
\end{aligned}
$$

Now, from BokorA5.8 we find that the 2-photon excitation of state $3 d$ dominates over $3 s$ by at least an order of magnitude for a lineraly polarized laser source. Assuming the laser to be linearly polarized, the allowed spontaneous emission transition that dominates our case is $3 d \rightarrow 2 p$. From Ref. 3.1 this transition rate is

$$
A_{3 d-2 p}=6.465 \times 107 s^{-1}
$$


So, in Eq. 8

$$
\begin{aligned}
C & =A_{3 d-2 p}+Q_{31}+Q_{32}+P_{3 c}+W_{13} \\
& =6.465 \times 10^{7}+1.05 \times 10^{7}+1.95 \times 10^{9}+6.0 \times 10^{8}+8.3 \times 10^{7} \\
C & =2.71 \times 10^{9} s^{-1}
\end{aligned}
$$

For a $5 \mathrm{~ns}$ laser pulse with $N_{1}^{0}=2.3 \times 10^{21} \mathrm{~m}^{-3}$, Eq. 9 gives

$$
\begin{aligned}
& N_{3}(5 n s)=\frac{\left(8.3 \times 10^{7}\right)\left(2.3 \times 10^{21}\right)\left(1-\exp \left[-2.70 \times 10^{9} \times 5 \times 10^{-9}\right]\right)}{2.71 \times 10^{9}} \\
& N_{3}(5 n s)=7.0 \times 10^{19} \mathrm{~m}^{-3} .
\end{aligned}
$$

Note that since $N_{3}\left(\tau_{L}\right)$ is directly proportional to $W_{31}$. Since $W_{31}$ is directly proportional to the laser intensity $I^{2}$, and I is inversely proportional to the focal spot size, by focusing the laser to a smaller spot we could greatly increase $N_{3}\left(\tau_{L}\right)$.

Next, we need to calculate how much of $N_{3}(t)$ will be detected by the CCD camera. The fluorescence signal is given by

$$
F=h v_{e} N_{3} A_{32} \frac{\Omega}{4 \pi} \mid A,
$$

where $v_{\mathrm{e}}$ is frequency of emitted light, $I$ is axial extent of the laser focal spot, and $A$ is the focal area of the laser beam, and $\Omega$ is the collection of solid angle.

$$
\lambda=656 n m \Rightarrow v_{e}=\frac{c}{\lambda}=4.57 \times 10^{14} s^{-1}
$$

The collection solid angle is calculated as follows: 


$$
\Omega=\frac{\pi D^{2}}{4 p^{2}}=\frac{\pi D^{2} M^{2}}{4 f^{2}(M+1)^{2}}=\frac{\pi M^{2}}{4 f_{\#}^{2}(M+1)^{2}} .
$$

Assume that $\mathrm{I}=5 \mathrm{~mm}$. The $\mathrm{CCD}$ camera has a maximum spatial extent of 576 pixels $\times 23 \mu \mathrm{m} /$ pixel $=13 \mathrm{~mm} \Rightarrow M \cong 2.6$. Substituting Eq. 11 into Eq. 10 gives

$$
F=h v_{e} N_{3} A_{32} \mid A \frac{M^{2}}{16 f_{\#}^{2}(1+M)^{2}} \text {. }
$$

Assuming $f_{\#}=4$ optics and $A=10^{-7} \mathrm{~m}^{2}$,

$F=\frac{\left(6.63 \times 10^{-34}\right)\left(4.57 \times 10^{14}\right)\left(7.0 \times 10^{19}\right)\left(6.645 \times 10^{7}\right)(2.6)^{2}(.005)\left(1 \times 10^{-7}\right)}{16\left(4^{2}\right)(2.6+1)^{2}}$

$$
F=1.4 m W
$$

This fluorescence signal $F$ is the signal incident on the photocathode of the intensifier. Next we need to include the gain of the intensifier. Including the photocathode sensitivity at $\sim 600 \mathrm{~nm}$ and the phosphor output sensitivity, the overall gain of a typical intensifier can be expressed as: $\mathrm{A} 5.10$

$$
\text { GR }=2600 \text { (green power) out } / \text { (red power) in } .
$$

The total signal flux incident on the CCD array can then be calculated as follows:

$$
\begin{aligned}
P_{T} & =\frac{\dot{E}_{\text {out, intensifier }}}{(h v)_{\text {out }}} \\
& =\frac{\dot{E}_{\text {in, intensifier }}}{(h v)_{\text {out }}} G_{R}
\end{aligned}
$$




$$
\begin{aligned}
& =\frac{F}{(h v)_{\text {out }}} G_{R}=\frac{F \lambda}{h c} G_{R} \\
P_{T} & =\frac{\left(1.4 \times 10^{-3} \mathrm{~W}\right)\left(550 \times 10^{-9} \mathrm{~m}\right)}{\left(6.63 \times 10^{-34} \mathrm{~J} \cdot \mathrm{s}\right)\left(3 \times 10^{8} \mathrm{~m} / \mathrm{s}\right)}(2600) \\
P_{T} & =1.0 \times 10^{19} \text { photons } / \mathrm{s}
\end{aligned}
$$

Now, we can calculate the mean photon flux per pixel

$$
\begin{aligned}
& P_{p}=\frac{P_{T}}{\text { no. of pixels }}=\frac{1.0 \times 10^{19}}{384 \times 576} \\
& P_{p}=4.7 \times 10^{13} \text { photons } / \text { pixel s. }
\end{aligned}
$$

Finally, we can calculate the signal-to-noise (SNR) ratio as follows:A5.11

$$
S N R=\frac{P_{p} t_{1} Q_{e}}{\sqrt{\left(P_{p}+B\right) t_{1} Q_{e}+D t_{2}+N_{\tau}^{2}}},
$$

where $t_{1}$ is the duration of fluorescence signal, $Q_{e}$ is the quantum efficiency of CCD, $B$ is the background photon flux on CCD per pixel (photons $/ s$ ), $D$ is the dark current, $t_{2}$ is the integration time of the CCD, and $N_{\tau}$ is the readout noise of the CCD.

Assuming the following values for the above variables:

$$
\begin{aligned}
& t_{1}=5 \mathrm{~ns} \\
& Q_{e}=0.30 \text { at } 55 \mathrm{~nm} \\
& B=\text { negligible } \\
& t_{2}=2.2 \mathrm{~s} \quad(100 \mathrm{k} \text { pixel } / \mathrm{s} \text { readout rate }) \\
& D=12 \text { electrons } / \mathrm{s} \text { pixel } \\
& N_{\tau}=25 \text { electrons } / \text { pixel }
\end{aligned}
$$

$$
S N R=260
$$


Because conservative estimates for the variables in these calculations were used this SNR indicates that a 2-photon excitation of the hydrogen plasma armature produced by the railgun should be possible. 


\section{BIBLIOGRA PHY}

1.1 Marion, J. B., and Hornyak, W.F.; Physics: For Science and Engineering, Saunders College Publishing, Philadelphia, 1982.

1.2 Chen, Y. K. and Howell, J. R., "Two-Dimensional Effects of Plasma VaporShield Radiation on Fusion Reactor First-Wall Conditions," Heat-TransferNiagara Falls, AICHE Symposium Series, Vol. 80, no 236, pp 410.415, August 1984.

1.3 Deluca, R.J., "Characterization of Metallic Coatings and Thin Films Produced by Railgun Deposition," M.S. Thesis, The Department of Mechanical Engineering, The University of Texas, Austin, May 1987.

1.4 Spann, M. L., Brown, L. D., "Formation of Amorphous Metal by Hypervelocity Impact," EL-3238, Research Project 2115-6, Final Report, The University of Texas, Austin, Oct 1983.

1.5 Thompson, W.B.: An Introduction to Plasma Physics, Pergamon Press, Oxford, 1962.

1.6 Marshall, J., "Performance of a Hydromagnetic Plasma Gun," Physics of Fluids, vol 3, p 134, 1960. 
1.7 Aigner, S., Igenbergs, E., "Friction and Ablation Measurements in a Round Bore Railgun," Fourth Symposium on Electromagnetic Launch Technology, Austin, Texas, April, 1988.

1.8 Parker, J. V., "The SRS Railgun: A New Approach to Restrike Control," IEEE Transactions on Magnetics, Vol. Mag-20, No. 2, March 1984.

1.9 Holland, L. D., "Distributed Energy Store Railguns Experiment ard Analysis," PhD Dissertation, The University of Texas, Austin, May, 1984.

1.10 Derbidge, T. C., Powars, C., "Plasma Armature Heat Transfer and Rail Ablation Diagnostics," IEEE Transactions on Magnetics, Vol 25, No. 1, Jan 1989.

1.11 Jamison, K. A., Burden, H. S., "Measurements of Plasma Properties from a Large Bore, Plasma Armature Railgun," IEEE Transactions on Magnetics, Vol. Mag-20, No. 2, March 1984.

1.12 Deluca, R. J., "Characterization of Metallic Coatings and Thin Films Produces by Railgun Deposition," M. S. Thesis, The University of Texas at Austin, May 1987.

1.13 Thornhill, L. D. and Batteh, J. H., "Armature Options for Hypervelocity Railguns," IEEE Transactions on Magnetics., vol. 25, no. 1, pp. 552$557,1989$.

1.14 Ray, P. K., "Characterization of Plasma in a Railgun," IEE Proceedings, vol. 133, Pt A. no. 1, pp. 38-43, 1986. 
1.15 Marshall, R. A., "Structure of Plasma Armature of a Railgun," IEEE Transactions on Magnetics, vol. MAG-22, no. 6, pp. 1609-1612, 1986.

1.16 Marshall, R. A., "Plasma Puffing from a Railgun Armature," IEEE Transactions on Magnetics, vol. Mag-20, no. 2, pp. 264-267, 1984.

1.17 Sedghinasab, A., Keefer, D. R., and Crowder, H. L. "In-Bore Spectral Measurements in a Plasma-Armature Railgun," IEEE Transactions Plasma Science, vol. 17, no. 3, pp. 360-364, 1989.

1.18 Kiwamoto, Y., "Small barium rail gun for plasma injection," Review of Scientific Instrumentation, vol. 51, no. 3, pp. 285-287, 1980.

2.1 Petersen, D. R., Weeks, D. A., Zowarka, Jr., R. C., Cook, R. W., Weldon, W. F., "Testing of a High Performance, Precision-Bore Railgun," IEEE Transactions on Magnetics, Vol MAG-22, no 6, p. 1662-1668, November, 1986.

2.2 Cook, R. W., "Observation and Analysis of Current Carrying Plasmas in a Railgun," Master's Thesis, The University of Texas at Austin, December, 1985.

2.3 Torre, O. C., "Measurements on High Temperature Gases in an Electromagnetic Shock Tube," Master's Thesis, The University of Texas at Austin, August, 1987.

2.4 Burkhardt, L. C., Loveberg, R. H., "Current Sheet in a Coaxial Plasma Gun," Physics of Fluids, Vol. 5, No. 3, March 1962. 
3.1 (a) L. Wiese, M.W. Smith, and B.M. Glennon, "Atomic Transition Probabilities, Vol I: Hydrogen Through Neon," W. , NSRDS-NBS-4, U.S. Government Printing Press, Washington DC, May 20, 1966.

(b) L. Wiese, M.W. Smith, and B.M. Glennon, "Atomic transition Probabilities, Vol II: Sodium through Calcium," NSRDS-NBS-22, October 1969.

(c) C. E. Moore, "Selected Tables of Atomic Spectra, Atomic Energy Levels and Multiplet Tables, Si II, Si III, Si IV," NSRDS-NBS 3, Section 1, June 25, 1965.

(d) C. E. Moore,"Selected Tables of Atomic Spectra, Atomic Energy Levels and Multiplet Tables, N I, N II, N III, " NSRDS-NBS 3, Section 5, May 1975.

3.2 F. Mastrup, W. L Wiese, "Experimentelle Bestimmung der Oszillatorenstarken Einger," Z. Astrophys., vol. 44, no. 3, pp. 259-279, 1958.

3.3 R. H. Garstang, "Intermediate Coupling Li ve Strengths," Mont. Not. R. Astron. Soc. vol. 114, no. 1, pp 118-133, 1954.

3.4 H. R. Griem, Plasma Spectroscopy, McGraw Hill, New York, NY, 1964.

3.5 C. W. Allen, Astrophysical Quantities, The Athelone Press, University of London, London, 1971. 
3.6 C. R. Vidal, J. Cooper, and E. W. Smith, "Hydrogen Stark-Broadening Tables," Astrophysical Journal Supplemental Series No. 214, 25:37-136, 1973.

3.7 Bengtson, R.D., Personal communication, Department of Physics, The University of Texas at Austin, April, 1991.

3.8 V. Bakshi and R. J. Kearney, "An investigation of local thermodynamic equilibrium in an argon plasma jet at atmospheric pressure," Journal of. Quantitative Spectroscopic Radiation Transfer, vol. 41, no. 2, pp. 369$376,1989$.

3.9 P. R. Bevington, Data Reduction and Error Analysis for the Physical Sciences, McGraw-Hill Book Company, New York, NY, 1969.

3.10 J. Oxenius, Kinetic Theory of Particle and Photons, Springer Series in Electrophysics 20, Springer-Verlag, Berlin, Heidelberg, 1986.

4.1 Bengtson, R. D., Personal communication, Department of Physics, The University of Texas at Austin, April, 1991.

5.1 Chen, Y. K., Varghese, P. L., and Howell, J. R., "Interaction between a high kinetic energy plasma jet and a target surface," Internal report, Center for Fusion Engineering, The University of Texas at Austin, December, 1986. 
5.2 Talmi, Yair, Personal communication, Princeton Instruments Inc., Princeton, New Jersey, 1989.

5.2 Manuals, Princeton Instruments Inc., Princeton, New Jersey, 1990.

5.3 Beer, F. P. and Johnston, Jr., E., R., Mechanics of Materials, McGraw-Hill Book Company, New York, New York, 1981.

A5.1 Vincenti, W. G. and Kruger, C. H., Jr., Introduction to Physical Gas Dynamics, Wiley, New York 1967.

A5.2 Bakshi, V., Propp, A. D., Varghese, P. L., and Howell, J. R., "Diagnostics of Railgun Plasma Armatures," Submitted for Publication to IEEE Transactions on Plasma Science, September 1, 1990.

A5.3 McDaniel, E. W., Atomic Collisions, Electron and Photon Projectiles, Wiley, New York, 1989.

A5.4 Calloway, Journal of Physics Reports, Vol. 45, No. 2, pp. 89-173, 1972.

A5.5 McCrea, D. and McKirgan, T. V. M., Proceedings of the Physics Society of London, Vol. A, No. 75, pp. 235-242, 1960.

A5.6 Boyd, T. J. M.,Proceedings of the Physics Society of London, Vol. A, No. 72, pp. 523-531, 1958.

A5.7 Eckbreth, A. C., Laser Diagnostics for Combustion Temperature and Species, Abacus Press, Cambridge, MA, 1988. 
A5.8 Bokor, J., Freeman, R. R., White, J. C., and Storz, R. H., Physics Review A, Vol. 24, No. 1, pp. 612-614, 1981.

A5.9 Bischel, W. K., Bryce, E. P., and Crosley, D. R., Applied Optics, Vol. 21, No. 8, pp. 1419-1429, 1982.

A5.10 Flynt, W. E., "Derivation of Image Intensifier Gain in Terms of Watts or Photons," Memorandum, Varo Inc., Electron Devices Division, Garland, TX, 1990.

A5.11 Schemp, W. V., and Toker, E., "Signal-to-Noise Ratio Calculations," Application Note No. 1, Photometrics Ltd., Tuscon, AZ, 1990.

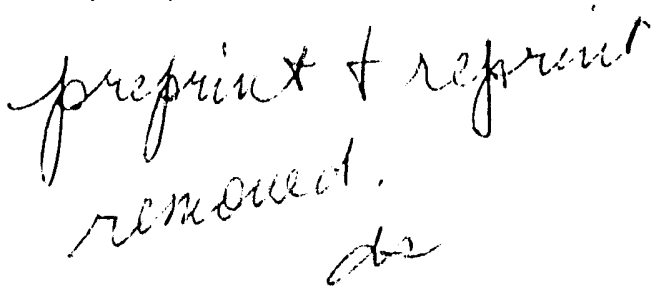



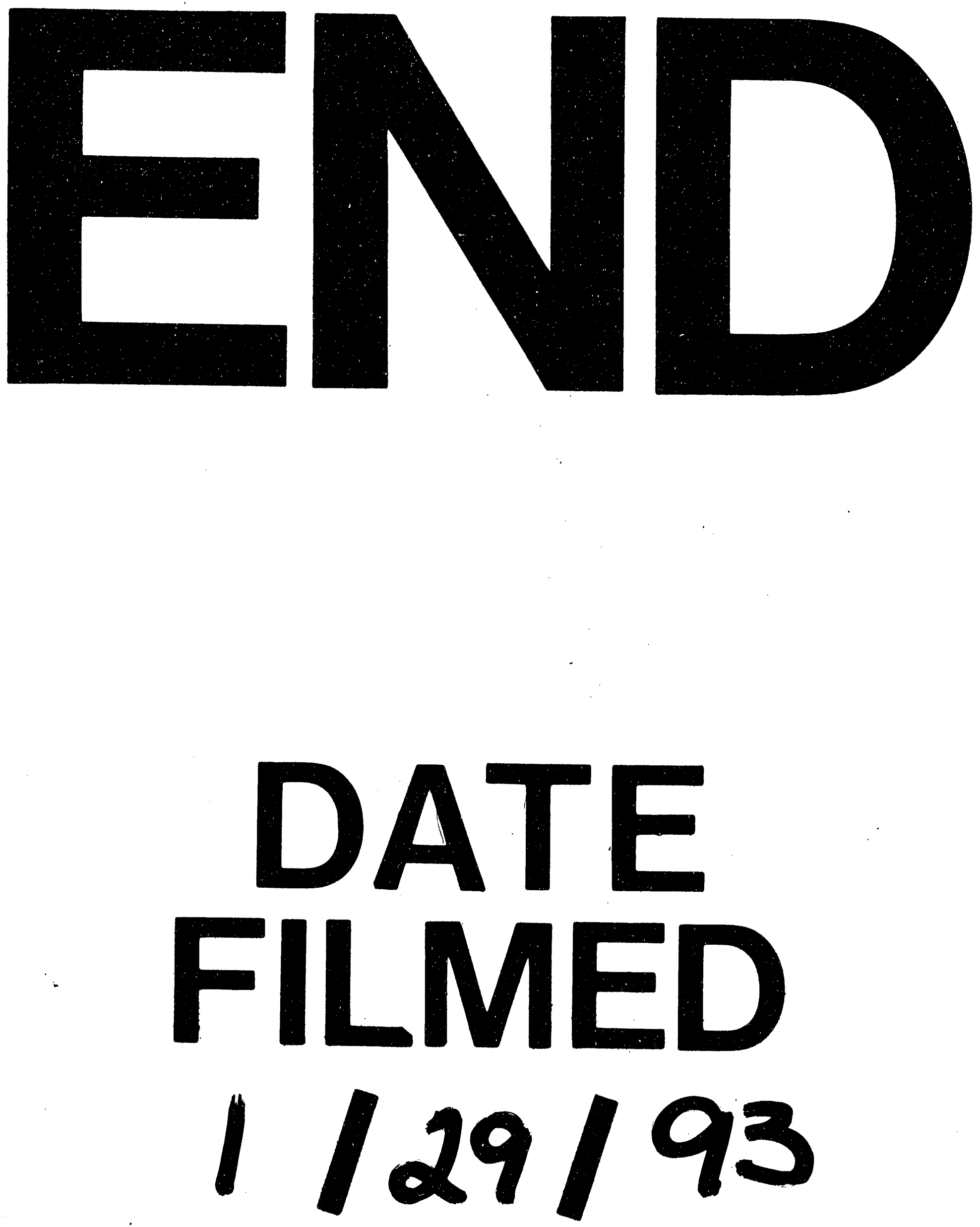
